\title{
Estratificação de Risco para Eventos Cardíacos Maiores em Pacientes Submetidos ao Implante de Stents Farmacológicos. Escore DESIRE
}

\author{
Tese apresentada ao Instituto Dante Pazzanese de Cardiologia, \\ Entidade Associada da Universidade de São Paulo \\ para a obtenção do título de Doutor em Ciências.
}

Área de Concentração: Medicina, Tecnologia e Intervenção em Cardiologia.

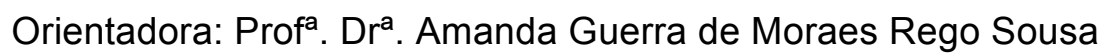

Versão corrigida. Resolução CoPGr 6018/11, de 01 novembro de 2011. A versão original está disponível na Biblioteca do IDPC.

São Paulo 
Dados Internacionais de Catalogação na Publicação (CIP)

Preparada pela Biblioteca do Instituto Dante Pazzanese de Cardiologia

Creprodução autorizada pelo autor

\section{Moreira, Adriana Costa}

Estratificação de risco para eventos cardíacos maiores em pacientes submetidos ao implante de stents farmacológicos: escore DESIRE / Adriana Costa Moreira

Tese(doutorado)--Instituto Dante Pazzanese de Cardiologia Universidade de São Paulo

Área de Concentração: Medicina, Tecnologia e Intervenção em

Cardiologia

Orientador: Profa ${ }^{\text {D }} r^{\mathrm{a}}$. Amanda Guerra Moraes Rego Sousa

Descritores: 1. Coronariopatia. 2. Intervenção Coronária Percutânea. 3. Stents Farmacológicos. 4. Fatores de Risco. 5. Prognóstico.

USP/IDPC/Biblioteca/072/17 
Dedicatória 
Às minhas queridas avós Alzira e Rita, que souberam superar com muita sabedoría os momentos maís difíceís e construír com doçura, força e dedicação a nossa familía.

Aos meus país, Wenceslau e Lúcía, pelo exemplo de vida e pelo apoío incansável na busca pelos meus sonhos.

Ao meu irmão Chrístiano, pela amizade presente e o apoio constante.

Ao meu esposo Breno por estar sempre presente, pelo apoí irrestrito, incentivo constante e por me fazer enfrentar os desafios com otimismo e confíança

Aos meus amados filhos, Arthur e Isabella, razão de tudo, a quem dedico cada conquista e os meus mais bonitos sonhos 
Agradecimentos 
'̇̀ Profa. Dra. Amanda Guerra de Moraes Rego Sousa, cuja conduta exemplar e retídão têm sido inspiração desde o inicio da minha formação. Minha maís sincera gratidão pela inestimável orientação nesta tese.

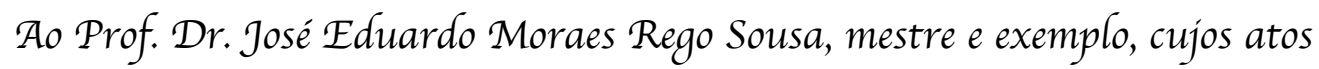
fundamentam a minha prática médíca. Agradeço intensamente por guiar meu desenvolvimento profissional.

Ao grupo de pesquisadores do Registro $\mathcal{D E S T R E : ~} \mathcal{D r}$ José de Ríbamar Costa Ir, Dr Ricardo Costa, Eng Cantidío Campos, Sra Luciana Lopes e Sra Juliana Ramalho companheiros nesta desafiadora jornada.

Ao estatístico Lucas Petri pelo dedicado e criterioso trabalho na execução desta tese.

À Superintendência e às Diretorias do Instítuto de Ensino e Pesquísa do Hospital do Coração - ASS e da Associação do Sanatório Sirio - ASS, por todo o apoío e por zelarem pela contínua evolução do Registro $\mathcal{D} \mathcal{E S} \mathcal{R} \mathcal{E}$. 
Aos mestres e amigos do Serviço de Cardíología Intervencionista do Hospital do Coração - ASS: Dr Valmír Fontes, Dr. Galo Maldonado, Dr Manuel Cano, Dr. J. Ríbamar Costa Jr, Dr. Carlos Pedra, Dr Antônio Kambara, Dr Fausto Feres, $\mathcal{D}$ r Sergio Braga, Dr Rodolfo Staico e Dr Dimytri Siqueira pela convivência harmoniosa na prática díaría e cooperação essencial para a realização deste estudo.

Às enfermeiras, aos técnicos (as) de enfermagem e funcionários do Setor de Cardiologia Intervencionista do Hospital do Coração - ASS, que, com profunda dedicação, tornam possivel nossa prática diáría.

Ao Sr Wagner Vieira pinto, pela valiosa contríbuição à realização dessa tese.

Ȧ Sra Luciana Lopes, pela colaboração intensa na execução dessa tese.

Aos pacientes, que de forma generosa e altruísta, consentiram participar deste estudo. 
"Procurar conhecimento é muitas vezes aprender a duvidar" Antoinette Deshoulières 
Normalização adotada

Esta tese está de acordo com as seguintes normas, em vigor no momento desta publicação:

Referências: adaptado de International Committee of Medical Journals Editors (Vancouver).

Universidade de São Paulo. Faculdade de Medicina. Divisão de Biblioteca e Documentação. Guia de apresentação de dissertações, teses e monografias. Elaborado por Anneliese Carneiro da Cunha, Maria Julia de A. L. Freddi, Maria F. Crestana, Marinalva de Souza Aragão, Suely Campos Cardoso, Valéria Vilhena. 3a ed. São Paulo: Divisão de Biblioteca e Documentação; 2011.

Abreviaturas dos títulos dos periódicos de acordo com List of Journals Indexed in Index Medicus. 


\title{
Sumário
}

\author{
Lista de abreviaturas \\ Lista de símbolos \\ Lista de tabelas \\ Lista de figuras \\ Resumo \\ Summary
}

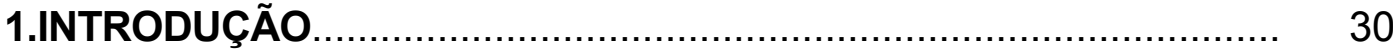

1.1 Evoluções da intervenção coronária percutânea......................... 34

1.2 Stents farmacológicos: estudos fundamentais e registros clínicos........................................................................... 38

1.3 Importância atual dos escores de risco......................................... 41

1.4 Os escores de risco para revascularização miocárdica..................... 42

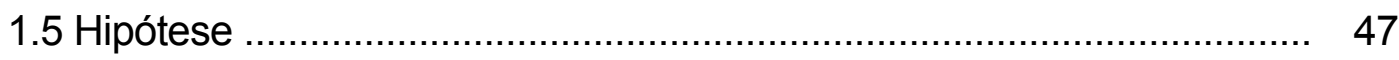

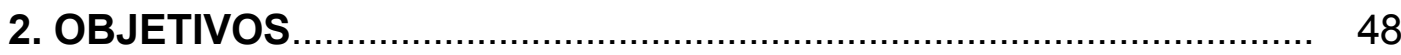

2.1 Objetivos primários................................................................ 49

2.2 Objetivos secundários........................................................ 49

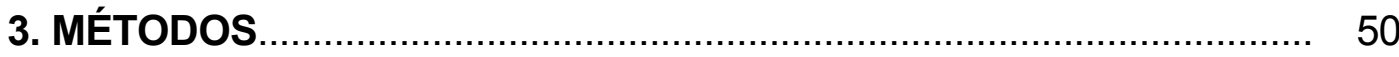

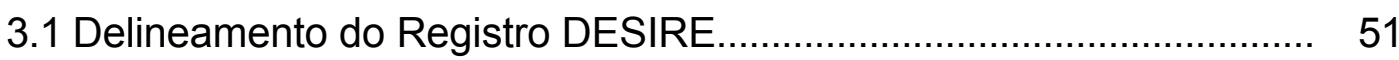

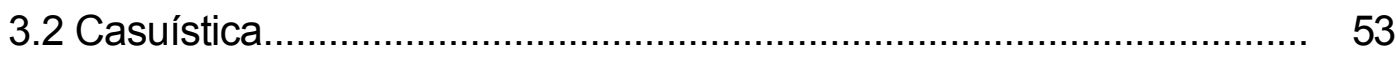

3.2.1 Critérios de inclusão............................................................ 53

3.2.2 Critérios de exclusão........................................................ 54

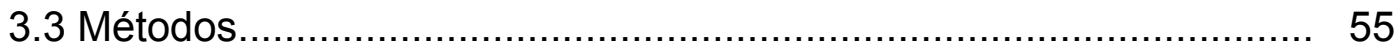


3.3.1 Preparo do paciente, aspectos técnicos do procedimento e protocolo medicamentoso adjunto. 55

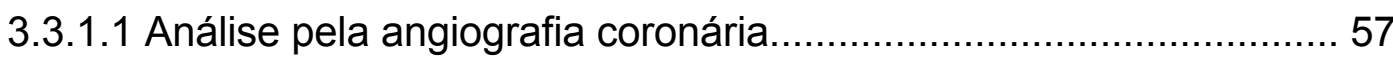

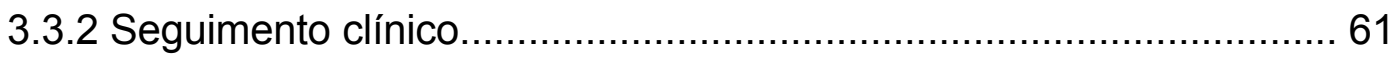

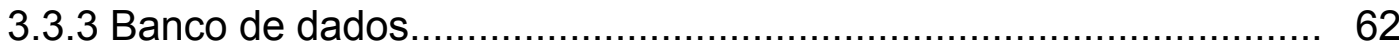

3.3.3.1 Variáveis analisadas para o desenvolvimento do escore DESIRE e suas definições......................................... 64

3.3.4 Definições dos desfechos............................................................ 70

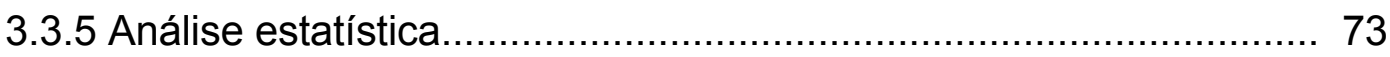

3.3.5.1 Análise descritiva............................................................... 74

3.3.5.2 Desenvolvimento do escore DESIRE..................................... 74

3.3.5.3 Validação do escore DESIRE............................................... 76

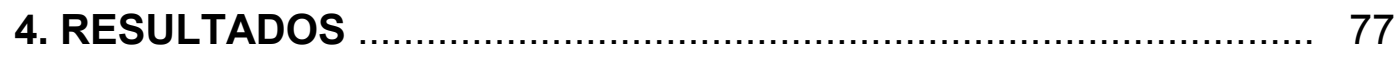

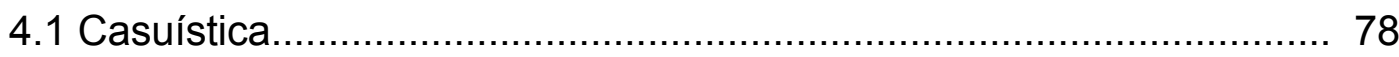

4.2 Características clínicas............................................................... 79

4.3 Características angiográficas e aspectos técnicos............................ 81

4.3.1 Dados angiográficos pré-ICP.................................................... 81

4.3.2 Aspectos técnicos................................................................. 85

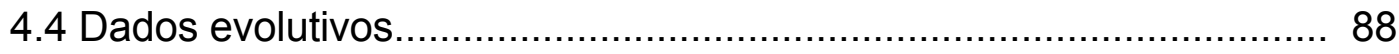

4.4.1 Eventos cardíacos maiores......................................................... 89

4.4.2 Trombose protética.................................................................. 91

4.5 Desenvolvimento e validação do

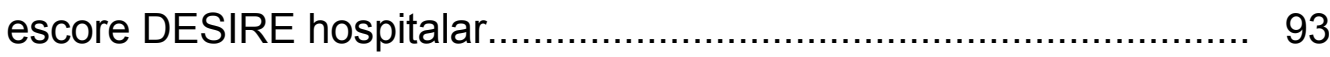

4.5.1 Desenvolvimento do escore DESIRE hospitalar........................... 93 
4.5.2 Validação do escore DESIRE Hospitalar

4.6 Desenvolvimento e validação do

escore DESIRE tardio

4.6.1 Desenvolvimento do escore DESIRE tardio.............................. 109

4.6.2 Validação do escore DESIRE tardio........................................ 123

4.7 Resumo prático do escore DESIRE......................................... 125

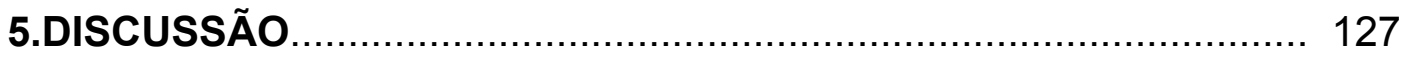

5.1 O Registro DESIRE ........................................................... 129

5.2 O escore DESIRE ............................................................. 134

5.3 Considerações sobre a acurácia do escore DESIRE .................... 140

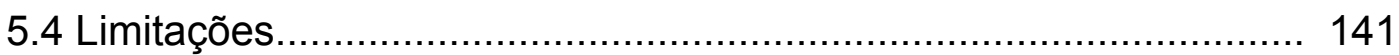

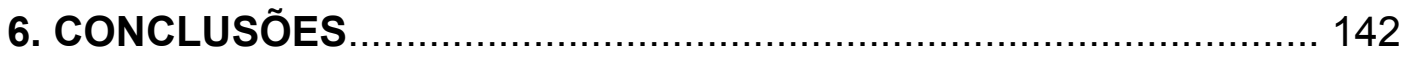

7. REFERÊNCIAS BIBLIOGRÁFICAS ............................................ 145 
Listas 


\section{LISTA DE ABREVIATURAS}

$\begin{array}{ll}\text { ACC } & \text { American College of Cardiology } \\ \text { ACQ } & \text { angiografia coronária quantitativa } \\ \text { ADP } & \text { adenosina difosfato } \\ \text { AHA } & \text { American Heart Association } \\ \text { AM } & \text { Adriana Moreira } \\ \text { ARC } & \text { Academy Research Consortium } \\ \text { AS } & \text { Amanda Sousa } \\ \text { ATC } & \text { angioplastia transluminal coronária } \\ \text { AVE } & \text { acidente vascular encefálico } \\ \text { BARI } & \text { Bypass Angioplasty Revascularization Investigation } \\ \text { CAAE } & \text { Certificado de Apresentação para Apreciação Ética } \\ \text { CASS } & \text { Coronary Artery Surgery Study } \\ \text { CD-R } & \text { Compact Disc Recordable } \\ \text { CENIC } & \text { Central Nacional de Intervenções Cardiovasculares } \\ \text { CICr } & \text { clearance de creatinina } \\ \text { CO } 2 & \text { gás carbônico } \\ \text { Cr } & \text { creatinina } \\ \text { CRM } & \text { cirurgia de revascularização miocárdica } \\ \text { CX } & \text { artéria circunflexa } \\ \text { DA } & \text { artéria descendente anterior } \\ \text { DAC } & \text { doença arterial coronária } \\ & \end{array}$




\begin{tabular}{ll} 
DCV & doença cardiovascular \\
DESIRE & Drug Eluting Stent In the Real World \\
DICOM & Digital Imaging and Communications in Medicine \\
DIV & diâmetro interpolado do vaso \\
DM & diabete melito \\
DML & diâmetro mínimo do lúmen \\
DP & desvio-padrão \\
DTA & dupla terapêutica antiplaquetária \\
ECM & eventos cardíacos maiores \\
ECG & eletrocardiograma \\
ECR & estudos clínicos randomizados \\
ED & grau de obstrução \\
EUA & Estados Unidos da América \\
FE & fração de ejeção \\
FVE & função ventricular esquerda \\
FIM & first in man \\
GI & ganho imediato \\
GL & ganho líquido \\
H $2 O$ & água \\
HAS & hipertensão arterial sistêmica \\
HCor-ASS & Hospital do Coração - Associação do Sanatório Sírio \\
HR & hazard ratio, taxa de risco \\
IC & intervalo de confiança \\
ICC & insuficiência cardíaca congestiva \\
\hline DE &
\end{tabular}




$\begin{array}{ll}\text { ICP* } & \text { intervenção coronária percutânea } \\ & \text { * será aplicada a mesma nomenclatura para o singular e o plural } \\ \text { IM } & \text { infarto do miocárdio } \\ \text { IMC } & \text { índice de massa corpórea } \\ \text { JES } & \text { José Eduardo Sousa } \\ \text { JRCJ } & \text { José Ribamar Costa Junior } \\ \text { L } & \text { lesão } \\ \text { MEPS } & \text { Medical Expenditure Panel Survey } \\ \text { N } & \text { número de casos } \\ \text { NCDR } & \text { National Cardiovascular Data Registry } \\ \text { NHLBI } & \text { National Heart, Lung and Blood Institute } \\ \text { NYHA } & \text { New York Heart Association } \\ \text { OR } & \text { odds ratio, razão de chances } \\ \text { P } & \text { paciente } \\ \text { PLA } & \text { ácido poliláctico } \\ \text { PLGA } & \text { poli(ácido glicólico) poli-lactídeo-co-glicolídio } \\ \text { PT } & \text { perda tardia } \\ \text { RC } & \text { Ricardo Costa } \\ \text { RLA } & \text { revascularização da lesão-alvo } \\ \text { ROC } & \text { receiver operator characteristic curve } \\ \text { SCA } & \text { síndrome coronária aguda } \\ \text { SF* } & \text { stents farmacológicos } \\ \text { TCE } & \text { será aplicada a mesma nomenclatura para o singular e o plural } \\ & \end{array}$


TIMI

Thrombolysis in Myocardial Infarction

V

vaso

VE

ventrículo esquerdo 


\section{LISTA DE SÍMBOLOS}

$\begin{array}{ll}= & \text { igual } \\ \geq & \text { maior ou igual a } \\ \pm & \text { mais ou menos } \\ > & \text { maior que } \\ { }^{\circ} & \text { marca registrada } \\ \leq & \text { menor ou igual a } \\ < & \text { menor que } \\ \mathrm{mg} / \mathrm{dl} & \text { miligrama por decilitro } \\ \mathrm{mm} & \text { milímetro } \\ \% & \text { porcentagem } \\ \mathrm{kg} & \text { quilograma } \\ \mathrm{Ul} & \text { unidades internacionais }\end{array}$




\section{LISTA DE TABELAS}

Tabela 1- Classificação das lesões-alvo baseada nas características angiográficas de acordo com o ACC/AHA 44

Tabela 2- $\quad$ Características clínicas dos pacientes das coortes B, B1 e B2 ......................................

Tabela 3- $\quad$ Apresentação clínica das coortes B, B1 e B2 .81

Tabela 4- Características angiográficas dos pacientes das coortes B, B1 e B2

Tabela 5- Características angiográficas pré-procedimento dos vasos-alvo das coortes B, B1 e B2

Tabela 6- Características angiográficas pré-procedimento das lesões-alvo das coortes B, B1 e B2

Tabela 7- Dados da angiografia coronária quantitativa pré-ICP das coortes $B, B 1$ e B2 84

Tabela 8- $\quad$ Características dos procedimentos das coortes B, B1 e B2 .86

Tabela 9- Dados da angiografia coronária Quantitativa(ACQ) pós-ICP das coortes B, B1 e B2

Tabela 10- Distribuição dos eventos cardíacos maiores nas coortes B, B1 e B2 na fase hospitalar .90 
Tabela 11- Distribuição dos eventos cardíacos maiores das coortes B, B1 e B2 na fase tardia.

Tabela 12- Incidência acumulada de trombose protética nas coortes B, B1 e B2 91

Tabela 13- Classificação e distribuição temporal das 34 tromboses protéticas nas coortes B, B1 e B2, até 2 anos de seguimento, de acordo com Academic Research Consortium (ARC). .92

Tabela 14- Resultados da análise da associação da idade, do gênero e dos fatores de risco, com os ECM hospitalares pela regressão logística univariada.....94

Tabela 15- Resultados da análise da associação dos eventos e procedimentos cardiovasculares prévios com os ECM hospitalares pela regressão logística univariada. .95

Tabela 16- Resultados da análise da associação das apresentações clínicas com os ECM hospitalares pela regressão logística univariada .96

Tabela 17- Resultados da análise da associação da extensão da DAC e Função ventricular esquerda com os ECM hospitalares pela regressão logística univariada......96

Tabela 18- Resultados da análise da associação das variáveis angiográficas (vasos-alvo) com os ECM hospitalares pela regressão logística univariada. 
Tabela 19- Resultados da análise da associação das variáveis angiográficas (lesões-alvo)com os ECM hospitalares pela regressão logística univariada......................98

Tabela 20- Resultados da análise da associação das variáveis angiográficas com os ECM hospitalares pela regressão logística univariada

Tabela 21- Variáveis do modelo de regressão logística múltipla para o escore DESIRE hospitalar 100

Tabela 22- Modelo final do escore DESIRE hospitalar 101

Tabela 23- Faixas de risco para ECM categorizadas pelo escore DESIRE hospitalar 105

Tabela 24- Faixas de risco para ECM categorizadas pelo Escore DESIRE tardio (Pontuação). 105

Tabela 25- Distribuição dos pacientes da coorte B1 de acordo com as três faixas de risco (probabilidade logística) e pontuação obtida 106

Tabela 26- Resultados da análise da associação da idade, gênero e dos fatores de risco com os ECM evolutivos pela regressão de Cox 
Tabela 27- Resultados da análise da associação dos eventos e procedimentos cardiovasculares prévios com os ECM evolutivos pela regressão de Cox

Tabela 28- Resultados da análise da associação das apresentações clínicas com os ECM evolutivos pela regressão de Cox

Tabela 29- Resultados da análise da associação da extensão da DAC e Função ventricular esquerda com os ECM evolutivos pela regressão de Cox

Tabela 30- Resultados da análise da associação das variáveis angiográficas (vasos-alvo) com os ECM evolutivos pela regressão de Cox

Tabela 31- Resultados da análise da associação das variáveis angiográficas (lesões-alvo)com os ECM evolutivos pela regressão de Cox

Tabela 32- Resultados da análise da associação das variáveis angiográficas com os ECM evolutivos pela regressão de Cox 115

Tabela 33- Variáveis do modelo inicial de regressão de Cox para o escore DESIRE tardio

Tabela 34- Modelo final do escore DESIRE tardio 117 
Tabela 35- Faixas de risco para ECM categorizadas pelo escore DESIRE tardio

Tabela 36- Faixas de risco para ECM categorizadas pelo

Escore DESIRE tardio (Pontuação).

Tabela 37- Distribuição dos pacientes da coorte B1 de de acordo com as três faixas de risco definidas pelo escore DESIRE tardio

Tabela 38- Registros clínicos utilizados para o desenvolvimento de escores de risco para estimativas de ECM após ICP na última década 133 


\section{LISTA DE FIGURAS}

Figura 1- Taxas de doenças cardiovasculares na população

brasileira de acordo com os grupos etários 31

Figura 2- $\quad$ Análise de regressão linear simples e comparação das linhas de regressão entre homens e mulheres, da mortalidade por doença arterial coronária (DAC) para os períodos de 1980 a 2006 e de 2007 a 2012 .

Figura 3- $\quad$ Custos diretos e indiretos para DCV nos EUA entre 2011 e 2012.

Figura 4- Evolução comparativa das formas de revascularização miocárdica (ICP e CRM) ao longo dos anos no Hospital do Coração da Associação do Sanatório Sírio (HCor-ASS)

Figura 5- Inclusão consecutiva dos 7.074 pacientes tratados com 12.497 SF a partir de maio/2002 até dezembro/2016, no Registro DESIRE

Figura 6- Sobrevida dos pacientes mantidos em tratamento medicamentoso no Estudo CASS.

Figura 7- Inclusão consecutiva dos pacientes tratados com SF a partir de 2002, no Registro DESIRE. 52

Figura 8- Fluxograma de inclusão de pacientes no

Registro DESIRE. 78 
Figura 9- Relação entre os modelos logístico e simplificado do escore DESIRE hospitalar. 102

Figura 10- Acurácia dos modelos logísitico e simplificado do escore DESIRE hospitalar 103

Figura 11- Probabilidades estimadas pelo modelo para cada valor do escore DESIRE hospitalar simplificado 104

Figura 12- Esquema do escore DESIRE para cálculo da probabilidade de ECM hospitalares. 107

Figura 13 Validação do escore DESIRE hospitalar. 108

Figura 14- Acurácia dos modelos logísticos e simplificado do escore DESIRE hospitalar na coorte B2 (validação) .... 109

Figura 15- Relação entre os modelos logístico e simplificado do escore DESIRE tardio

Figura 16- Acurácia do escore DESIRE tardio 119

Figura 17- Probabilidades estimadas pelo modelo para cada valor do escore DESIRE tardio simplificado 120

Figura 18- Esquema do escore DESIRE para cálculo da probabilidade de ECM tardios 123

Figura 19- Validação do escore DESIRE tardio. 124

Figura 20- Acurácia do escore DESIRE tardio na coorte B2........ 125

Figura 21- Resumo prático do escore DESIRE para as fases hospitalar e tardia

Figura 22- Registro DESIRE: preditores independentes de ECM pós-SF nas fases hospitalar e tardia pós-alta. 136 
RESUMO

Moreira, AC. Estratificação de Risco para Eventos Cardíacos Maiores em Pacientes Submetidos ao Implante de Stents Farmacológicos. Escore DESIRE[tese]. São Paulo: Instituto Dante Pazzanese de Cardiologia, Universidade de São Paulo; 2017. 166p.

INTRODUÇÃO: A estratificação de risco, pré intervenção coronária percutânea, é um elemento-chave para auxiliar na tomada de decisão oferecendo o nível de expectativas de resultados imediatos e tardios. Esta investigação tem como objetivo desenvolver o escore DESIRE para predizer o risco de ocorrência de eventos cardíacos maiores(ECM) em pacientes $(P)$ submetidos ao implante de stents farmacológicos(SF) na prática clínica diária. MÉTODOS: Desde 2002 todos os pacientes consecutivos, tratados com SF , foram incluídos no Registro DESIRE (Drug-Eluting Stents In REal world), não-randomizado. Desenvolvemos o escore DESIRE, a partir da análise retrospectiva dos dados dos $\mathrm{P}$ incluídos entre janeiro de 2007 e dezembro de 2012 uma vez que esta população representava melhor a prática contemporânea da cardiologia intervencionista. As associações das variáveis com os eventos foram testadas pelos testes chi-quadrado e t de Student. Devido às diferenças entre as fases analisadas, decidimos por dois modelos de predição de riscos: hospitalar (Regressão logística) e tardio (Cox) estimando o tempo até o primeiro evento. Após o desenvolvimento, o escore foi aplicado prospectivamente nos pacientes incluídos entre janeiro de 2013 e dezembro de 2014. As estimativas de risco obtidas foram comparadas às taxas de ECM observadas, validando o escore DESIRE nas duas fases. RESULTADOS: 4.061 pacientes compõem a população do estudo sendo que destes, 2.863 P constituem a coorte B1 que foi utilizada para o desenvolvimento do escore e 1.198 $\mathrm{P}$ na coorte $\mathrm{B} 2$ na qual o escore foi aplicado e validado. As variáveis e seus respectivos pontos para o escore DESIRE hospitalar que variou de 0 a 37 pontos foram: idade em anos $(\leq 49=$ $0 ; 50$ a $59=1 ; 60$ a $69=2 ; 70$ a $79=4 ; \geq 80=6$ ); cirurgia de revascularização miocárdica prévia(1); doença vascular periférica (5); insuficiência renal (3); síndrome coronária aguda (3); doença multiarterial (3); ponte de safena (6); lesão com trombo (5) e lesão longa (5). Compuseram o escore DESIRE tardio ( 0 a 45 pontos) as seguintes variáveis: diabete melito com medicação oral (4) ou com insulina (9); cirurgia de revascularização miocárdica prévia (2); doença vascular periférica (6); síndrome coronária aguda (5); doença multiarterial (4); FE<40\% (6); ponte de safena (8) e vaso de pequeno calibre (5). Definimos 3 faixas de risco 
para cada um dos escores categorizando os pacientes de acordo com a pontuação obtida em baixo, intermediário e alto risco para ECM sendo para a fase hospitalar. Ambos os escores apresentaram acurácia para predizer eventos próxima a $70 \%$. CONCLUSÃO: Baseados em dados do Registro do "mundo real" foi possível desenvolver o Escore DESIRE que permitiu a adequada estratificação do risco de eventos cardíacos maiores após o implante de SF, nas fases hospitalar e tardia. A partir destas estimativas de risco é possível consubstanciar as escolhas terapêuticas.

Descritores: 1. Coronariopatia. 2. Intervenção coronária percutânea. 3. Stents farmacológicos. 4. Fatores de risco. 5. Prognóstico 


\section{SUMMARY}

Moreira, AC. Cardiovascular Risk Stratification for Patients Treated with Drug-eluting Stents: Development and Validation of the DESIRE score \{Thesis\}. São Paulo: Instituto Dante Pazzanese de Cardiology, University of São Paulo; 2017. 166p.

BACKGROUND: Risk stratification before percutaneous coronary intervention is a key element to assist in decision making offering the level of expectations for immediate and late outcomes. This research aims to develop the DESIRE score to predict the risk of major cardiac events (MACE) in patients $(P)$ who underwent drug-eluting stent implantation in daily clinical practice. METHODS: Since 2002 all consecutive patients treated with SF in a single center were included in the non-randomized DESIRE (DrugEluting Stents In the Real world) Registry. We developed the DESIRE score based on a retrospective analysis of the patients included between January 2007 and December 2012, since this population better represented the contemporary practice of interventional cardiology. The associations of the variables with the events were tested by chi-square and Student's $t$ tests. Due to the differences between the analyzed phases, we decided on two models of risk prediction: hospital (Logistic Regression) and late (Cox) estimating the time until the first event. After the development, the score was applied prospectively in the patients included between January 2013 and December 2014. The risk estimates obtained were compared to the observed MACE rates, validating the DESIRE score in the two phases. RESULTS: A total of 4,061 patients were included in the study population. Of these, 2,863 P were the B1 cohort that was used for the development of the score and 1,198 $\mathrm{P}$ in the $\mathrm{B} 2$ cohort in which the score was applied and validated. The variables and their respective points for the hospital DESIRE score ranged from 0 to 37 points were: age in years $\leq 49=0 ; 50$ to $59=1 ; 60$ to $69=2 ; 70$ to $79=4 ; \geq 80=6$ ); Previous myocardial revascularization surgery (1); Peripheral vascular disease (5); Chronic kidney dysfunction (3); Acute coronary syndrome (3); Multivessel disease (3) Saphenous venous graft (6); lesion with thrombus (5) and Long lesion (5);The following variables composed the late DESIRE score (0 to 45 points): Diabetes mellitus with oral medication (4) or with insulin (9); previous myocardial revascularization surgery (2); Peripheral vascular disease (6); Acute coronary syndrome (5); Multivessel disease (4); ejection fraction $<40 \%(6)$; saphenous vein graft (8) and small vessel (5). We defined 3 risk ranges for each of the scores by 
categorizing the patients according to the low, intermediate and high-risk scores for ECM being for the hospital phase. Both scores had accuracy to predict MACE close to $70 \%$.

CONCLUSION: Based on data from the "real world" registry, it was possible to develop the DESIRE score that allowed the adequate stratification of the risk of major cardiac events after the SF implantation in the hospital and late phases. From these risk estimates it is possible to substantiate the therapeutic choices.

Descriptors: 1. Coronary Artery Disease. 2. Percutaneous Coronary Intervention; 3. Drug-eluting Stents 4. Risk factors. 5. Prognosis. 
1 INTRODUÇÃO 
A ocorrência das doenças cardiovasculares (DCV) na população é alta e eleva-se com a idade, haja vista as taxas crescentes de acordo com as faixas etárias no Brasil, em 2008. ${ }^{(1)}$ (Figura 1)

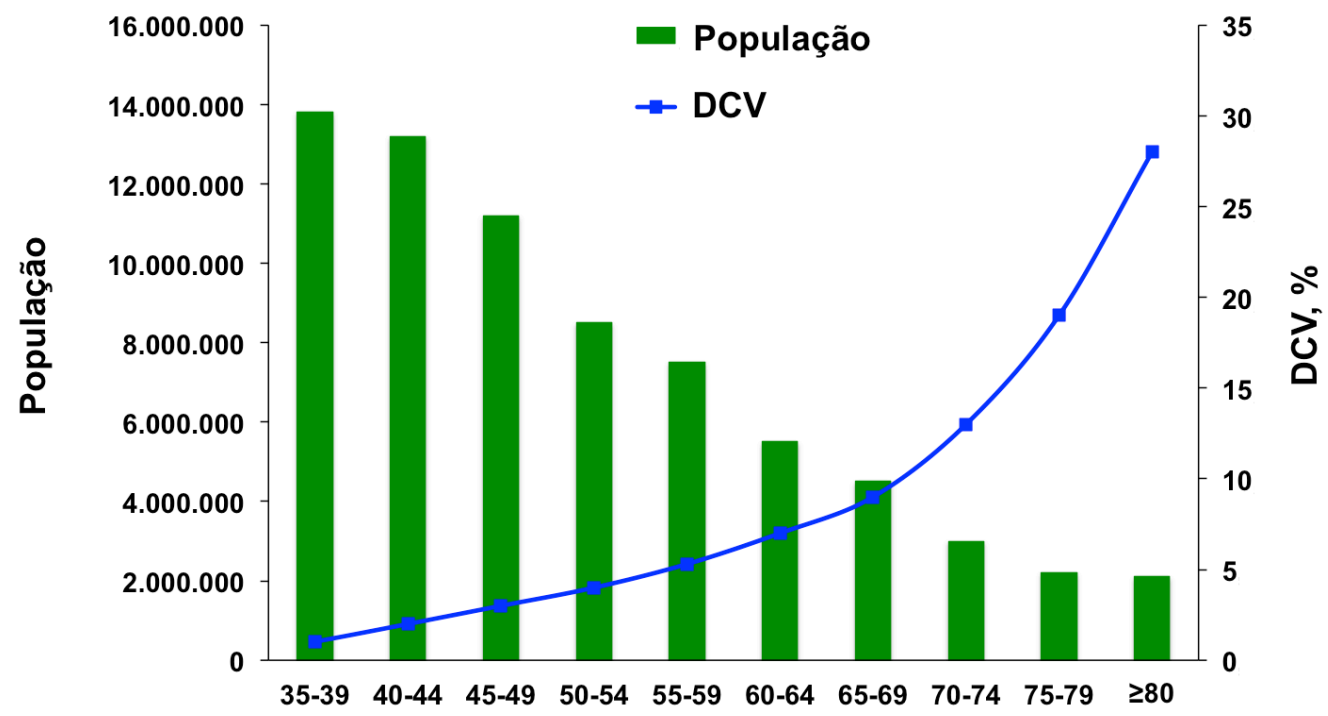

Faixa etária, anos

Figura 1- Taxas de doenças cardiovasculares (linha) na população brasileira de acordo com os grupos etários. DCV = doença cardiovascular. Adaptado de Azambuja e cols., $2008^{(1)}$

Além disso, as DCV constituem a principal causa de óbito na população mundial, tendo sido relacionadas a mais de 17 milhões de mortes em 2013, o que representa $31 \%$ de todos os óbitos registrados naquele ano. ${ }^{(2)}$ No Brasil, pelo menos $20 \%$ das mortes, em indivíduos com mais de 30 anos, são atribuídas a este grupo de doenças. ${ }^{(3,4)}$ 
Entre estas afecções, destaca-se a doença arterial coronária (DAC), responsável por mais da metade dos eventos cardiovasculares em homens e mulheres acima dos 75 anos. Para exemplificar, estima-se que, nos EUA, ocorra um infarto do miocárdio a cada 42 segundos. ${ }^{(5)}$

Sobretudo no mundo ocidental, as inúmeras medidas implementadas com o intuito de conter este grande impacto negativo da DAC conseguiram reduzir as taxas de eventos relacionados à DAC, ${ }^{(5)}$ porém, ainda de maneira insatisfatória e com grande variação regional. No nosso País, a tendência à redução da taxa de mortalidade por DAC cessou a partir de 2007, como se verifica na figura $2 .{ }^{(6)}$
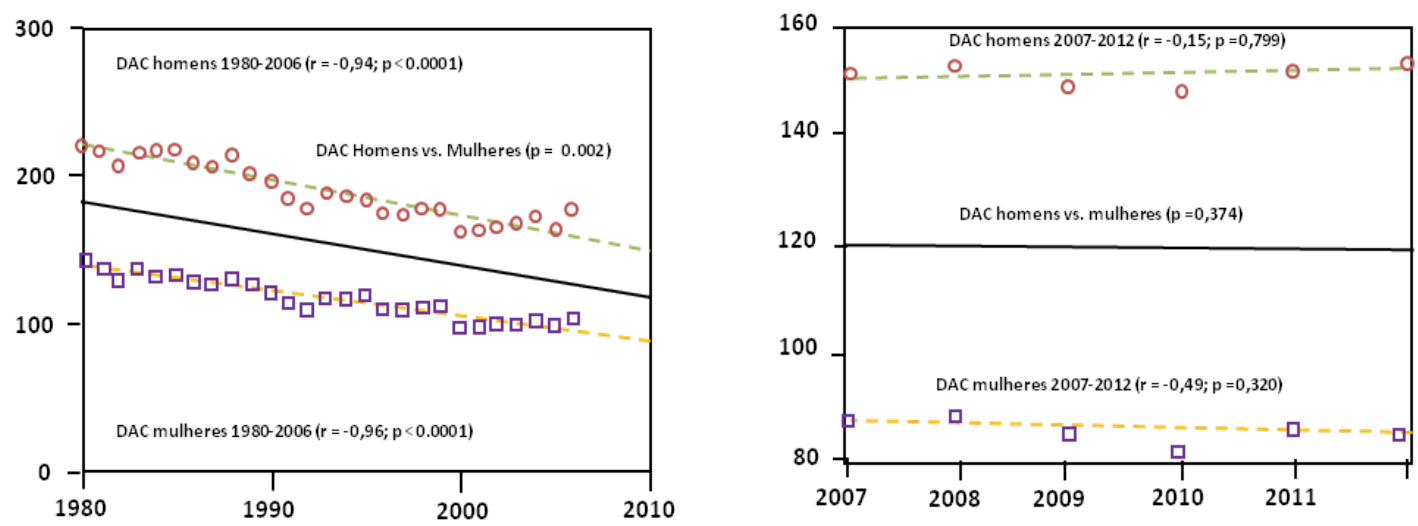

Figura 2 - Análise de regressão linear simples e comparação das linhas de regressão, entre homens e mulheres, da mortalidade por doença arterial coronária (DAC) para os períodos de 1980 a 2006 e de 2007 a 2012. Adaptado de Mansur e cols., $2016{ }^{(6)}$

A DAC, além de estar relacionada a um grande número de mortes, também exerce um impacto econômico-financeiro mundial muito negativo. ${ }^{(7)}$ O aumento expressivo nos custos dos tratamentos médicos, inclusive 
naqueles devidos às internações hospitalares, é motivo de grande preocupação, sobretudo para a Saúde Pública. Se considerarmos apenas os procedimentos cardiovasculares, entre 2000 e 2010 houve um incremento de $28 \%$ no número dos mesmos nos Estados Unidos, que alcançaram 7.588.000 intervenções percutâneas e cirúrgicas ao final da década. ${ }^{(5)}$

Particularmente, com relação à DAC, os custos diretos e indiretos foram estimados em 207,3 bilhões de dólares naquele País, entre 2011 e 2012 (MEPS, NHLBI), sendo que esta representa cerca de dois terços dos gastos, em relação às demais DCV combinadas. ${ }^{(5)}$ (Figura 3)

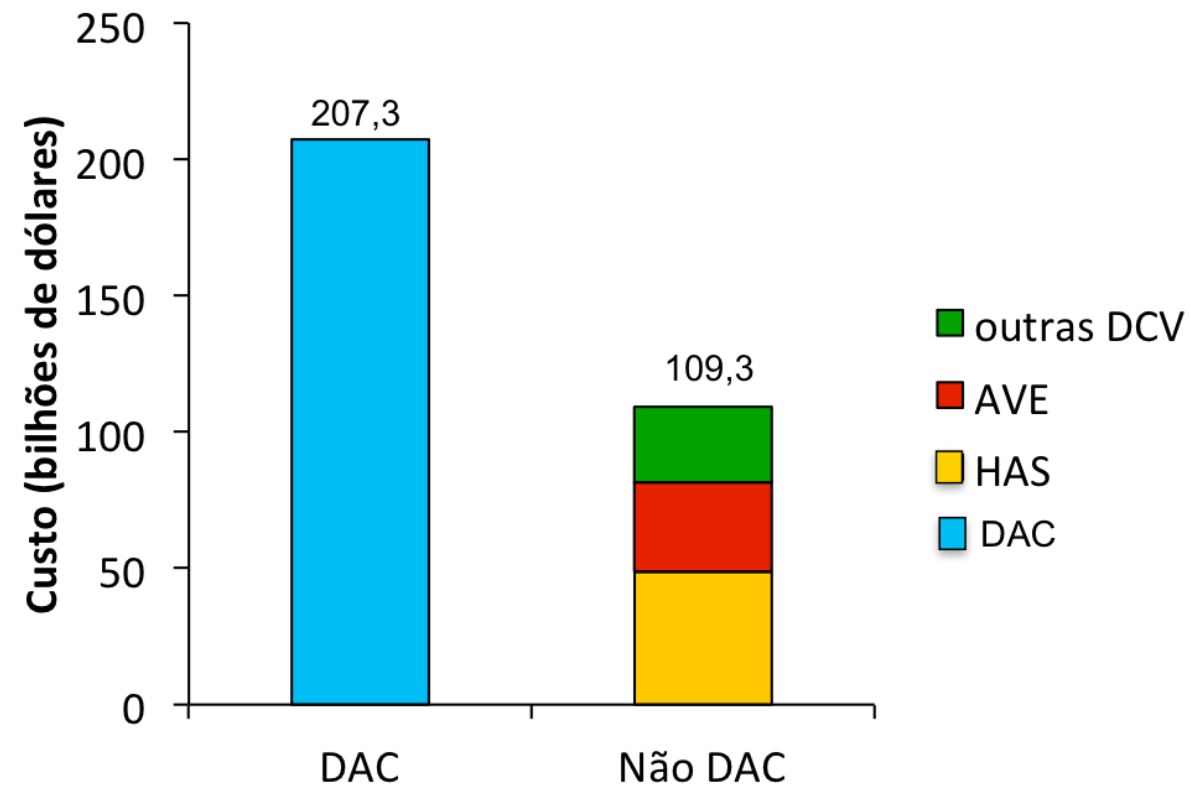

Figura 3- Custos diretos e indiretos para DCV nos EUA entre 2011 e 2012. Fonte: National Heart, Lung and Blood Institute. AVE= acidente vascular encefálico; DAC=doença arterial coronária $\mathrm{DCV}=$ doenças cardiovasculares; HAS=hipertensão arterial sistêmica;. Adaptado de Mozaffarian e cols. ${ }^{(5)}$

Esse mesmo fenômeno é verificado no Brasil, nos anos recentes. ${ }^{(1,8)}$ 
Assim sendo, além do aumento no número dos procedimentos, entre eles os de revascularização miocárdica, sobressaem-se também os altos investimentos necessários às constantes inovações tecnológicas, em especial no campo das intervenções coronárias percutâneas (ICP).

\subsection{Evoluções da intervenção coronária percutânea}

Introduzida em 1977 por Andreas Gruentzig $^{(9)}$, a angioplastia transluminal coronária (ATC) tornou-se a forma de revascularização miocárdica mais aplicada em todo o mundo, sendo na maioria dos bancos de dados, pelo menos duas a três vezes mais prevalente que os procedimentos cirúrgicos. ${ }^{(5)}$

No nosso Serviço no Hospital do Coração - ASS (HCor - ASS), em São Paulo-SP, por exemplo, os procedimentos percutâneos constituíram, em 2016, mais de 90\% das revascularizações miocárdicas, quando comparados ao tratamento cirúrgico $(\mathrm{CRM}){ }^{(10)}$ (Figura 4) 


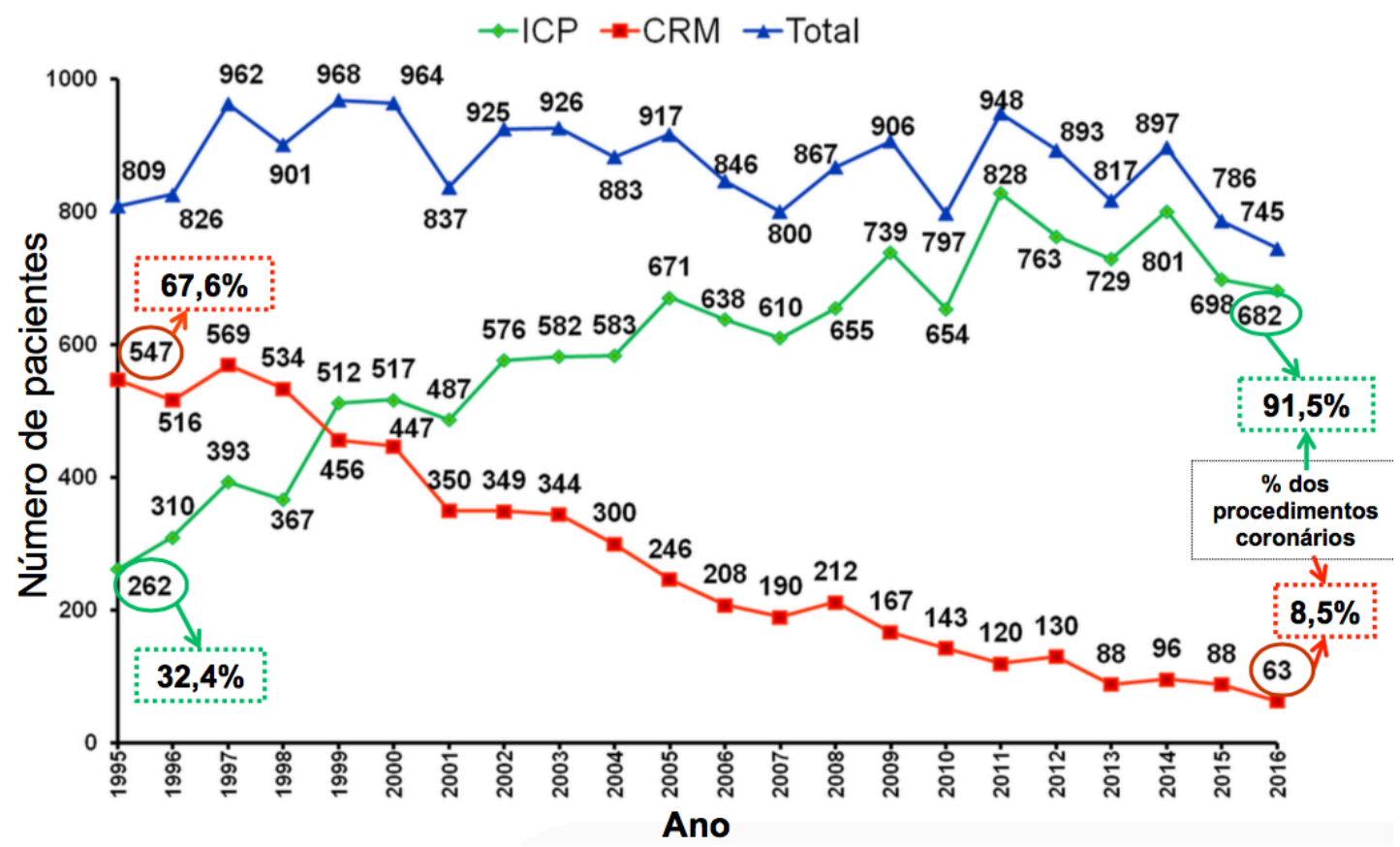

Figura 4 - Evolução comparativa das formas de revascularização miocárdica (ICP e CRM) ao longo dos anos, no Hospital do Coração da Associação do Sanatório Sírio (HCor-ASS). ICP=intervenção coronária percutânea; $\mathrm{CRM}=$ cirurgia de revascularização miocárdica. Adaptado de Costa Jr e cols. ${ }^{(10)}$

Essa progressão se deve, entre outros aspectos, aos melhores resultados das ICP nos últimos anos, em virtude dos constantes avanços tecnológicos, particularmente no campo dos novos instrumentais e em especial à introdução dos stents farmacológicos (SF) em 2002.

O stent coronário balão expansível implantado pela primeira vez, em humanos, por Sousa e cols. $(1987)^{(11)}$, agregou mais segurança e efetividade aos procedimentos percutâneos, até então representados pelo balão e pelas técnicas extracionais.

Constituídas por hastes metálicas, as endopróteses revolucionaram a ICP, por sustentarem a parede arterial, selando as dissecções e eliminando o remodelamento negativo (elástico e fibrótico) pós-ICP, na evolução. Com 
isto, houve: expressiva redução das taxas de oclusão do vaso tratado: praticamente a extinção da cirurgia de emergência e significativa queda da prevalência de reestenose e reintervenções, no médio e longo prazos.

Estes resultados foram aprimorados ainda mais, em 1995, com a contribuição de Colombo e cols. ${ }^{(12)}$. Estes autores introduziram o conceito de "implante ótimo", propondo a pós-dilatação com balões de alta pressão, descartando com isso a necessidade do uso de anticoagulantes, que foram substituídos pela dupla terapêutica antiplaquetária (DTA). ${ }^{(13)}$

Ainda assim, e a despeito da melhoria significativa dos resultados e do aumento da aplicabilidade das $\mathrm{ICP}^{(14)}$, as taxas de reestenose intrastent permaneciam no patamar de 15 a $25 \%{ }^{(15)}$, o que continuou a impulsionar a busca por soluções mais efetivas.

Foram então testadas medicações potentes por via sistêmica; radiações não ionizantes (luz, calor, frio e ultrassom) e ionizantes (radiações $\beta$ e y), com resultados nulos ou modestos, até que surgiu o conceito avançado da "liberação local" de fármacos antiproliferativos.

Em consequência, foi proposta nova biotecnologia: os stents farmacológicos (SF), que passaram a ser avaliados no início deste século, tornando-se os instrumentais mais utilizados nos dias atuais. ${ }^{(5,16)}$

A primeira geração de SF era composta praticamente por dois modelos: um com liberação de sirolimus, o Cypher $^{\circledR}\left(\right.$ Cordis $^{\circledR}$, Warren, NJ, EUA), e o outro, o $\operatorname{TAXUS}^{\circledR}$ (Boston Scientific ${ }^{\circledR}$, Natick, MA, EUA), liberador de paclitaxel. Ao longo do tempo, foram implementados 
aprimoramentos nestes instrumentais, ocorrendo assim a progressiva introdução dos SF de segunda e terceira gerações.

A segunda geração de SF incorporou vários avanços, em relação à primeira, em cada um dos três componentes destes dispositivos, a saber: a plataforma, o polímero e o medicamento a ser liberado. A plataforma de aço inoxidável dos primeiros SF foi sendo substituída, em alguns modelos, pelas ligas de cromo-cobalto ou de platina-cromo, o que permitiu mudanças no desenho e redução na espessura das hastes, conferindo maior navegabilidade aos instrumentais, ainda mantendo boa força radial.

O polímero ou os componentes poliméricos, considerados por muitos os responsáveis pela ocorrência de inflamação crônica e eventualmente trombose protética tardia, experimentaram igualmente grandes evoluções. Os permanentes tornaram-se mais biocompatíveis e foram introduzidos os polímeros absorvíveis, como é o caso do ácido polilático (PLA) e do poli (ácido lático-co-ácido glicólico) $(P L G A)^{(17)}$, que são completamente convertidos em $\mathrm{CO}_{2}$ e $\mathrm{H}_{2} \mathrm{O}$, após três a seis meses do implante.

Mais recentemente, a adição de uma camada microporosa de hidroxiapatita, com espessura de apenas 0,6 $\mu \mathrm{m}$, a uma plataforma metálica de cromo-cobalto, permitiu carrear o fármaco (sirolimus) mesmo na ausência do polímero. Esses modelos já são considerados, por alguns autores, transicionais de segunda para terceira geração. ${ }^{(18)}$

$\mathrm{Na}$ área dos medicamentos antiproliferativos, passaram a ser utilizados, outros fármacos da família "limus", como: o zotarolimus, o everolimus e o biolimus $A 9$, que se mostraram pelo menos tão eficazes 
como o sirolimus no combate à excessiva proliferação de tecido reparativo, senão mais eficientes. ${ }^{(19-25)}$

\subsection{Stents farmacológicos: estudos fundamentais e registros clínicos}

O estudo FIM, conduzido por Sousa e cols., no início dos anos 2000, avaliou de forma pioneira, em humanos, a segurança do stent Cypher ${ }^{\circledR}$, em 30 pacientes estáveis, portadores de doença uniarterial. ${ }^{(26)}$ Além de seguros, mostraram-se altamente eficazes, o que ficou evidenciado pela análise da hiperplasia intimal, nos estudos angiográficos e com ultrassom intracoronário, aos 4, 12, 24 e 48 meses após o procedimento, quando foi detectado tecido reparativo suficiente para a cobertura das hastes, mas de pouca espessura, com ausência de reestenose intrastent, motivando o grande interesse verificado em sequência. ${ }^{(27)}$

Vários estudos clínicos chamados fundamentais foram então conduzidos, para comparar, aleatoriamente, os SF com os metálicos não liberadores de medicamentos.

Esses estudos da série SIRIUS ${ }^{(28-32)}$ e da série $\operatorname{TAXUS}^{(33-39)}$, em populações selecionadas de baixa a moderada complexidade clínica e angiográfica, impulsionaram fortemente o emprego destes dispositivos em todo o mundo. ${ }^{(40-45)}$ 
Posteriormente, já com os SF de segunda e terceira gerações, os estudos clínicos randomizados (ECR), em populações mais complexas, vieram corroborar os achados iniciais e expandiram ainda mais as aplicações dos SF para revascularização miocárdica. ${ }^{(46,47)}$

De grande relevância também foram os Registros clínicos, que refletindo a rotina prática diária, têm contribuído enormemente para a compreensão dos resultados dos SF no "mundo real", no qual frequentemente são utilizados em populações excluídas dos ECR.

Exemplo disso, é o Registro unicêntrico RESEARCH (Rapamycin Eluting Stent Evaluated at Rotterdam Hospital) ${ }^{(48)}$, um dos primeiros com SF, que já demonstrava os excepcionais resultados do stent Cypher $^{\circledR}$, em cenários complexos, como por exemplo em pacientes multiarteriais com lesões longas. As percepções de eficácia e segurança desses dispositivos precederam inclusive os resultados dos ECR, e repetiram-se em outras situações clínicas e angiográficas, como é o caso: dos diabéticos, dos portadores de insuficiência renal, das lesões em bifurcações e em vasos de pequeno calibre.

Outro exemplo eloquente da importância dessas séries é o do Registro e-Cypher ${ }^{(49)}$, no qual uma população de complexidade clínica e angiográfica pelo menos moderada, e não considerada nas diretrizes da época, foi tratada em vários centros de diversos países e continentes, replicando os excelentes resultados iniciais, também precedendo os ECR específicos.

A partir daí, alguns outros registros contribuíram para consolidar o amplo uso destes instrumentais na prática , especialmente nas indicações 
chamadas off-label. Entre eles poderíamos citar os Registros: NHLBI Dynamic, Wake Forest University e SCAARS. ${ }^{(50-53)}$

No Brasil, destaca-se o Registro DESIRE ${ }^{(54)}$ (Drug-Eluting Stents in the Real World), desenvolvido pelo nosso grupo, no HCor-ASS em São Paulo-SP. Trata-se de um estudo prospectivo, de inclusão consecutiva de pacientes, iniciado em 2002, quando os SF foram liberados para o uso clínico, no país. Até dezembro de 2016, 7.074 pacientes foram recrutados, nos quais foram implantados 12.497 SF. A figura 5 mostra a inclusão anual destes casos, cujos resultados têm sido publicados ao longo da última década. ${ }^{(10,55-68)}$

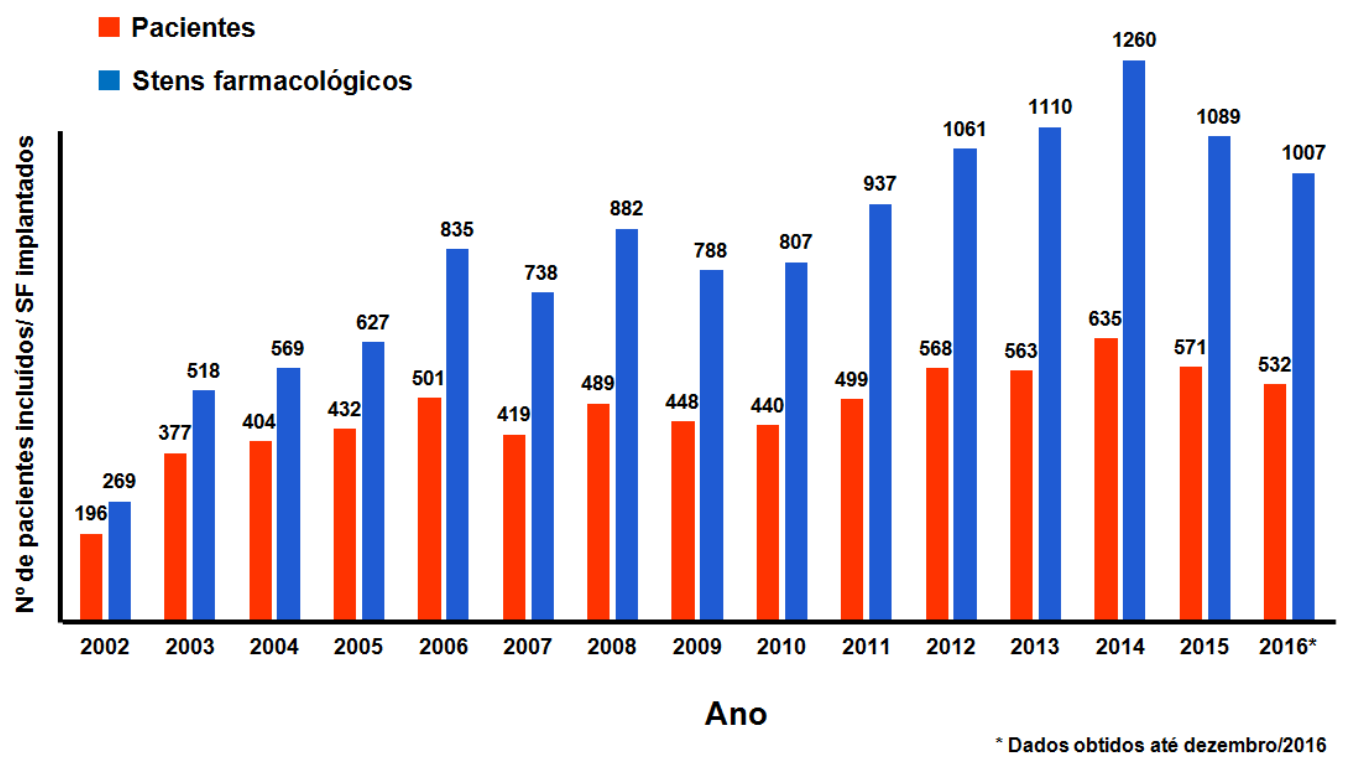

Figura 5- Inclusão consecutiva dos 7.074 pacientes tratados com 12.497 stents farmacológicos (SF) a partir de maio/2002 até dezembro/2016, no Registro DESIRE.

No decorrer destes quinze anos de existência, o Registro DESIRE tem incorporado praticamente todas as evoluções tecnológicas da área, bem 
como os avanços nas indicações, e no protocolo antiplaquetário adjunto, entre outros aspectos que foram sendo implementados na prática, em função das percepções da pesquisa e da própria experiência acumulada.

\subsection{Importância atual dos escores de risco}

A despeito do melhor perfil de segurança e eficiência das ICP, e da consequente redução das taxas de ECM a elas relacionados ${ }^{(16,69)}$, ao longo do tempo, a busca pela excelência dos resultados do tratamento da DAC, com estratégias individualizadas segundo o risco, continua impulsionando a necessidade de prevê-los.

A estratificação do risco individual, facilitada pela aplicação de um escore, é útil em vários aspectos, destacando-se pelo menos três:

$1^{\circ}$. o clínico - na tomada de decisão, e no esclarecimento do paciente, objetivando um consentimento plenamente informado para os procedimentos e otimização do tratamento;

$2^{\circ}$. o investigacional - no delineamento perfeito da população, permitindo comparação de casuísticas distintas em estudos clínicos e

$3^{\circ}$. o regulatório - na avaliação de qualidade do Serviço hospitalar e dos desempenho dos intervencionistas. 
De uma forma mais abrangente, outros aspectos também poderiam ser mencionados, sobre a utilidade de uma ferramenta de estratificação de risco, em situações específicas, como nos casos com SCA. Neste cenário, a identifição dos pacientes com menor risco justificaria a redução no tempo de internação e, possivelmente, implicaria em menor custo dos tratamentos e mais rápida reinserção social. ${ }^{(70)}$ Já nos casos com risco elevado, mudanças estratégicas poderiam redundar em resultados mais favoráveis, minimizando o impacto negativo dos aspectos de complexidade clínica e angiográfica.

\subsection{Os escores de risco para revascularização miocárdica}

A tentativa de identificar pacientes mais propensos à ocorrência de eventos, relacionados ou não às intervenções, é uma busca de longa data ${ }^{(71,72)}$, já precedendo a era dos cateteres. Várias publicações do passado mostram a identificação de preditores prognósticos, em pacientes mantidos em tratamento clínico ou submetidos à revascularização cirúrgica, em diferentes cenários.

Em $1977^{(73)}$, por exemplo, definiram-se alguns dos mais importantes preditores de eventos adversos relacionados à DAC como: o número de artérias ou territórios coronários comprometidos e a quantidade de miocárdio em risco. Demonstrou-se que a habilidade prognóstica dessas variáveis 
aumentava à medida que outras variáveis foram sendo integradas, como por exemplo, o grau de obstrução coronária. Nesta ocasião, surgia o escore Duke Jeopard, validado em 1985. ${ }^{(74)}$

A percepção de que as associações de variáveis poderiam aumentar a capacidade de predição de eventos maiores, em decorrência de efeito aditivo, foi aos poucos se fortalecendo. Um exemplo clássico é o do Estudo $\mathrm{CASS}^{(75-77)}$ que, a despeito de ter sido desenhado para avaliar o efeito da CRM nos desfechos clínicos, trouxe grande contribuição à época, ao demonstrar que o grau de disfunção ventricular esquerda e a extensão da DAC tinham efeitos somados e sinérgicos e, desta forma, sua associação influenciava diretamente na sobrevida dos pacientes. (Figura 5)
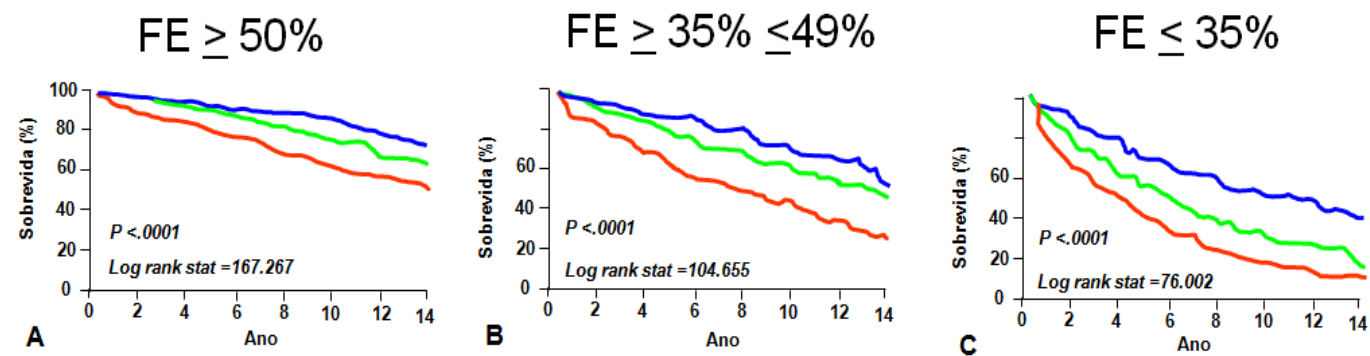

Figura 6 - Sobrevida dos pacientes mantidos em tratamento medicamentoso no Estudo CASS. Em A, pacientes com FE <50\%; B, pacientes com FE entre 35 e $49 \%$, e $\mathrm{C}$, pacientes com $\mathrm{FE} \leq 35 \%$. Número de vasos acometidos $\backsim: 1$, $\sqsubset: 2$ e $\backsim: 3$. $\mathrm{FE}=$ Fração de ejeção. Adaptado de Emond, $1994 .{ }^{(76)}$

Já no campo das ICP, e a partir de 1988, passou-se a utilizar a classificação angiográfica das lesões proposta pela Diretriz do American College of Cardiology e da American Heart Association (ACC/AHA), para prever o risco de insucesso do procedimento com o balão. ${ }^{(78)}$ Esta 
classificação relaciona o tipo de lesão às respectivas taxas de sucesso do procedimento: tipo A acima de $85 \%$, tipo B entre $60 \%$ e $85 \%$ e tipo C menores que $60 \% .^{(78,79)}$ (Tabela 1$)$

Tabela 1 - Classificação das lesões-alvo baseada nas características angiográficas de acordo com o ACC/AHA*

\section{Características específicas da lesão}

- Focal $(<10 \mathrm{~mm})$

- Concêntrica

- Facilmente acessível

- Angulação $<45^{\circ}$

- Contorno liso
Tipo A (taxa de sucesso $>\mathbf{8 5 \%}$; baixo risco)

- Pouca ou nenhuma calcificação

- Não oclusiva

- Não ostial

- Sem envolvimento de ramo importante

- Ausência de trombo
- Tubular (comprimento de 10 a 20mm)

- Excêntrica

- Tortuosidade moderada do segmento proximal

- Segmento proximal com angulação: $>45^{\circ},<90^{\circ}$

- Contorno irregular
60 e $85 \%$; risco moderado**)

- Calcificação moderada a importante

- Oclusão crônica <3 meses

- Lesão aorto-ostial

- Lesões de bifurcação que requerem dois fios - guia

- Algum trombo presente
- Difuso (> 20mm)

Tipo C (taxa de sucesso $<60 \%$; alto risco)

- Tortuosidade excessiva do segmento proximal

- Segmentos proximais extremamente angulados $>90^{\circ}$
- Oclusão crônica $>3$ meses

- Incapacidade de proteger os principais ramos laterais

- Enxertos venosos degenerados

$\left({ }^{*}\right) \mathrm{ACC} / \mathrm{AHA}=$ American College of Cardiology/ American Heart Association; $\left(^{* *}\right)$ Embora o risco de oclusão aguda do vaso seja moderado, em alguns casos a probabilidade de complicações maiores pode ser baixa como: nas oclusões $<3$ meses de ocorrência ou na presença de rica circulação colateral ${ }^{(78,79)}$

Embora idealizada para predizer o sucesso da ICP com o balão, esta forma de estratificar os casos tratados foi revisada e seu uso, na estimativa do resultado imediato do procedimento, avançou para a era dos stents, mesmo quando com estes instrumentais os resultados já eram mais 
previsíveis, em função das próprias características das endopróteses. $(78,80,81)$

Entretanto, por não considerar variáveis clínicas, reconhecidamente relacionadas à ocorrência de desfechos desfavoráveis após as ICP, esta classificação tornou-se limitada, especialmente com a ampliação das indicações das ICP após o início do emprego dos SF.

Diante da ausência de ferramenta estratificadora ampla e contemporânea no campo da ICP, passou-se então à utilização de alguns escores desenvolvidos em populações revascularizadas cirurgicamente, também para casos a serem submetidos às ICP, especialmente quando persistia dúvida em relação à escolha da forma de revascularização a ser indicada, se cirúrgica ou percutânea. ${ }^{(82,83)}$ É o caso do emprego rotineiro do EuroSCORE (desenvolvido para CRM), aos candidatos à ICP.

O escore SYNTAX ${ }^{(84)}$, elaborado mais recente apenas com variáveis angiográficas e calculado a partir de um aplicativo, foi desenvolvido para avaliar população de pacientes com doença coronária complexa e constituise em outro exemplo. Esta ferramenta de estratificação passou a ser mais utilizada na Cardiologia Intervencionista do que na Cirurgia Cardiovascular, por proporcionar uma avaliação padronizada do acometimento arterial pela aterosclerose, expressando a extensão e gravidade da DAC. Entretanto, por não considerar as características clínicas, no algoritmo de avaliação, e não se aplicar a pacientes com CRM prévia, apresenta limitações não desprezíveis. 
Com o intuito de aumentar o poder de discriminar a evolução clínica pós-ICP, considerando também as comorbidades, passou-se então a testar a associação do escore SYNTAX (angiográfico) a vários outros escores, desenvolvidos exclusivamente com variáveis clínicas. ${ }^{(85-87)}$ Como exemplos, podemos citar as experiências publicadas com a associação do escore SYNTAX ao EuroSCORE ${ }^{(85)}$ ou ao escore ACEF. ${ }^{\left({ }^{86}\right)}$

Mais recentemente, o escore SYNTAX II foi desenvolvido, tentando sobrepujar essa carência, reunindo variáveis clínicas e angiográficas, para melhor guiar a escolha entre CRM e ICP, para casos complexos, com acometimento avançado, entretanto, tendo em perspectiva apenas um evento adverso na evolução: a mortalidade até 4 anos de seguimento.

Outros escores também buscaram atender essa necessidade, entretanto fizeram-no de maneira ainda insuficiente, quer por não incluírem todas as variáveis clínicas relevantes, quer por não se aplicarem ao seguimento clínico tardio em todos os seus desdobramentos ou não abordarem todos os desfechos de importância, ou ainda por incluírem variáveis que não são facilmente coletadas. ${ }^{(83,86,88-99)}$

Tendo em vista estas lacunas, e considerando a experiência do nosso grupo, acumulada com o Registro DESIRE há uma década e meia, decidimos por buscar ultrapassar essas limitações, para obter-se então uma estimativa mais precisa e aplicável da predição da ocorrência de eventos cardiovasculares maiores (ECM), na evolução pós-SF. 


\subsection{Hipótese}

O emprego de uma ferramenta de estratificação de risco, desenvolvida e validada em uma população brasileira e contemporânea, tratada com SF, poderia auxiliar na tomada de decisão terapêutica? 
2 OBJETIVOS 


\subsection{Objetivos primários}

Os objetivos primários desta pesquisa são:

a) desenvolver o escore DESIRE, para estimar o risco individual de ocorrência de eventos cardíacos maiores (ECM) combinados, na fase hospitalar e tardia, em pacientes submetidos ao implante de SF contemporâneo.

b) aplicar este escore prospectivamente para validá-lo, nas duas fases, testando sua acurácia.

\subsection{Objetivos secundários}

Determinar, nas populações de desenvolvimento e validação desse escore, nas fases hospitalar e tardia, as taxas de:

a) ECM individualizados:

- óbito cardíaco

- infarto não fatal

- revascularização da lesão-alvo

b) trombose protética. 
3 MÉTODOS 
Esta investigação foi aprovada pelo Comitê de Ética em Pesquisa (protocolo número 4286/2012), sendo o CAAE da Plataforma Brasil:10812512.0.0000.5462.

Após receberem as informações relevantes a respeito do procedimento ao qual seriam submetidos e, mediante a assinatura do termo de consentimento livre e esclarecido, conforme a Declaração de Helsinque e a Resolução 196/96 do Conselho Nacional de Saúde, os pacientes foram habilitados para a inclusão neste estudo.

Por se tratar de um Registro destinado a refletir a prática diária do Serviço de Cardiologia Intervencionista do HCor - ASS São Paulo - SP, os procedimentos, bem como os materiais utilizados, foram subvencionados conforme a rotina vigente na Instituição.

Médicos e enfermeiros pesquisadores, tecnólogos e assistentes de pesquisa, ligados ao Serviço de Cardiologia Intervencionista do HCor-ASS e ao Instituto de Pesquisa desse Hospital, participaram da presente investigação clínica. ${ }^{(100)}$

\subsection{Delineamento do Registro DESIRE}

Os pacientes consecutivamente incluídos no Registro DESIRE, entre maio de 2002 e dezembro de 2016, foram então divididos em três coortes. (Figura 7) 


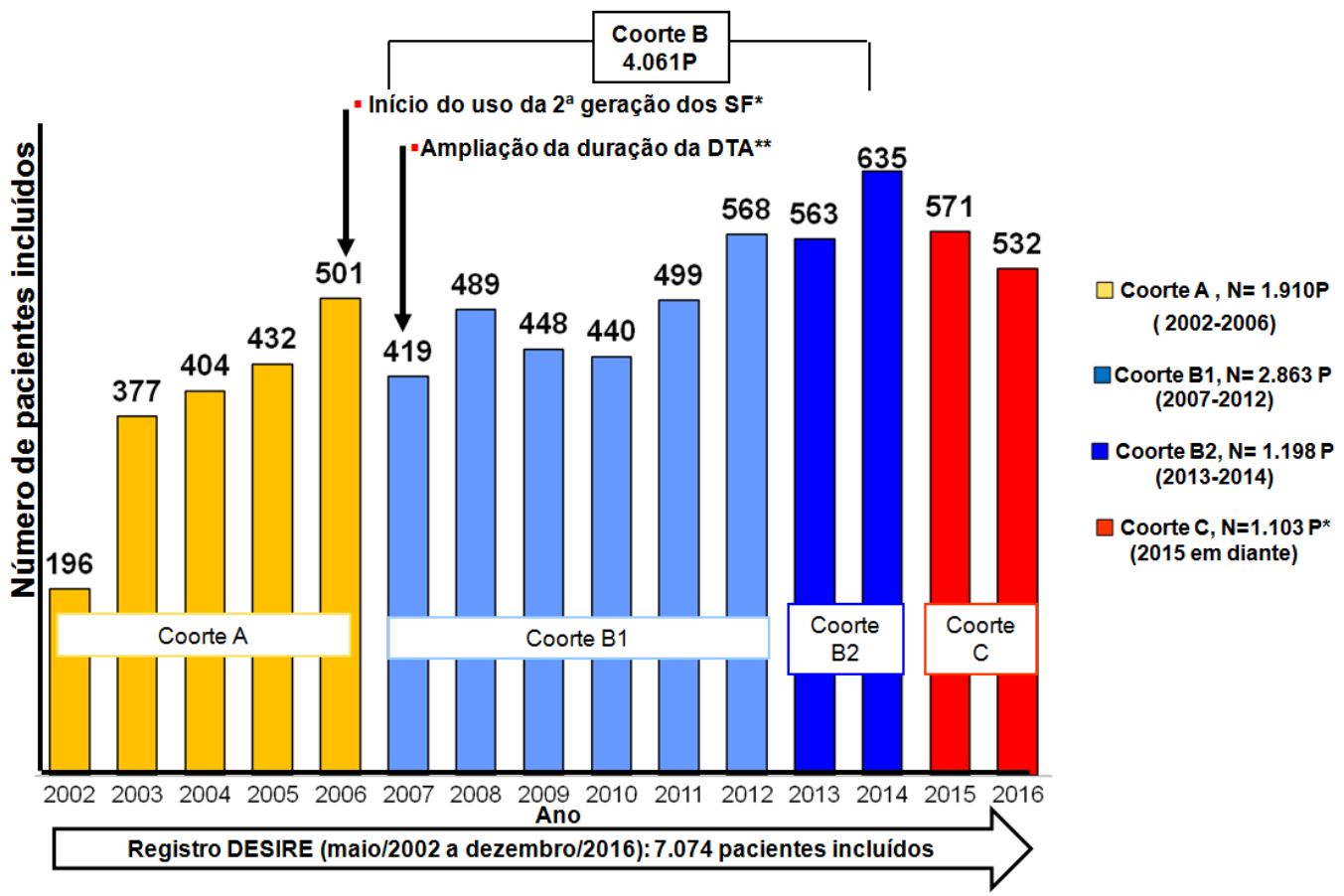

*Dados obtidos até dezembro/2016

Figura 7 - Inclusão consecutiva dos pacientes tratados com SF a partir de 2002, no Registro DESIRE. Em amarelo, a coorte A, que compõe a fase inicial do Registro DESIRE na qual predomina o uso dos SF de $1^{\text {a }}$ geração. Destaca-se, em azul, a coorte B que representa a casuística deste estudo. Em azul claro, os pacientes incluídos de 2007 a 2012, coorte B1, cujos dados foram utilizados para o desenvolvimento do escore DESIRE. Em azul escuro, os pacientes incluídos em 2013 e 2014, coorte B2, amostra destinada à validação do escore. Em vermelho, a coorte $C$, na qual estão inseridos os casos que continuam a ser incluídos, após o término do recrutamento para esta pesquisa. *SF: stents farmacológicos; ${ }^{* *}$ DTA: dupla terapêutica antiplaquetária.

A coorte A (2002-2006) é considerada, hoje, uma amostra histórica sendo composta de casos em que foram empregados os SF de primeira geração. A coorte B (2007-2014), que reflete a prática atual já com o emprego dos SF de segunda e terceira gerações, constitui a população deste estudo, na qual se pode obter pelo menos dois anos de evolução até dezembro de 2016. A inclusão de pacientes no Registro DESIRE continuou, mesmo após o término do recrutamento de pacientes para esta investigação. Os pacientes subsequentes constituem a coorte C. 
A coorte B foi subdividida em dois grupos, quais sejam:

a) coorte B1 (2007-2012), destinada ao desenvolvimento do escore DESIRE (fase hospitalar e tardia) e

b) coorte B2 (2013-2014), na qual o escore DESIRE foi aplicado prospectivamente, visando à sua validação mediante seguimento clínico de até dois anos.

Nesse estudo foram analisados os dados clínicos, angiográficos e técnicos do procedimento, bem como os dados evolutivos, por meio do preenchimento de fichas específicas. A digitação destes dados foi sempre feita por um profissional habilitado para esta função, assim como a captação dos dados evolutivos.

\subsection{Casuística}

\subsubsection{Critérios de inclusão}

Para o desenvolvimento do escore DESIRE foram incluídos pacientes da rotina do Registro DESIRE, entre janeiro de 2007 e dezembro de 2012 (coorte B1 - figura 7), com as seguintes características:

a) com indicação para revascularização miocárdica

b) apropriados anatomicamente para implante de stent

c) submetidos à ICP em nosso Serviço com o uso exclusivo de SF. 
Para a validação do escore DESIRE, foram incluídos os pacientes subsequentes à coorte $\mathrm{B} 1$, recrutados a partir de janeiro de 2013 até dezembro de 2014 (coorte B2), com os mesmos critérios de inclusão e, submetidos às mesmas estratégias de manejo clínico, técnico e de acompanhamento evolutivo. (Figura 7)

\subsubsection{Critérios de exclusão}

Foram excluídos os pacientes que apresentassem:

- idade fértil, teste de gravidez positivo

- hipersensibilidade aos medicamentos que compõem a DTA

- distúrbios hematológicos

- acidente vascular encefálico (AVE) hemorrágico nos últimos seis meses

- sangramento grave recente, em especial gastro-intestinal e/ou gênito-urinário nos últimos seis meses

- previsão de serem submetidos a algum procedimento cirúrgico com brevidade, que impossibilite o uso da DTA por pelo menos um ano

- comorbidade relacionada à expectativa de sobrevida menor que um ano, como por exemplo, alguns tipos de neoplasias e doença pulmonar ou renal avançadas 
- incapacidade sócio-econômica e/ou cognitiva para manter o uso prolongado do protocolo antiplaquetário.

Também foram excluídos os que se recusaram a assinar o termo de consentimento livre e esclarecido.

\subsection{Métodos}

\subsubsection{Preparo do paciente, aspectos técnicos do procedimento e protocolo medicamentoso adjunto}

Os pacientes foram avaliados clinicamente e por meio de exames complementares. O eletrocardiograma foi realizado em três momentos distintos: na admissão do paciente, logo após o procedimento e antes da alta hospitalar. Os marcadores de necrose miocárdica (CK-MB massa e Troponina I ultrassensível) foram avaliados antes do procedimento, seis a doze horas após o término do mesmo e, caso o resultado estivesse acima do percentil 99 do limite superior do valor de referência, outras coletas foram feitas, a cada seis horas, até a verificação da normalização dos valores. Além destes, hemograma, ureia e creatinina foram analisados antes da ICP e, pelo menos, mais uma vez pré-alta hospitalar. 
A DTA, foi iniciada idealmente 24 horas antes do procedimento, exceto nos casos nos quais a ICP foi realizada em caráter de urgência, como em vigência de infarto do miocárdio com supradesnivelamento do segmento ST.

O protocolo antiplaquetário recomendado constou do uso do ácido acetil salicílico, com dose de ataque de $200 \mathrm{mg}$ e dose de manutenção de $100 \mathrm{mg} / \mathrm{dia}$ indefinidamente, associado a um tienopiridínico de segunda ou de terceira geração durante um ano(clopidogrel: dose de ataque $-600 \mathrm{mg}$ seguida de dose de manutenção de $75 \mathrm{mg} /$ dia ou prasugrel: dose de ataque de $60 \mathrm{mg}$ seguida de dose de manutenção de 5 a $10 \mathrm{mg} / \mathrm{dia}$ ); ou então a um antagonista seletivo do receptor $\mathrm{P}_{2} \mathrm{Y}_{12}$ do ADP, como o ticagrelor (dose de ataque de $180 \mathrm{mg}$ e dose de manutenção de $90 \mathrm{mg}$ duas vezes/dia).

Antes de iniciar o procedimento, foi administrada por via intravenosa, a heparina não fracionada (100 Ul/kg). Nos casos não frequentes de uso do inibidor da glicoproteína Ilbllla, a dose da heparina foi reduzida para $70 \mathrm{Ul} / \mathrm{kg}$.

Em casos de alergia a um dos antiplaquetários ou ao meio de contraste radiológico, assim como em presença de diabete melito ou de insuficiência renal, utilizaram-se protocolos específicos de dessensibilização ou de proteção, conforme descrito em publicações anteriores. ${ }^{(101)}$

As escolhas do SF a ser implantado, bem como do uso dos métodos invasivos adjuntos, como por exemplo: o ultrassom intracoronário e a tomografia de coerência óptica, foram deixados a critério do operador.

Os procedimentos foram realizados objetivando o resultado angiográfico ótimo. Sempre que necessário, procedeu-se à pré-dilatação. 
Cuidou-se para que a pós-dilatação, quando indicada, fosse praticada com balões mais curtos que o SF implantado, evitando-se assim dano nos segmentos adjacentes (bordas). Tivemos como escopo sempre a cobertura completa da lesão e, para que esta se verificasse, sempre que necessário, um segundo SF foi implantado, com sobreposição de suas hastes às do primeiro SF, evitando-se espaços não cobertos entre os dois dispositivos.

\subsubsection{Análise pela angiografia coronária}

As análises qualitativa e quantitativa dos dados da angiografia coronária foram realizadas sistematicamente, em pelo menos dois momentos distintos: antes e logo após o procedimento. Sempre que houvesse uma nova coronariografia disponível no seguimento, os dados angiográficos evolutivos também foram registrados e analisados. ${ }^{(102)}$

Alguns critérios foram estabelecidos, a fim de uniformizar e otimizar as análises, como por exemplo: a administração sistemática de nitrato por via intracoronária, antes da aquisição das imagens e a realização de pelo menos duas projeções separadas por mais de $30^{\circ}$, gravadas com 12 a 30 quadros por segundo. Além disso, a análise pós-procedimento foi feita nas mesmas projeções utilizadas na avaliação inicial.

Os filmes angiográficos foram gravados em formato digital $\mathrm{DICOM}^{\circledR}$ (Digital Imaging and Communications in Medicine ${ }^{\circledR}$ ) e arquivados em mídia tipo CD-R. 
A análise inicial qualitativa visava à:

a) avaliação do grau de acometimento pela DAC ou determinação da extensão da doença coronária, definindo o número de territórios miocárdicos acometidos, ou seja, que apresentassem lesão obstrutiva $\geq 50 \%$. Foi também definida a localização das obstruções de acordo com a classificação proposta pelo CASS $^{(103)}$

b) procedeu-se ainda à descrição morfológica do vaso-alvo e da lesão-alvo, identificando características que definissem a complexidade anatômica, entre outras: presença de ectasia ou aneurisma, tortuosidade e/ou angulações excessivas do vasoalvo no segmento proximal à lesão e também presença de calcificação, lesões reestenóticas ou em bifurcação, oclusão crônica e lesões em pontes de safena.

A partir daí, seguiu-se com a caracterização das lesões, de acordo com a classificação do American College of Cardiology (ACC) e Americam Heart Association (AHA), categorizando-as em: lesões tipo A, B1, B2 e C. ${ }^{(78)}$

As bifurcações foram analisadas de acordo com a classificação de Medina. ${ }^{(104)}$

Após o procedimento, foram documentadas situações adversas como, por exemplo: presença de espasmo, dissecção ou trombo na lesão-alvo e em suas imediações, perfuração do vaso-alvo, lentificação do fluxo anterógrado ou mesmo oclusão do vaso tratado. 
Adicionalmente, nas angiografias realizadas no período evolutivo, as lesões reestenóticas foram divididas em quatro tipos, seguindo-se as proposições de Mehan e cols. ${ }^{(105)}$ : focal (< 10mm); difusa (> 10mm, envolvendo apenas o segmento intrastent); proliferativa ( $>10 \mathrm{~mm}$, quando presente no segmento intrastent e nas bordas) e oclusiva.

As mensurações da angiografia quantitativa foram realizadas com o programa de computador validado e disponível comercialmente: QAngio ${ }^{\circledR}$ XA versão 7.2 (Medis Medical Imaging Systems BV ${ }^{\circledR}$, Leiden, Holanda), por profissionais treinados e experientes com o método.

Os dados quantitativos analisados antes do procedimento foram:

a) extensão da lesão (em $\mathrm{mm}$ ): definida como a distância entre os pontos de transição do segmento estenótico às referências normais proximal e distal. De acordo com a extensão da lesão, esta foi classificada como: focal (menor que $10 \mathrm{~mm}$ ), tubular (10 a $20 \mathrm{~mm}$ ), ou difusa (maior que $20 \mathrm{~mm}$ ), para a caracterização da lesão-alvo segundo a classificação ACC/AHA. Para finalidade de desenvolvimento e validação do escore, consideramos lesões longas como $\geq 20 \mathrm{~mm}$

b) referências normais do vaso (em $\mathrm{mm}$ ) foram obtidas nos segmentos proximal e distal à lesão-alvo (10 mm). O diâmetro interpolado do vaso (DIV), em milímetros (mm), foi utilizado para a análise segmentar 
c) o diâmetro mínimo da luz (DML)

d) o porcentual de estenose (\% ED), que foi calculado segundo a fórmula:

$$
\begin{aligned}
& \% E D=[1-(D M L / D I V)] \times 100 \\
& \text { na qual } \\
& \text { \%ED = porcentual de estenose; } \mathrm{DML}=\text { diâmetro mínimo da luz } \\
& \text { e DIV = diâmetro interpolado do vaso. }
\end{aligned}
$$

Após o procedimento, foram realizadas novamente as medidas já descritas, sendo que o DML e o DIV foram determinados também nas porções intra-stent e nas bordas proximal e distal $(5 \mathrm{~mm})$ ao mesmo.

O ganho imediato $(\mathrm{GI})$, após o implante do SF, foi quantificado pela diferença entre o DML pós-procedimento e o DML pré-procedimento, e expresso em $\mathrm{mm}$.

Nos casos submetidos ao reestudo angiográfico, foram analisados, adicionalmente:

a) a perda tardia da luz (PT) em mm, representada pela perda do DML intrastent e nas suas bordas $(5 \mathrm{~mm})$ sendo, calculada pela seguinte fórmula:

\section{PT $(\mathrm{mm})=\mathrm{DML}$ pós-procedimento $-\mathrm{DML}$ tardio} na qual

$$
\mathrm{PT}=\text { perda tardia da luz e DML = diâmetro mínimo da luz. }
$$


b) o ganho líquido (GL) em $\mathrm{mm}$, representando o benefício global, obtido com o implante do SF, em relação ao DML, foi expresso em milímetros e calculado pela seguinte fórmula:

$$
\begin{aligned}
& \qquad \mathrm{GL}(\mathrm{mm})=\mathrm{GI}-\mathrm{PT} \\
& \mathrm{GL}=\text { ganho líquido; } \mathrm{Gl}=\text { ganho imediato e } \mathrm{PT}=\text { perda } \\
& \text { tardia da luz. }
\end{aligned}
$$

$\mathrm{Na}$ fase tardia, a reestenose binária foi definida como a presença de porcentual de ED maior ou igual a $50 \%$ no interior do stent e nas bordas até $5 \mathrm{~mm}$ do término dos hastes do stent, no reestudo angiográfico.

\subsubsection{Seguimento clínico}

Foi sempre realizado pelo médico de referência. O seguimento de 1, 6 e 12 meses após a ICP e, anualmente a partir de então, foi feito por meio de

a) na avaliação presencial eletiva (consultas programadas) ou de urgência e/ou

b) entrevista com um profissional habilitado, por telefone (médico assistente e/ou profissional da Saúde treinado para esta finalidade) e/ou 
c) questionários enviados pelo nosso Serviço de acompanhamento clínico.

Apurou, durante a captação dos dados evolutivos, evidência clínica e/ou por métodos detectores de isquemia, da recorrência da lesão tratada, assim como de progressão da doença coronária, em eventuais reestudos angiográficos.

Os ECM foram pesquisados especificamente, buscando-se sempre a documentação de suas ocorrências.

Sempre que nova coronariografia tenha sido realizada e, diante da disponibilidade da mesma, a angiografia coronária quantitativa foi obtida.

Todos os dados foram registrados numa ficha específica e inseridos no banco de dados.

Todos os eventos adversos identificados foram adjudicados, por pelo menos dois membros do Comitê de Adjudicação de Eventos do nosso Registro, composto pelo investigador principal do Registro DESIRE (JES) e por pelo menos mais um dos pesquisadores do grupo (AM, JRCJ, RC, AS).

\subsubsection{Banco de dados}

Em 2002, uma equipe com experiência prévia em sistemas na área médica elaborou, com a contribuição dos pesquisadores do Registro 
DESIRE, um banco de dados dedicado especificamente à catalogação dos dados deste estudo.

Com o intuito de viabilizar a elaboração de relatórios descritivos, bem como de exportar as informações obtidas em formato adequado para análise estatística, foram criados diversos módulos com as informações referentes aos vários segmentos da análise: dados demográficos e apresentação clínica, aspectos técnicos dos procedimentos; características do vaso-alvo, da lesão-alvo e do SF implantado; eventos adversos e os variados aspectos da evolução tardia.

Ao longo da sua existência, o sistema tem sofrido diversas atualizações, ajustando-se assim às necessidades operacionais e investigativas como: adequação de relatórios, inserção de novas informações e exportações de dados. ${ }^{(106)}$

Desenhou-se um módulo especialmente destinado para atender à demanda das pesquisas e também para dar suporte à administração das informações contidas na base, que permitiram acompanhar a produtividade do cadastramento, servindo também como ferramenta voltada à verificação dos dados.

Os dados foram obtidos e inseridos no sistema por profissionais habilitados, sob supervisão dos médicos pesquisadores, visando a assegurar a integridade das informações.

O sistema desenvolvido na linguagem Visual Basic $\left(\right.$ Microsoft $^{\circledR}$ ) com o banco de dados SQL Server 2008 (Microsoft $^{\circledR}$ ) encontra-se disponível em 
rede local e hospedado em um data center fora do hospital, com acesso por meio da Internet.

Atualizações são encaminhadas e realizadas diretamente por meio da rede local.

\subsubsection{Variáveis analisadas para o desenvolvimento do escore DESIRE e suas definições}

Vinte e sete variáveis foram testadas, das quais quinze foram clínicas e as outras doze, angiográficas. Todas elas foram testadas para as duas fases: hospitalar e tardia. Diante da impossibilidade de se obter informações a respeito de determinada variável, consideramos a mesma inexistente.

\section{A. Variáveis clínicas}

1. Idade: foram incluídos pacientes com 18 anos ou mais, sem limite superior de exclusão. A idade foi sempre referida em anos completos

2. Gênero: inseridos pacientes de ambos os sexos

3. Antecedente familiar para DAC: de acordo com relato do paciente de presença de DAC nos parentes de primeiro grau

4. Diabete melito (DM): diante do relato do paciente de diagnóstico prévio por profissional médico, estando em observação de dieta específica, uso de hipoglicemiante oral e/ou de insulina, ou achados 
hospitalares que identificassem o diagnóstico de acordo com as Diretrizes da Sociedade Brasileira de Diabetes

5. Hipertensão arterial sistêmica (HAS): mediante informação do paciente de diagnóstico médico feito previamente, podendo ou não estar em uso de medicamentos anti-hipertensivos. Na ausência de diagnóstico prévio, caracterizamos o paciente como hipertenso de acordo com os níveis de pressão arterial baseados na Diretrizes Brasileiras de Hipertensão

6. Dislipidemia: de acordo com a descrição do paciente de ter apresentado em exame laboratorial prévio, com preparo adequado, elevação dos níveis de colesterol e/ou triglicérides, acima dos valores normais, não sendo obrigatório o tratamento atual com dieta e/ou com medicamentos hipolipemiantes para a definição desta condição

7. Tabagismo: consideramos: tabagista atual - todo indivíduo que fumasse regularmente, qualquer número de cigarros por dia; não fumante - o que nunca fumou e ex-fumante - aquele que abandonou o hábito de fumar há mais de um ano

8. Obesidade: classificada de acordo com o cálculo do índice de massa corpórea (IMC) e definida como IMC $\geq 30 \mathrm{~kg} / \mathrm{m}^{2}$, utilizando-se dados antropométricos obtidos na internação atual

9. Infarto do miocárdio (IM) prévio: relato do paciente da ocorrência deste evento há mais de 30 dias, tendo sido diagnosticado por médico e/ou pela verificação da presença de novas ondas $Q$ em mais de uma 
derivação, no eletrocardiograma de admissão em determinada área correspondente a uma parede miocárdica

10. Intervenção coronária percutânea prévia: de acordo com a informação do paciente ou mediante apresentação de laudo médico ou de estudo angiográfico comprobatório

11. Cirurgia de revascularização do miocárdio prévia: frente ao relato do paciente e confirmada pelo relatório cirúrgico e/ou pelo estudo angiográfico realizado

12. Acidente vascular encefálico (AVE) prévio: presença de história clínica da ocorrência deste evento, diante ou não de sequela ao exame físico

13. Doença arterial periférica: relato do paciente do diagnóstico médico ou do tratamento de obstruções arteriais extracardíacas e/ou de aneurisma da aorta. Na ausência destes, e frente a uma suspeita no exame físico, exames de imagem foram solicitados, com o intuito de definir a presença desta afecção

14. Insuficiência renal: caracterizada pela elevação dos níveis séricos de creatinina ( $\geq 1,5 \mathrm{mg} / \mathrm{dl}$ ) e/ou do Clearance de creatinina inferior a 60 $\mathrm{mL} / \mathrm{min}$, calculado pela fórmula de Cockcroft \& Gault, sendo para os homens:

Clearance de creatinina $=\frac{(140-\text { idade }) \times \text { peso }(\mathrm{kg})}{\text { creatinina sérica } \times 72}$

e para as mulheres:

Clearance de creatinina $=\frac{(140 \text {-idade }) \times \text { peso }(\mathrm{kg})}{\text { creatinina sérica } \times 72} \times 0,85$ 


\section{Apresentação clínica}

a. Isquemia silenciosa: paciente assintomático apresentando teste detector de isquemia miocárdica positivo

b. Angina estável: relato de dor típica e classificada de acordo com o proposto pela Sociedade Canadense de Cardiologia ${ }^{(107)}$

c. Síndrome coronária aguda: ocorrência de angina instável ou de infarto agudo do miocárdio

- Angina instável: surgimento de dor retroesternal típica.

Definida pela classificação de Braunwald ${ }^{(108)}$

- Infarto do miocárdio recente: registro médico de ocorrência deste evento $\leq 30$ dias. Caracterizado por pelo menos duas das alterações a seguir: dor típica, eletrocardiograma evidenciando alterações do segmento ST ou surgimento de bloqueio do ramo esquerdo, ou de novas ondas $Q$ e elevação dos marcadores de necrose miocárdica (CK-MB massa ou Troponina I), duas vezes acima do valor de referência.

\section{B. Variáveis angiográficas}

Consideramos o paciente portador de DAC:

\section{Extensão da DAC}


a. Uniarterial: quando o estudo angiográfico pré-ICP evidenciou apenas um vaso epicárdico com lesão $\geq 50 \%$, pela avaliação visual

b. Multiarterial: diante de lesão $\geq 50 \%$ em mais de uma artéria coronária irrigando territórios distintos, pela avaliação visual

A seguir, avaliou-se:

2. Função ventricular esquerda (FVE): estimada pela fração de ejeção (FE), que foi medida pela angiografia quantitativa do ventrículo esquerdo, por meio do programa de computador: QAngio ${ }^{\circledR}$ XA versão 7.2 Medis Medical Imaging Systems $\mathrm{BV}^{\circledR}$, Leiden, Holanda. Caso a angiografia do VE não estivesse disponível, um ecocardiograma era solicitado, utilizando-se, para avaliação da FVE, o método de Simpson. Classificamos e avaliamos a variável FVE de duas formas: a primeira, distribuindo as FE em quatro categorias, de acordo com o valor obtido: normal (FE $\geq 55 \%$ ), déficit discreto (FE $\geq$ $40 \%$ e $<55 \%$ ), déficit moderado (FE $\geq 30 \%$ e $<40 \%$ ) e déficit importante (FE < 30\%). A segunda, em dois grupos: normal ou déficit discreto ( $F E \geq 40 \%$ ); déficit moderado ou importante ( $F E<40 \%$ )

3. Vaso-alvo: referindo-se às artérias coronárias nativas acometidas pela DAC e motivo de indicação do SF, incluindo seus ramos: artéria descendente anterior (DA), artéria circunflexa (CX), coronária direita (CD) e tronco da coronária esquerda. Também foram consideradas as pontes de veia safena, quando pelo menos uma lesão-alvo estivesse 
localizada no segmento venoso do enxerto. Adicionalmente, foram consideradas as associações entre os vasos-alvo e, também, a quantidade de vasos tratados (um vaso, dois vasos, três vasos etc.)

\section{Lesão de novo ou reestenose intrastent}

a. Lesão de novo (primária): localizada em um segmento do vaso-alvo que não tivesse sido tratado previamente com qualquer técnica percutânea

b. Reestenose intrastent: lesão $>50 \%$ dentro do stent implantado previamente e/ou nas suas bordas $(5 \mathrm{~mm})$ proximais e distais

5. Lesão ostial: pelo menos uma lesão-alvo localizada na origem da artéria coronária direita ou no tronco da coronária esquerda, da aorta

6. Bifurcação: definida pela presença de estenose $\geq 50 \%$ no vaso principal e/ou no vaso lateral, sendo este de diâmetro $\geq 2,0 \mathrm{~mm}$ (estimativa visual), considerando todos os subtipos da classificação de Medina ${ }^{(104)}$

7. Oclusão crônica: caracterizada pela ausência de fluxo anterógrado no segmento distal à obstrução e evidência desta ocorrência há pelo menos 3 meses

8. Trombo: presença de defeito de enchimento na lesão-alvo ou imediatamente distal a mesma, em mais de uma projeção ou ainda, a observação de embolização de imagem com essas características, para o segmento distal do vaso 
9. Calcificação: caracterizada pela opacificação persistente da artéria coronária na lesão-alvo, evidenciada em mais de uma projeção. Classificada como: discreta, quando fosse única; moderada quando as opacificações persistentes fossem múltiplas e, intensa quando além de múltiplas, essas imagens ocupassem toda a circunferência do vaso na lesão-alvo.

10. Classificação da lesão-alvo: de acordo com os critérios do ACC/AHA. Testadas no modelo de duas formas: a primeira, classificando as lesões-alvo em A, B1, B2 e C, e a segunda, agrupando-as em A ou B1 e B2 ou C

11. Vaso de pequeno calibre: avaliado vaso-alvo com diâmetro $\leq 2,5 \mathrm{~mm}$, pela $\mathrm{ACQ}$

12. Lesão longa: lesão-alvo com extensão $\geq 20 \mathrm{~mm}$, analisada pela $A C Q$.

\subsubsection{Definições dos desfechos}

\section{Sucesso do procedimento}

Definido como a obtenção de resultado angiográfico ótimo, ou seja: lesão residual < 10\% (definida pela ACQ), fluxo coronário TIMI 3 e ausência de trombos intraluminais e lou dissecções residuais.

\section{Sucesso clínico}


Ausência de ECM na fase hospitalar, tendo-se obtido sucesso do procedimento.

\section{Eventos cardíacos maiores}

Os ECM combinados foram definidos como o composto de: óbito cardíaco, infarto do miocárdio não fatal e revascularização da lesão-alvo.

Para caracterização do evento clínico, definimos uma hierarquização dos ECM quanto à gravidade: revascularização da lesão-alvo < infarto não fatal < óbito cardíaco. Assim, diante da ocorrência de mais de um ECM, consideramos sempre o mais grave para a definição do desfecho composto.

Para a análise dos dados do seguimento tardio, considerou-se sempre o primeiro evento ocorrido e excluíam-se aqueles da fase hospitalar.

Os óbitos foram classificados como cardíacos ou não cardíacos. Todas as mortes foram consideradas cardíacas, exceto quando uma causa não cardíaca inequívoca fosse identificada. As mortes súbitas também foram classificadas como óbitos cardíacos, a não ser nos casos em que havia algum diagnóstico de doença não cardíaca potencialmente letal.

O infarto do miocárdio (IM), logo após o procedimento (tipo 4a), foi definido como a elevação da CK-MB massa pelo menos $\geq 5$ vezes o valor do percentil 99 do limite superior do valor de referência.(109). Os infartos espontâneos na evolução foram aqueles identificados pela elevação de pelo menos duas vezes o valor do percentil 99 do limite superior do valor de referência dos marcadores de necrose. 
Reinfarto pós-ICP foi considerado quando houve elevação pós-SF de mais de $20 \%$ no valor da CKMB massa de base. Caso a CKMB massa não estivesse estável antes da ICP, este evento não pôde ser julgado.

Todos os infartos foram classificados como "Q" ou não "Q", com base nas alterações do eletrocardiograma, ou desconhecido (ausência de documentação).

A revascularização da lesão-alvo foi definida como qualquer nova intervenção percutânea ou cirúrgica, devido à recorrência da lesão, dentro do stent e/ou nas suas bordas ( $5 \mathrm{~mm}$ proximais ou distais às bordas do SF, na avaliação angiográfica).

\section{Trombose protética}

A trombose protética foi definida e classificada de acordo com os critérios do Academic Research Consortium (ARC). ${ }^{(110)}$ Casos incluídos anteriormente a estas definições foram revistos e uniformizados.

Considerando-se o grau de certeza do diagnóstico, foram divididas em:

a) Definitiva:

- sintomas de síndrome coronária aguda

- confirmação angiográfica ou anátomo-patológica da presença de trombo ao nível do stent 
b) Provável:

- morte súbita até 30 dias da intervenção coronária percutânea

- infarto no território miocárdico tratado, ainda que sem confirmação angiográfica da oclusão do stent

c) Possível:

- morte súbita decorridos mais que 30 dias do procedimento.

De acordo com o momento de ocorrência da trombose, esta foi classificada como:
a) Aguda: $\leq 24$ horas
b) Sub aguda: $>24$ horas até 30 dias
c) Tardia: $>30$ dias até 12 meses
d) Muito tardia: > 12 meses.

\subsubsection{Análise estatística}

As análises foram realizadas em dois momentos distintos: o primeiro, com os dados retrospectivos dos pacientes incluídos entre janeiro de 2007 e dezembro de 2012, e que serviram para o desenvolvimento do escore DESIRE em duas fases: hospitalar e evolutiva (tardia). A seguir, prospectivamente, o escore foi validado nos pacientes incluídos de janeiro de 2013 a dezembro de 2014. Também considerando-se as duas fases mencionadas. 
Para o presente estudo utilizamos o auxílio do programa estatístico R versão 3.3.1. ${ }^{(111)}$

\subsubsection{Análise descritiva}

As coortes B1 e B2 foram comparadas quanto às suas características clínicas e angiográficas pré-procedimento bem como, em relação aos aspectos técnicos da ICP e, também aos ECM imediatos e até 2 anos de seguimento. Para a análise descritiva, as variáveis categóricas foram representadas por suas respectivas frequências e porcentagens, e comparadas pelo teste Qui-quadrado. As variáveis contínuas foram descritas como médias e desvios padrão e testadas pelo teste t-Student.

\subsubsection{Desenvolvimento do escore DESIRE}

Inicialmente, as associações das variáveis pré-ICP com os ECM foram testadas, considerando o nível de significância de 5\% para estes testes. Algumas variáveis, como por exemplo a idade, foram inseridas no modelo independentemente do resultado da regressão logística simples.

As variáveis, que se mostraram significativamente relacionadas à ocorrência de ECM, foram incluídas na análise de regressão logística múltipla. Devido à identificação de diferentes variáveis com efeito prognóstico nas fases hospitalar e tardia, decidimos por dois modelos de predição. Para a fase hospitalar, propusemos o modelo de regressão 
logística ${ }^{(115)}$ e, para a fase tardia, o modelo de riscos proporcionais de Cox ${ }^{(112)}$, estimando o tempo até o primeiro evento.

A seleção das variáveis para a escolha do modelo final, por sua vez, foi realizada pelo método stepwise (backward), segundo critério de Akaike nas duas fases, hospitalar e tardia. (113)

A escolha da pontuação simplificada para cada variável selecionada foi baseada numa medida proporcional às respectivas razões de chance (odds ratio) e taxas de risco (hazard ratios), estimadas pelos modelos. As probabilidades estimadas de ECM, segundo o modelo logístico e aproximadas pela pontuação (modelo simplificado), apresentaram relação cúbica. Para descrever a qualidade da correlação entre as estimativas do modelo e a pontuação ajustaram-se modelos lineares com relação cúbica e destacou-se o coeficiente de determinação $\mathrm{R}^{2}$.

A determinação das faixas de risco foi arbitrária, a partir de uma avaliação da distribuição dos riscos. Definiu-se como baixo risco os pacientes que apresentavam probabilidade de ocorrência de ECM até a taxas de ECM que fosse encontrada na população de desenvolvimento do escore, para a fase correspondente, sendo que para o escore tardio consideramos até dois anos de seguimento. A partir daí, classificou-se como de moderado risco aqueles que apresentassem a média até dobro da probabilidade e, acima do dobro, alto risco.

A avaliação da acurácia do modelo nos próprios dados foi medida pela área sob a curva ROC, para ECM hospitalares. Para os ECM tardios, usamos os modelos de sobrevivência de Haegerty e Zheng. ${ }^{(114)}$ 


\subsubsection{Validação do escore DESIRE}

A validação do escore foi realizada na coorte B2, pela análise da curva ROC para os desfechos em ambos os períodos, hospitalar e tardio.

Além disso, utilizando os três cortes propostos para a identificação de estratos de risco na construção dos escores, calculou-se a taxa de ECM caso a caso comparando-a com a probabilidade estimada, por meio do teste de aderência (Qui-quadrado). 
4 RESULTADOS 


\subsection{Casuística}

Foram analisados 4.061 pacientes do Registro DESIRE, que constituem a coorte B (figuras 8 e 9) e que representam $57,4 \%$ de todos os casos incluídos neste banco de dados, até dezembro de 2016. Essa população foi dividida em dois grupos para: 1) desenvolvimento do escore DESIRE ( $n=2.863 P$-coorte B1) e 2) validação do escore DESIRE ( $n=1.198 P$ coorte B2). (Figura 8)

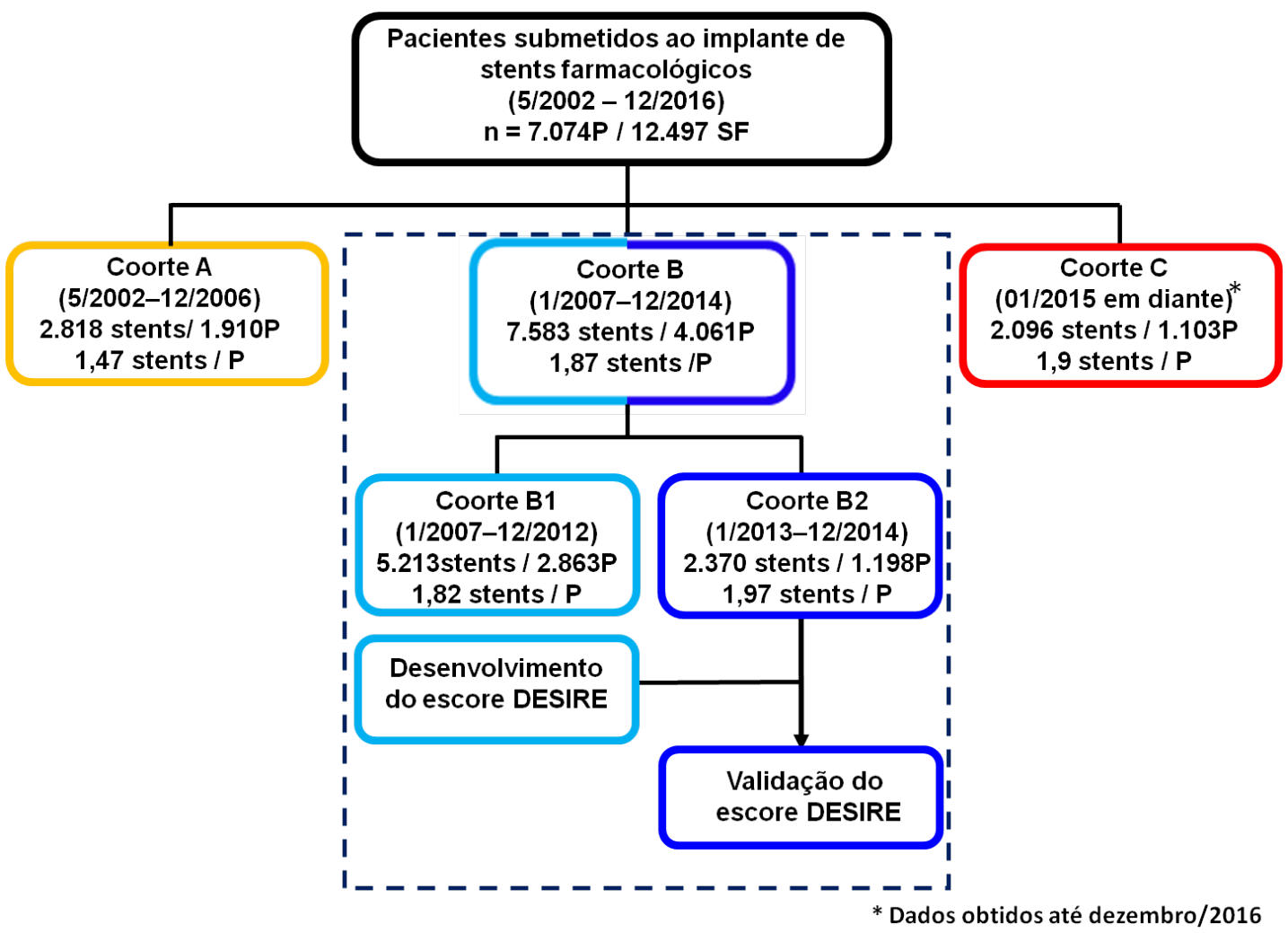

Figura 8 - Fluxograma de inclusão de pacientes no Registro DESIRE. Em amarelo, a coorte A, na qual predomina o uso dos SF de primeira geração. Destaca-se em azul, a coorte B, casuística desta pesquisa. Em azul claro, a coorte B1, utilizada a para o desenvolvimento do escore, o qual foi aplicado e validado na coorte B2 (azul escuro) nas duas fases do seguimento clínico, hospitalar e tardia. Em vermelho a coorte C, na qual estão sendo inseridos os pacientes que se submeteram procedimentos após o final do recrutamento deste estudo. 


\subsection{Características clínicas}

As características demográficas e a apresentação clínica dos pacientes das coortes B, B1 e B2, incluindo a análise comparativa entre os dois subgrupos (B1 e B2), encontram-se nas tabelas 2 e 3.

Observaram-se diferenças estatisticamente significantes entre as coortes B1 e B2. B1 apresentou maior frequência de alguns fatores de risco e comorbidades, como: história familiar para DAC $(p=0,02)$, tabagismo atual $(p=0,01)$, insuficiência renal crônica $(p=0,03)$ e também de eventos e procedimentos cardiovasculares prévios: infarto do miocárdio $(<0,001), \operatorname{ICP}(p=0,004)$ e CRM $(p<0,001)$, quando comparada à coorte B2. Por outro lado, esta coorte, na qual o escore DESIRE foi validado, teve média das idades mais elevada $(p=0,02)$, e infarto do miocárdio na apresentação foi mais frequente, do que na $B 1(p=0,002)$. 
Tabela 2 - Características clínicas dos pacientes das coortes B, B1 e B2

\begin{tabular}{|c|c|c|c|c|}
\hline Variável Coorte & $\begin{array}{c}\text { B } \\
4061 P\end{array}$ & $\begin{array}{c}\text { B1 } \\
2863 P\end{array}$ & $\begin{array}{c}\text { B2 } \\
1198 P\end{array}$ & Valor $\mathrm{p}^{*}$ \\
\hline Idade, anos, média (DP) & $65,0(11,0)$ & $64,8(10,9)$ & $65,7(11,2)$ & $0,02^{\ddagger}$ \\
\hline Sexo masculino, $N(\%)$ & $\begin{array}{c}3176 \\
(78,2 \%)\end{array}$ & $\begin{array}{c}2238 \\
(78,2 \%)\end{array}$ & $\begin{array}{c}938 \\
(78,3 \%)\end{array}$ & $0,96^{\#}$ \\
\hline
\end{tabular}

Fatores de risco, $\mathbf{N}(\%)$

\begin{tabular}{lcccc}
\hline História familiar de DAC & 1172 & 858 & 314 & $0,02^{\#}$ \\
& $(28,8 \%)$ & $(30 \%)$ & $(26,2 \%)$ & \\
Diabete melito & 1338 & 916 & 422 & $0,05^{\#}$ \\
Hipertensão arterial & $(32,9 \%)$ & $(32,0 \%)$ & $(35,2 \%)$ & \\
& 3144 & 2218 & 926 & $0,93^{\#}$ \\
Dislipidemia & $(77,4 \%)$ & $(77,5 \%)$ & $(77,3 \%)$ & \\
& 2617 & 1848 & 769 & $0,85^{\#}$ \\
Tabagismo atual & $(64,4 \%)$ & $(64,5 \%)$ & $(64,2 \%)$ & \\
& $(321$ & 246 & 75 & $0,01^{\#}$ \\
Obesidade $^{\dagger}$ & $(7,9 \%)$ & $(8,6 \%)$ & $(6,2 \%)$ & \\
& $(1180$ & 840 & 340 & $0,81^{\#}$ \\
\hline
\end{tabular}

\section{Eventos e procedimentos cardiovasculares prévios, $\mathbf{N}(\%)$}

\begin{tabular}{lcccc}
\hline IM & 843 & 678 & 165 & $<0,001^{\#}$ \\
AVE & $(20,8 \%)$ & $(23,7 \%)$ & $(13,8 \%)$ & \\
& 79 & 63 & 16 & $0,09^{\#}$ \\
ICP & $(1,9 \%)$ & $(2,2 \%)$ & $(1,3 \%)$ & \\
& 948 & 704 & 244 & $0,004^{\#}$ \\
CRM & $(23,3 \%)$ & $(24,6 \%)$ & $(20,3 \%)$ & \\
& 908 & 686 & 222 & $<0,001^{\#}$ \\
\hline
\end{tabular}

\section{Comorbidades, $\mathbf{N}(\%)$}

\begin{tabular}{lcccc}
\hline Doença vascular periférica & 120 & 86 & 34 & $0,85^{\#}$ \\
& $(3,0 \%)$ & $(3,0 \%)$ & $(2,8 \%)$ & \\
Insuficiência renal $(\mathrm{Cr})^{\star *}$ & 266 & 203 & 63 & $0,04^{\#}$ \\
& $(6,6 \%)$ & $(7,1 \%)$ & $(5,2 \%)$ & \\
Insuficiência renal $(\mathrm{ClCr})^{\star * *}$ & 886 & 671 & 215 & $<0,001$ \\
\hline
\end{tabular}

$\mathrm{B}$ = coorte desta investigação; $\mathrm{B} 1$ = coorte de desenvolvimento do escore DESIRE; B2 = coorte de validação; $\mathrm{AVE}=$ acidente vascular encefálico; $\mathrm{CICr}=$ clearance de creatinina; $\mathrm{Cr}=$ creatinina; $\mathrm{CRM}=$ cirurgia de revascularização miocárdica; $\mathrm{DAC}=$ doença arterial coronária; $\mathrm{DP}=$ desvio padrão; ICP = intervenção coronária percutânea; $\mathrm{P}=$ pacientes; $\mathrm{N}=$ número de casos; IM = infarto do miocárdio; $\left(^{\dagger}\right)$ Definida pelo IMC $>30 \mathrm{~kg} / \mathrm{m}^{2}$; ${ }^{*}$ Definida pela creatinina $(\mathrm{Cr}) \geq 1,5 \mathrm{mg} / \mathrm{dl}$; ${ }^{* * *}$ Definida pelo clearance de creatinina $(\mathrm{ClCr})<60 \mathrm{~mL} / \mathrm{min}\left(^{*}\right)$ Comparação entre as coortes B1 e B2, $\left(^{\#}\right)$ Teste Qui-quadrado, $\left(^{\ddagger}\right)$ Teste t-Student 
Tabela 3 - Apresentação clínica dos pacientes das coortes B, B1 e B2

\begin{tabular}{lccc} 
& B & B1 & B2 \\
& $\mathbf{4} 061 \mathbf{P}$ & $\mathbf{2 8 6 3} \mathbf{P}$ & $\mathbf{1 1 9 8} \mathbf{P}$ \\
$\begin{array}{l}\text { Apresentação } \\
\text { clínica, N (\%) }\end{array}$ & & & \\
\hline & & & \\
Isquemia silenciosa & 1673 & 1184 & 489 \\
& $(41,2 \%)$ & $(41,4 \%)$ & $(40,9 \%)$ \\
& & & \\
Angina estável & 635 & 469 & 166 \\
& $(15,6 \%)$ & $(16,4 \%)$ & $(13,8 \%)$
\end{tabular}

Síndrome coronária aguda

0,002

Angina instável

$\begin{array}{ccc}1009 & 725 & 284 \\ (24,8 \%) & (25,3 \%) & (23,7 \%)\end{array}$

IM recente

744

$(18,3 \%)$

485

259

(até 30 dias)

$(16,9 \%)$

$(21,6 \%)$

$\mathrm{B}$ = coorte de estudo; $\mathrm{B} 1$ = desenvolvimento; $\mathrm{B} 2$ = validação; $\mathrm{IM}$ = infarto do miocárdio; $\mathrm{N}$ = número de casos; $P$ = pacientes; $\left({ }^{\#}\right)$ Comparação entre as coortes B1 e B2, teste Qui-quadrado

\subsection{Características angiográficas e aspectos técnicos}

\subsubsection{Dados angiográficos pré-ICP}

Nas tabelas 4, 5 e 6 estão descritas: a fração de ejeção do VE e também as principais características angiográficas das lesões tratadas. 
Tabela 4 - Características angiográficas dos pacientes das coortes B, B1 e B2

\begin{tabular}{|c|c|c|c|c|}
\hline Variável Coorte & $\begin{array}{c}\text { B } \\
4061 \mathrm{P}\end{array}$ & $\begin{array}{c}\text { B1 } \\
2863 P\end{array}$ & $\begin{array}{c}\text { B2 } \\
1198 P\end{array}$ & Valor $\mathbf{p}^{*}$ \\
\hline $\begin{array}{l}\text { Doença } \\
\text { multiarterial**, N (\%) }\end{array}$ & $\begin{array}{c}2640 / 4061 \\
(65,0 \%)\end{array}$ & $\begin{array}{c}1933 / 2863 \\
(67,5 \%)\end{array}$ & $\begin{array}{c}707 / 1198 \\
(59,0 \%)\end{array}$ & $<0,001$ \\
\hline Fração de ejeção, $N(\%)^{\ddagger}$ & $3925 \mathrm{P}$ & $2754 P$ & $1171 P$ & Valor $\mathbf{p}^{*}$ \\
\hline \multicolumn{5}{|l|}{$\begin{array}{l}\mathrm{FE} \geq 40 \% \\
\text { (normal ou discreto } \\
\text { comprometimento) } \\
\end{array}$} \\
\hline $\mathrm{FE} \geq 55 \%$ & $2994(76,3 \%)$ & $2019(73,3 \%)$ & $975(83,3 \%)$ & \\
\hline $40 \% \leq \mathrm{FE}<55 \%$ & $625(15,9 \%)$ & $496(18,0 \%)$ & $129(11,0 \%)$ & \\
\hline $\begin{array}{l}\mathrm{FE}<40 \% \\
\text { (moderado ou importante } \\
\text { comprometimento) }\end{array}$ & & & & $<0,001$ \\
\hline $30 \% \leq \mathrm{FE}<40 \%$ & $267(6,8 \%)$ & $206(7,5 \%)$ & $61(5,2 \%)$ & \\
\hline FE $<30 \%$ & $39(1,0 \%)$ & $33(1,2 \%)$ & $6(0,5 \%)$ & \\
\hline
\end{tabular}

$\mathrm{B}=$ coorte de estudo; $\mathrm{B} 1$ = desenvolvimento; $\mathrm{B} 2$ = validação; $\mathrm{N}=$ número de casos; $\mathrm{P}=$ pacientes; $\left({ }^{* *}\right)$ Presença de lesão $\geq 50 \%$ em mais de um vaso; $\left(^{\ddagger}\right)$ Avaliação feita pela angiografia quantitativa $\left({ }^{\#}\right)$ comparação entre as coortes B1 e B2, teste Qui-quadrado

Tabela 5 - Características angiográficas pré-procedimento das vasos-alvo das coortes B, B1 e B2

\begin{tabular}{lcccc}
\hline \multicolumn{1}{c}{ Coorte } & B & B1 & B2 & Valor $\mathbf{p}^{\#}$ \\
$\begin{array}{lcccc}\text { Lesões no } \\
\text { Vaso-alvo, N (\%) }\end{array}$ & $\mathbf{6 ~ 8 6 2 ~ L}$ & $\mathbf{4 7 6 3 \mathbf { L }}$ & $\mathbf{2 ~ 0 9 9 ~ \mathbf { ~ }}$ & \\
\hline Coronária direita & $1899(27,7 \%)$ & $1323(27,8 \%)$ & $57627,4 \%)$ & \\
Circunflexa & $1857(27,1 \%)$ & $1297(27,2 \%)$ & $560(26,7 \%)$ & \\
Descendente anterior & $2658(38,8 \%)$ & $1823(38,2 \%)$ & $835(39,8 \%)$ & 0,003 \\
TCE não protegido & $113(1,6 \%)$ & $66(1,4 \%)$ & $47(2,2 \%)$ & \\
Ponte de safena & $314(4,6 \%)$ & $242(5,1 \%)$ & $72(3,4 \%)$ & \\
Enxerto arterial & $21(0,3 \%)$ & $12(0,3 \%)$ & $9(0,4 \%)$ &
\end{tabular}

$\mathrm{B}$ = coorte de estudo; $\mathrm{B} 1$ = desenvolvimento; $\mathrm{B} 2$ = validação; Considerando a presença de pelo menos uma lesão com a característica de risco; $\left({ }^{\#}\right)$ Comparação entre as coortes B1 e B2, teste Quiquadrado; $\mathrm{L}=$ lesões-alvo; $\mathrm{N}=$ número de casos; $\mathrm{P}=$ pacientes; $\mathrm{V}=$ vasos-alvo; $\mathrm{TCE}=$ tronco da coronária esquerda. 
Tabela 6 - Características angiográficas pré-procedimento das lesões-alvo das coortes B, B1 e B2

\begin{tabular}{|c|c|c|c|c|}
\hline $\begin{array}{l}\text { Características } \\
\text { da lesão-alvo, } \mathrm{N}(\%)\end{array}$ & $\begin{array}{c}\text { B } \\
6862 \mathrm{~L}\end{array}$ & $\begin{array}{c}\text { B1 } \\
4763 \mathrm{~L}\end{array}$ & $\begin{array}{c}\text { B2 } \\
2099 \text { L }\end{array}$ & $\begin{array}{c}\text { Valor } \\
\mathbf{p}^{\#}\end{array}$ \\
\hline Reestenótica & $\begin{array}{c}206 \\
(3,0 \%)\end{array}$ & $\begin{array}{c}149 \\
(3,1 \%)\end{array}$ & $\begin{array}{c}57 \\
(2,7 \%)\end{array}$ & 0,39 \\
\hline Ostial & $\begin{array}{c}364 \\
(5,3 \%)\end{array}$ & $\begin{array}{c}235 \\
(4,9 \%)\end{array}$ & $\begin{array}{c}129 \\
(6,1 \%)\end{array}$ & 0,04 \\
\hline Em bifurcação§ & $\begin{array}{c}628 \\
(9,1 \%)\end{array}$ & $\begin{array}{c}395 \\
(8,3 \%)\end{array}$ & $\begin{array}{c}233 \\
(11,1 \%)\end{array}$ & $<0,001$ \\
\hline Oclusão crônica & $\begin{array}{c}77 \\
(1,1 \%)\end{array}$ & $\begin{array}{c}41 \\
(0,9 \%)\end{array}$ & $\begin{array}{c}36 \\
(1,7 \%)\end{array}$ & 0,003 \\
\hline Com Trombo & $\begin{array}{c}182 \\
(2,7 \%)\end{array}$ & $\begin{array}{c}149 \\
(3,1 \%)\end{array}$ & $\begin{array}{c}33 \\
(1,6 \%)\end{array}$ & $<0,001$ \\
\hline
\end{tabular}

Calcificação

\begin{tabular}{lcccc}
\hline Ausente ou discreta & 4921 & 3545 & 1376 & \\
& $(71,7 \%)$ & $(74,4 \%)$ & $(65,6 \%)$ & \\
Moderada & 1629 & 1075 & 554 & $<0,001$ \\
& $(23,7 \%)$ & $(22,6 \%)$ & $(26,4 \%)$ & \\
Intensa & 312 & 143 & 169 & \\
& $(4,6 \%)$ & $(3,0 \%)$ & $(8,0 \%)$ & \\
\hline
\end{tabular}

Classificação da lesão ${ }^{\circ}$

$\begin{array}{lcccc}\text { A } & 356 & 350 & 6 & \\ & (5,2 \%) & (7,3 \%) & (0,3 \%) & \\ \text { B1 } & 1940 & 1364 & 576 & \\ & (28,3 \%) & (28,6 \%) & (27,4 \%) & <0,001 \\ \text { B2 } & 1013 & & & \\ & (14,7 \%) & (15,9 \%) & (12,2 \%) & \\ & & & & \\ \text { C } & 3553 & 2292 & 1261 & \\ & (51,8 \%) & (48,2 \%) & (60,1 \%) & \end{array}$

$\mathrm{B}$ = coorte de estudo; $\mathrm{B} 1$ = desenvolvimento; $\mathrm{B} 2$ = validação; Considerando a presença de pelo menos uma lesão com a característica de risco. $\left({ }^{\S}\right)$ Definida pela presença de estenose $\geq 50 \%$ no vaso principal e no vaso lateral, sendo este de diâmetro $\geq 2,0 \mathrm{~mm}$ (estimativa visual); $\left({ }^{\sigma}\right)$ De acordo com a classificação do American College of Cardiology/American Heart Association ${ }^{(81)}$; $L$ = lesõesalvo; $\mathrm{N}=$ número de casos; $\left(^{\#}\right)$ Comparação entre as coortes B1 e B2, teste Qui-quadrado 
As coortes B1 e B2 mostraram-se estatisticamente diferentes quanto à maioria das características angiográficas. Observou-se que, em B1, os pacientes apresentaram, com mais frequência: doença multiarterial $(p<0,001)$, disfunção ventricular esquerda $(p<0,001)$, ponte de safena como vaso-alvo $(p=0,003)$ e trombo na lesão-alvo $(p<0,001)$. Por outro lado, na coorte B2 foram mais prevalentes: as lesões ostiais $(p=0,04)$, em bifurcações $(p<0,001)$, as oclusões crônicas $(p=0,003)$, as lesões calcificadas $(p<0,001)$ e aquelas do tipo B2 e $C(p<0,001)$.

Os dados da angiografia coronária quantitativa pré-ICP estão listados na tabela 7 .

Tabela 7 - Dados da angiografia coronária quantitativa pré-ICP das coortes B, B1 e B2

\begin{tabular}{|c|c|c|c|c|}
\hline$\times$ & $\begin{array}{c}\text { B } \\
6862 \mathrm{~L} \\
\text { média (DP) }\end{array}$ & $\begin{array}{c}\text { B1 } \\
4763 \mathrm{~L} \\
\text { média (DP) }\end{array}$ & $\begin{array}{c}\text { B2 } \\
2099 \text { L } \\
\text { média (DP) }\end{array}$ & $\begin{array}{c}\text { Valor } \\
\mathbf{p}^{\ddagger}\end{array}$ \\
\hline $\begin{array}{l}\text { Diâmetro de referência } \\
\text { interpolado, mm }\end{array}$ & $2,71(0,52)$ & $2,75(0,51)$ & $2,63(0,51)$ & $<0,001$ \\
\hline Extensão da lesão, mm & $19,21(10,06)$ & $18,62(9,06)$ & $20,62(12,02)$ & $<0,001$ \\
\hline $\begin{array}{l}\text { Diâmetro mínimo da luz, } \\
\text { mm }\end{array}$ & $0,89(0,33)$ & $0,9(0,32)$ & $0,87(0,33)$ & 0,003 \\
\hline Grau da lesão, \% & $68,03(9,79)$ & $67,91(9,40)$ & $68,33(10,60)$ & 0,11 \\
\hline
\end{tabular}




\subsubsection{Aspectos técnicos}

Nos 4.061 pacientes incluídos, 6.862 lesões foram tratadas (1,7 lesões / paciente) em 5.715 vasos, com 7.583 SF implantados (1,9 SF / paciente). Os dados dos procedimentos, bem como os tipos de SF usados estão relacionados na tabela 8 . Na coorte B2, foram utilizados exclusivamente os SF de segunda e terceira gerações $(p<0,001)$.

$\mathrm{Na}$ coorte B1 as médias das pressões máximas de insuflação foram mais baixas $(p<0,001)$ e praticou-se mais frequentemente a pós-dilatação $(p=0,03)$. Na B2, a média dos diâmetros dos stents foi menor $(p<0,001)$ e a média das extensões dos mesmos foi maior $(p<0,001)$, assim como a razão stents / paciente foi também maior $(p<0,001)$. (Tabela 8) 
Tabela 8 - Características dos procedimentos das coortes B, B1 e B2

\begin{tabular}{|c|c|c|c|c|}
\hline \begin{tabular}{|lll} 
Variável Coorte & C \\
\end{tabular} & $\begin{array}{r}\text { B } \\
7583 \text { SF }\end{array}$ & $\begin{array}{c}\text { B1 } \\
5213 \text { SF }\end{array}$ & $\begin{array}{c}\text { B2 } \\
2370 \mathrm{SF}\end{array}$ & Valor $\mathrm{p}^{*}$ \\
\hline \multicolumn{5}{|c|}{ Características do procedimento } \\
\hline Pré-dilatação, N (\%) & $3874(51,1 \%)$ & $2675(51,3 \%)$ & $1199(50,6 \%)$ & $0,57^{\#}$ \\
\hline $\begin{array}{l}\text { Diâmetro do stent, mm, } \\
\text { média (DP) }\end{array}$ & $2,86(0,65)$ & $2,88(0,49)$ & $2,82(0,91)$ & $<0,001^{\ddagger}$ \\
\hline $\begin{array}{l}\text { Extensão do stent, mm, } \\
\text { média (DP) }\end{array}$ & $21,19(7,04)$ & $20,68(6,68)$ & $22,30(7,65)$ & $<0,001^{\ddagger}$ \\
\hline $\begin{array}{l}\text { Razão stent/ paciente, } \\
\text { média (DP) }\end{array}$ & $1,9(1,02)$ & $1,9(0,97)$ & $2,0(1,14)$ & $<0,001^{\ddagger}$ \\
\hline $\begin{array}{l}\text { Pressão máxima de } \\
\text { insuflação, atm, média (DP) }\end{array}$ & $10,81(2,81)$ & $10,42(2,63)$ & $11,68(2,99)$ & $<0,001^{1}$ \\
\hline Pós-dilatação, N (\%) & $5351(70,6 \%)$ & $3639(69,8 \%)$ & $1712(72,3 \%)$ & $0,03^{\#}$ \\
\hline
\end{tabular}

\begin{tabular}{|c|c|c|c|c|}
\hline Tipos de SF implantados & & & & \\
\hline \multicolumn{5}{|l|}{$1^{a}$ geração } \\
\hline Cypher $^{\circledR}$ & $2669(35,2 \%)$ & $2669(51,2 \%)$ & $0(0,0 \%)$ & \\
\hline Taxus $^{\circledR}$ & $117(1,5 \%)$ & $116(2,2 \%)$ & $1(0,04 \%)$ & \\
\hline \multicolumn{5}{|l|}{$2^{a}$ geração } \\
\hline Endeavor $^{\circledR}$ & $108(1,4 \%)$ & $108(2,1 \%)$ & $0(0,0 \%)$ & \\
\hline Xience $^{\circledR} /$ Promus $^{\circledR}$ & $2672(35,3 \%)$ & $1438(27,6 \%)$ & $1234(52,1 \%)$ & $<0,001^{\ddagger}$ \\
\hline Resolute $^{\circledR}$ & $1455(19,2 \%)$ & $667(12,8 \%)$ & $788(33,2 \%)$ & \\
\hline \multicolumn{5}{|l|}{$3^{\mathrm{a}}$ geração } \\
\hline Biomatrix $^{\circledR}$ & $454(6,0 \%)$ & $215(4,1 \%)$ & $239(10,1 \%)$ & \\
\hline Synergy ${ }^{\circledR}$ & $108(1,4 \%)$ & $0(0,0 \%)$ & $108(4,6 \%)$ & \\
\hline
\end{tabular}

Resultado do
procedimento

procedimento

Fluxo TIMI 3, N (\%)

$7563(99,8 \%) \quad 5196(99,7 \%)$

$2367(99,9 \%)$

$0,34^{\#}$

Sucesso angiográfico, N (\%) $7558(99,7 \%) \quad 5196(99,7 \%) \quad 2362(99,7 \%) \quad 0,99^{\#}$

$\mathrm{B}$ = coorte de estudo; $\mathrm{B} 1$ = desenvolvimento; $\mathrm{B} 2$ = validação; $\mathrm{DP}$ = desvio padrão; $\mathrm{N}$ = número de casos; SF = stents farmacológicos; TIMI = classificação do fluxo no vaso-alvo segundo o grupo TIMI; $\left({ }^{*}\right)$ Comparação entre as coortes B1 e B2, $\left(^{\#}\right)$ Teste Qui-quadrado, $\left(^{\ddagger}\right)$ Teste t-Student 
Houve insucesso do procedimento em 12 pacientes $(12 / 4.061=0,3 \%)$, nos quais foram utilizados 25 SF. Este resultado ocorreu em 10 casos $(0,35 \%)$ na coorte B1 e em $2(0,17 \%)$ na B2 ( $p=0,12)$. A calcificação moderada ou intensa foi a principal causa de insucesso técnico em 7 pacientes, impedindo o avanço do dispositivo até a lesão-alvo ou então determinando a inadequada expansão do mesmo. As demais causas foram: 2 casos com perfurações coronárias; 1 com fenômeno de noreflow em uma ICP em ponte de safena e 1 com falha na recanalização de oclusão crônica.

$\mathrm{Na}$ tabela 9 estão listados os dados da angiografia coronária quantitativa pós-ICP.

\begin{tabular}{|c|c|c|c|c|}
\hline Variável & $\begin{array}{c}\text { B } \\
6862 \mathrm{~L} \\
\text { média (DP) }\end{array}$ & $\begin{array}{c}\text { B1 } \\
4763 \mathrm{~L} \\
\text { média (DP) }\end{array}$ & $\begin{array}{c}\text { B2 } \\
2099 \mathrm{~L} \\
\text { média (DP) }\end{array}$ & $\begin{array}{c}\text { Valor } \\
\mathbf{p}^{\#}\end{array}$ \\
\hline $\begin{array}{l}\text { Diâmetro de referência } \\
\text { interpolado, } \mathrm{mm}\end{array}$ & $2,73(0,51)$ & $2,76(0,53)$ & $2,65(0,53)$ & $<0,001$ \\
\hline $\begin{array}{l}\text { Diâmetro mínimo da } \\
\text { luz, mm }\end{array}$ & $2,82(0,52)$ & $2,84(0,51)$ & $2,78(0,52)$ & $<0,001$ \\
\hline Estenose residual, \% & $2,32(2,97)$ & $2,68(3,36)$ & $1,52(1,51)$ & $<0,001$ \\
\hline Ganho imediato, $\mathrm{mm}$ & $1,94(0,41)$ & $1,95(0,42)$ & $1,91(0,42)$ & $<0,001$ \\
\hline
\end{tabular}




\subsection{Dados evolutivos}

As médias dos tempos de internação nas coortes B, B1 e B2 foram de: 2,7 dias ( $\mathrm{DP}=3,9), 2,5$ dias $(\mathrm{DP}=3,1)$ e 3,2 dias $(\mathrm{DP}=5,4)$, respectivamente. Neste aspecto, a diferença encontrada entre as coortes B1 e B2 foi significante $(p<0,001)$.

O acompanhamento clínico atualizado foi alcançado em $96,5 \%$ dos casos na coorte B (3.922 P / 4.061 P); em 96,4\% (2.760 P / $2.863 \mathrm{P})$ na coorte B1 e em 96,8\% (1.162 P / $1.198 \mathrm{P})$ na coorte B2 $(p<0,001)$. As médias dos tempos de seguimento foi de 4,4 anos (DP $=2,3$ ) na coorte $B$, de 5,3 anos (DP $=2,1)$ na $B 1$ e de 2,1 anos na $B 2(D P=0,6)$. Não foi possível obter informações do seguimento clínico em 0,54\% dos pacientes (22 casos). 


\subsubsection{Eventos cardíacos maiores}

Na fase hospitalar, os ECM ocorreram de forma similar nas coortes B1 e B2. Ocorreram $14(0,34 \%)$ óbitos antes da alta hospitalar, sendo $10(0,35 \%)$ na coorte B1 e $4(0,34 \%)$ na B2. Destes, 10/14 $(71,4 \%)$ foram óbitos cardíacos.

O evento predominante antes da alta foi o infarto do miocárdio. Quando se considerou, para a definição do Infarto tipo 4a, a elevação da CK-MB massa $\geq 3$ vezes (o percentil 99 do limite superior do valor de referência), este evento ocorreu em 335 pacientes (8,3\%); 25 vezes em 157 $\operatorname{casos}(3,9 \%)$.

A taxa de ECM hospitalares foi de 4,1\% (166 pacientes), considerando para o diagnóstico do infarto do miocárdio tipo $4 a$, valores de CK-MB massa $\geq 5$ vezes o percentil 99 .

Na tabela 10, estão listados os eventos cardíacos maiores hospitalares nas coortes B, B1 e B2. Destaque-se que consideramos, para caracterizar todos os infartos do miocárdio periprocedimento, as elevações $\geq 5$ vezes o percentil 99, de acordo com as definições já mencionadas. 
Tabela 10 - Distribuição dos eventos cardíacos maiores nas coortes B, B1 e B2 na fase hospitalar

\begin{tabular}{|c|c|c|c|c|}
\hline ECM, $N(\%)$ & $\begin{array}{c}B^{¥} \\
4061 P\end{array}$ & $\begin{array}{c}B 1^{\phi} \\
2863 P\end{array}$ & $\begin{array}{c}B 2 \\
1198 P\end{array}$ & Valor $p^{\#}$ \\
\hline Óbito cardíaco & $10(0,25 \%)$ & $7(0,24 \%)$ & $3(0,25 \%)$ & 0,99 \\
\hline \multicolumn{5}{|l|}{$\begin{array}{l}\text { Infarto do } \\
\text { miocárdio }\end{array}$} \\
\hline$C K M B \geq 3 x$ & $335(8,3 \%)$ & $231(8,1 \%)$ & $104(8,7 \%)$ & 0,56 \\
\hline$C K M B \geq 5 x$ & $157(3,9 \%)$ & $104(3,6 \%)$ & $53(4,4 \%)$ & 0,27 \\
\hline $\begin{array}{l}\text { Revascularização } \\
\text { da lesão-alvo }\end{array}$ & $0(0,0)$ & $0(0,0)$ & $0(0,0)$ & - \\
\hline $\begin{array}{l}\text { ECM combinados }{ }^{\&} \\
(\mathrm{CKMB} \geq 5 \mathrm{x})\end{array}$ & $166(4,1 \%)$ & $110(3,8 \%)$ & $56(4,7 \%)$ & 0,26 \\
\hline \multicolumn{5}{|c|}{$\begin{array}{l}\mathrm{B}=\text { coorte de estudo; } \mathrm{B} 1=\text { desenvolvimento; } \mathrm{B} 2=\text { validação; }\left({ }^{\star}\right) \mathrm{ECM}=\text { eventos cardíacos } \\
\text { maiores; } \mathrm{N}=\text { número de casos; } \mathrm{P}=\text { pacientes; } \geq 3 \mathrm{x} \text { ou } \geq 5 \mathrm{x}=\geq 3 \mathrm{vezes} \text { ou } \geq 5 \text { vezes o } \\
\text { percentil } 99 \text { do limite superior do valor de referência; }\left({ }^{*}\right) \text { na coorte } \mathrm{B}, 1 \text { paciente apresentou } \\
\text { óbito cardíaco e infarto do miocárdio. Este paciente pertence à coorte B1 }\left({ }^{\phi}\right) \text {. } \\
\left(^{\#}\right) \text { Comparação entre as coortes B1 e B2, teste Qui-quadrado }\end{array}$} \\
\hline
\end{tabular}

$\mathrm{Na}$ tabela 11, constam as taxas de ECM, excluindo-se os ECM ocorridos na fase hospitalar. Para efeito de comparação entre as coortes B1 e B2, analisamos os resultados de 2 anos de seguimento. 
Tabela 11 - Distribuição dos eventos cardíacos maiores não cumulativos das coortes B, B1 e B2 na fase tardia (até 2 anos de seguimento)

\begin{tabular}{|c|c|c|c|c|}
\hline $\mathrm{ECM}, \mathrm{N}(\%) \quad$ Coorte & $\begin{array}{c}B^{*} \\
3922 \mathrm{P}\end{array}$ & $\begin{array}{c}B 1^{\phi} \\
2760 P\end{array}$ & $\begin{array}{l}\mathrm{B2}^{£} \\
1162 \mathrm{P}\end{array}$ & Valor \\
\hline Óbito cardíaco & $79(2,01 \%)$ & $57(2,0 \%)$ & $26(2,2 \%)$ & 0,24 \\
\hline Infarto do miocárdio & $68(1,7 \%)$ & $46(1,6 \%)$ & $28(2,4 \%)$ & 1 \\
\hline RLA & $115(2,9 \%)$ & $76(2,7 \%)$ & $51(4,3 \%)$ & 0,39 \\
\hline ECM combinados* & $247(6,3 \%)$ & $173(6,3 \%)$ & $91(7,8 \%)$ & 0,13 \\
\hline
\end{tabular}

$\mathrm{B}$ = coorte de estudo; $\mathrm{B} 1$ = desenvolvimento; $\mathrm{B} 2$ = validação; $\mathrm{ECM}=$ eventos cardíacos maiores; $N$ = número de casos; $P$ = pacientes; $R L A$ = revascularização da lesão-alvo;

$\left(^{\#}\right)$ Comparação entre as coortes B1 e B2, teste Qui-quadrado

\subsubsection{Trombose protética}

As taxas de trombose protética nas coortes B, B1 e B2 encontram-se na tabela 12, onde estão discriminadas por tempo de ocorrência.

Tabela 12 - Incidência acumulada de trombose protética nas coortes B, B1 e B2 até 2 anos de seguimento

\begin{tabular}{|c|c|c|c|c|}
\hline $\begin{array}{l}\text { Trombose Coorte } \\
\text { Protética, N (\%) }\end{array}$ & $\begin{array}{c}\text { B } \\
4061 \mathrm{P}\end{array}$ & $\begin{array}{c}\text { B1 } \\
2863 \text { P }\end{array}$ & $\begin{array}{c}B 2 \\
1198 \mathrm{P}\end{array}$ & Valor $\mathbf{p}^{\#}$ \\
\hline Até 30 dias & $11(0,27 \%)$ & $6(0,21 \%)$ & $5(0,42 \%)$ & 0,402 \\
\hline até 1 ano & $28(0,69 \%)$ & $19(0,67 \%)$ & $9(0,76 \%)$ & 0,912 \\
\hline até 2 anos & $34(0,84 \%)$ & $24(0,84 \%)$ & $10(0,83 \%)$ & 0,864 \\
\hline
\end{tabular}

$\mathrm{B}=$ coorte de estudo; $\mathrm{B} 1$ = desenvolvimento; $\mathrm{B} 2$ = validação; $\mathrm{N}=$ número de casos; $\mathrm{P}=$ pacientes; $\left(^{\#}\right)$ comparação entre as coortes B1 e B2, teste Qui-quadrado 
A tabela 13 mostra a classificação e a distribuição temporal dos 34 casos que apresentaram trombose protética na coorte B $(0,84 \%)$, até 2 anos de seguimento, sendo 24 pacientes $(0,84 \%)$ na B1 e $10(0,83 \%)$ na B2.

Quanto ao grau de certeza, em B1 foram: $12(0,42 \%)$ os casos com trombose definitiva, 1 com trombose provável $(0,03 \%)$ e 11 com trombose possível $(0,38 \%)$. Na B2, ocorreram; 5 casos com trombose definitiva (0,42\%), 1 com provável $(0,08 \%)$ e 4 com possível $(0,34 \%)$.

Com relação ao tempo de ocorrência; 1 (0,03\%) paciente evoluiu com trombose aguda, $11(0,27 \%)$ com trombose subaguda, $16(0,39 \%)$ com trombose tardia e $6(0,15 \%)$ com tromboses muito tardia.

Tabela 13 - Classificação e distribuição temporal das 34 tromboses protéticas nas coortes $\mathrm{B}, \mathrm{B} 1$ e B2, até 2 anos de seguimento, de acordo com Academic Research Consortium (ARC) $)^{(111)}$

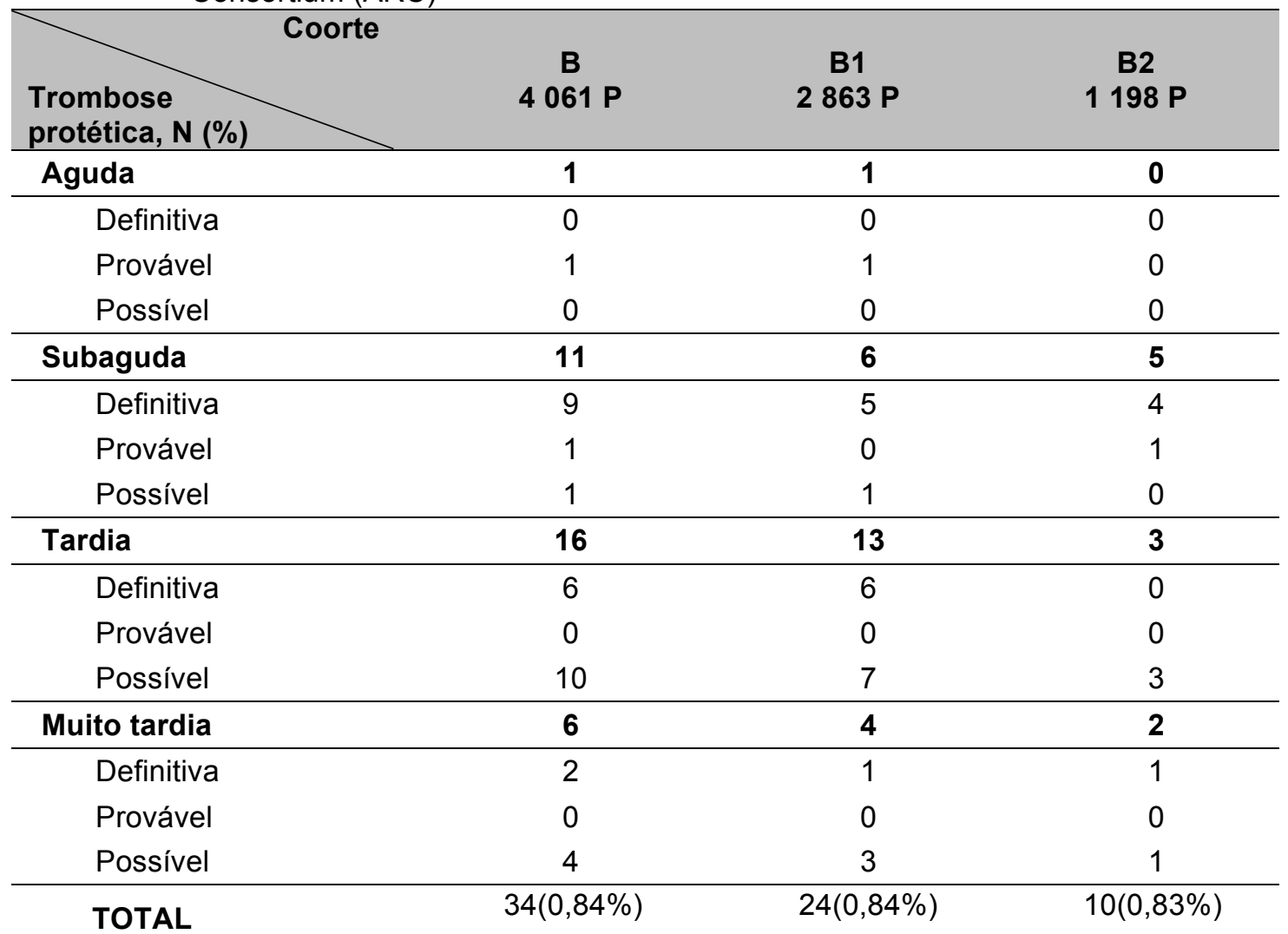

$\mathrm{B}=$ coorte de estudo; $\mathrm{B} 1$ = desenvolvimento; $\mathrm{B} 2$ = validação; $\mathrm{N}=$ número de casos; $\mathrm{P}=$ pacientes 
4.5 Desenvolvimento e validação do escore DESIRE hospitalar

\subsubsection{Desenvolvimento do escore DESIRE hospitalar}

O resultado da regressão logística univariada, utilizada para testar as associações das variáveis clínicas e angiográficas pré-ICP com os ECM, está descrito nas tabelas de 14 a 20. 
Tabela 14 - Resultados da análise da associação da idade, do gênero e dos fatores de risco com os ECM hospitalares pela regressão logística univariada (desenvolvimento do escore DESIRE hospitalar)

\begin{tabular}{|c|c|c|c|c|c|c|}
\hline \multirow{2}{*}{ Variável } & \multirow{2}{*}{$\begin{array}{c}2863 \mathrm{P} \\
\mathrm{N}(\%) \\
\end{array}$} & \multicolumn{2}{|c|}{$110(3,8) \mathrm{ECM}$} & \multirow{2}{*}{ OR } & \multirow{2}{*}{$\begin{array}{c}\text { IC } \\
(95 \%)\end{array}$} & \multirow{2}{*}{$\begin{array}{c}\text { Valor } \\
\text { p }\end{array}$} \\
\hline & & $\mathbf{N}$ & $\%$ & & & \\
\hline \multicolumn{7}{|l|}{ Idade } \\
\hline $\begin{array}{c}\text { Média }=65,1 \text { anos } \\
(\mathrm{DP}=11,0)\end{array}$ & - & - & - & 1,05 & $1,03-1,07$ & $<0,01$ \\
\hline$<70$ anos & $1868(65,2)$ & 53 & 2,8 & 1 & 1 & $<0,01$ \\
\hline$\geq 70$ anos & $995(34,8)$ & 57 & 5,7 & 2,08 & $1,42-3,06$ & \\
\hline \multicolumn{7}{|l|}{ Sexo } \\
\hline Feminino & $625(21,8)$ & 27 & 4,3 & 1 & 1 & 0,56 \\
\hline Masculino & $2238(78,2)$ & 83 & 3,7 & 0,85 & $0,55-1,35$ & \\
\hline
\end{tabular}

\section{Fatores de risco}

História familiar de DAC

Não

Sim

$\begin{array}{cccccc}2005(70) & 86 & 4,3 & 1 & 1 & 0,07 \\ 858(30) & 24 & 2,8 & 0,65 & 0,4-1,01 & \end{array}$

Diabete melito

Não

Sim

sem medicação

com medicação oral

em uso de insulina

Hipertensão arterial

Não

Sim

Dislipidemia

Não

Sim

Tabagismo

Nunca

Ex-tabagista

Fumante atual

Obesidade $^{\dagger}$

Não

Sim

$\begin{array}{ccccc}1947(68) & 71 & 3,6 & 1 & 1 \\ 916(32) & 39 & 4,3 & 1,18 & 0,78-1,75 \\ 49(1,7) & 2 & 4,1 & 1,21 & 0,18-4,01 \\ 732(25,6) & 30 & 4,1 & 1,13 & 0,72-1,73 \\ 135(4,7) & 7 & 5,2 & 1,47 & 0,6-3,06\end{array}$

$\begin{array}{ccccc}2041(71,3) & 84 & 4,1 & 1 & 1 \\ 576(20,1) & 20 & 3,5 & 0,84 & 0,5-1,36 \\ 246(8,6) & 6 & 2,4 & 0,60 & 0,23-1,27\end{array}$


Tabela 15 - Resultados da análise da associação dos eventos e procedimentos cardiovasculares prévios com os ECM hospitalares pela regressão logística univariada (desenvolvimento do escore DESIRE hospitalar)

\begin{tabular}{lrrrrrr}
\multicolumn{1}{c}{ Resultados } & $2863 \mathrm{P}$ & $110(3,8) \mathrm{ECM}$ & OR & $\begin{array}{c}\text { IC } \\
(95 \%)\end{array}$ & $\begin{array}{c}\text { Valor } \\
\mathrm{p}\end{array}$ \\
\cline { 2 - 5 } Variável & $\mathrm{N}(\%)$ & $\mathrm{N}$ & $\%$ & & & \\
\hline
\end{tabular}

Eventos e procedimentos cardiovasculares prévios

IM

$\begin{array}{ccccccc}\text { Não } & 2185(76,3) & 88 & 4 & 1 & & 0,42 \\ \text { Sim } & 678(23,7) & 22 & 3,2 & 0,8 & 0,49-1,27 & \end{array}$

AVE

Não

Sim

ICP

Não

$\operatorname{Sim}$

CRM

Não

Sim

\section{Comorbidades}

Doença vascular periférica

$\begin{array}{cccccc}\text { Não } & 2777(97) & 102 & 3,7 & 1 & 1 \\ \text { Sim } & 86(3) & 8 & 9,3 & 2,73 & 1,18-5,5\end{array}$

Insuficiência renal $(\mathrm{Cr})^{\star *}$

$\begin{array}{lcccccc}\text { Não } & 2656(92,9) & 94 & 3,5 & 1 & 1 & <0,01 \\ \text { Sim } & 203(7,1) & 16 & 7,9 & 2,35 & 1,31-3,97 & \\ \text { ciência Renal (ClCr) } & & & & & & \\ & 2122(76,0) & 68 & 3,2 & 1 & 1 & <0,01 \\ & 671(24,0) & 41 & 6,1 & 1,97 & 1,31-2,92 & \end{array}$

AVE = acidente vascular encefálico; $\mathrm{B}=$ coorte de estudo; $\mathrm{B} 1$ = desenvolvimento; $\mathrm{B} 2=$ validação; $\mathrm{ClCr}=$ clearance de creatinina; $\mathrm{Cr}=$ creatinina; $\mathrm{CRM}=$ cirurgia de revascularização miocárdica; $\mathrm{ECM}$ = eventos cardíacos maiores; IC = intervalo de confiança; ICP = intervenção coronária percutânea; IM = infarto do miocárdio; $\mathrm{OR}=$ odds ratio, razão de chances; $\mathrm{P}=$ pacientes; $\mathrm{N}=$ número de casos ${ }^{* *}$ Definida pela Creatinina $\geq 1,5 \mathrm{mg} / \mathrm{dl} ;{ }^{* * *}$ Definida pelo clearance de creatinina $<60 \mathrm{~mL} / \mathrm{min}$ 
Tabela 16 - Resultados da análise da associação das apresentações clínicas com os ECM hospitalares pela regressão logística univariada (desenvolvimento do escore DESIRE hospitalar)

\begin{tabular}{|c|c|c|c|c|c|c|}
\hline \multirow{2}{*}{ Variável Resultados } & \multirow{2}{*}{$\begin{array}{c}2863 P \\
N(\%)\end{array}$} & \multicolumn{2}{|c|}{$110(3,8) \mathrm{ECM}$} & \multirow{2}{*}{ OR } & \multirow{2}{*}{$\begin{array}{c}\text { IC } \\
(95 \%)\end{array}$} & \multirow{2}{*}{$\begin{array}{c}\text { Valor } \\
\text { p }\end{array}$} \\
\hline & & $\mathbf{N}$ & $\%$ & & & \\
\hline
\end{tabular}

Apresentação clínica

\begin{tabular}{|c|c|c|c|c|c|c|}
\hline Isquemia silenciosa & $1184(41,4)$ & 34 & 2,9 & 1 & & 0,02 \\
\hline Angina estável & $469(16,4)$ & 14 & 3 & 1,05 & $0,54-1,94$ & \\
\hline Angina instável & $725(25,3)$ & 36 & 5 & 1,77 & $1,09-2,86$ & \\
\hline IM recente & $485(16,9)$ & 26 & 5,4 & 1,92 & $1,13-3,23$ & \\
\hline Até 72 horas & $170(5,9)$ & 6 & 3,5 & 1,02 & $0,39-2,19$ & 0,05 \\
\hline Mais de 72 horas & $315(11)$ & 20 & 6,3 & 1,86 & $1,1-3,02$ & \\
\hline \multicolumn{7}{|c|}{$\begin{array}{l}\text { Síndrome coronária aguda } \\
\text { (AI+IM recente) }\end{array}$} \\
\hline Não & $1653(57,7)$ & 48 & 2,9 & 1 & & $<0,01$ \\
\hline Sim & $1210(42,3)$ & 62 & 5,1 & 1,8 & $1,23-2,66$ & \\
\hline
\end{tabular}

$\mathrm{Al}$ = angina instável; $\mathrm{ECM}=$ eventos cardíacos maiores; IC = intervalo de confiança; $\mathrm{ICP}=$ intervenção coronária percutânea; $\mathrm{IM}=$ infarto do miocárdio; $\mathrm{OR}=$ odds ratio, razão de chances; $\mathrm{P}=$ pacientes; $\mathrm{N}=$ número de casos

Tabela 17 - Resultados da análise da associação da extensão da DAC e Função ventricular esquerda com os ECM hospitalares pela regressão logística univariada (desenvolvimento do escore DESIRE hospitalar)

\begin{tabular}{|c|c|c|c|c|c|c|}
\hline \multirow{2}{*}{ Variável } & $2863 P$ & 110 & ECM & \multirow[t]{2}{*}{ OR } & \multirow{2}{*}{$\begin{array}{c}\text { IC } \\
(95 \%)\end{array}$} & \multirow{2}{*}{$\begin{array}{c}\text { Valor } \\
\mathbf{p}\end{array}$} \\
\hline & $\mathbf{N}(\%)$ & $\mathbf{N}$ & $\%$ & & & \\
\hline
\end{tabular}

Extensão da DAC

\begin{tabular}{lllllll}
\hline Uniarterial & $930(32,5)$ & 18 & 1,9 & 1 & & $<0,01$ \\
Multiarterial & $1933(67,5)$ & 92 & 4,8 & 2,51 & $1,54-4,33$ & \\
\hline
\end{tabular}

\section{Fração de ejeção}

$\begin{array}{llllcll}\mathrm{FE} \geq 40 \% & 2515(91,3) & 92 & 3,7 & 1 & 0,23 \\ \mathrm{FE}<40 \% & 239(8,7) & 13 & 5,4 & 1,53 & 0,8-2,69\end{array}$

DAC = doença arterial coronária; $\mathrm{ECM}=$ eventos cardíacos maiores; IC = intervalo de confiança; $\mathrm{FE}$ = fração de ejeção; $\mathrm{N}$ = número de casos; $\mathrm{OR}$ = odds ratio, razão de chances; $\mathrm{P}$ = pacientes 
Tabela 18 - Resultados da análise da associação das variáveis angiográficas (vasos-alvo) com os ECM hospitalares pela regressão logística univariada (desenvolvimento do escore DESIRE hospitalar)

\begin{tabular}{|c|c|c|c|c|c|c|}
\hline \multirow{2}{*}{ Variável Resultados } & \multirow{2}{*}{$\begin{array}{c}2863 P \\
N(\%)\end{array}$} & \multicolumn{3}{|c|}{$\begin{array}{c}110(3,8 \%) \text { ECM } \\
\text { OR }\end{array}$} & \multirow{2}{*}{$\begin{array}{c}\text { IC } \\
(95 \%)\end{array}$} & \multirow{2}{*}{$\begin{array}{c}\text { Valor } \\
p\end{array}$} \\
\hline & & $\mathbf{N}$ & $\%$ & & & \\
\hline \multicolumn{7}{|l|}{ Vaso-alvo (com PS inclusas) } \\
\hline DA & $886(30,9)$ & 27 & 3 & 1 & & 0,49 \\
\hline$c x$ & $490(17,1)$ & 20 & 4,1 & 1,36 & $0,74-2,44$ & \\
\hline$C D$ & $472(16,5)$ & 15 & 3,2 & 1,05 & $0,54-1,97$ & \\
\hline $\mathrm{DA}+\mathrm{CX}$ & $315(11)$ & 12 & 3,8 & 1,27 & $0,61-2,49$ & \\
\hline $\mathrm{DA}+\mathrm{CD}$ & $269(9,4)$ & 14 & 5,2 & 1,76 & $0,88-3,36$ & \\
\hline$C X+C D$ & $243(8,5)$ & 10 & 4,1 & 1,38 & $0,62-2,81$ & \\
\hline$D A+C X+C D$ & $122(4,3)$ & 8 & 6,6 & 2,26 & $0,93-4,91$ & \\
\hline TCE & $26(0,9)$ & 1 & 3,8 & 1,44 & $0,06-7,21$ & \\
\hline TCE + outros & $40(1,4)$ & 3 & 7,5 & 2,69 & $0,6-8,13$ & \\
\hline
\end{tabular}

\section{Ponte de safena}

\begin{tabular}{lcccccc}
\hline Não & $201(7)$ & 23 & 11,4 & 3,84 & $2,31-6,14$ & \\
\hline Sim & $2662(93)$ & 87 & 3,3 & 1 & & $<0,01$ \\
\hline $\mathbf{N}^{\circ}$ vasos tratados & & & & & & 0,23 \\
\hline Um vaso & $1848(64,5)$ & 62 & 3,4 & 1 & & \\
Dois vasos & $827(28,9)$ & 36 & 4,4 & 1,31 & $0,86-1,99$ & \\
Três vasos & $122(4,3)$ & 8 & 6,6 & 2,05 & $0,88-4,17$ & \\
TCE & $26(0,9)$ & 1 & 3,8 & 1,31 & $0,05-6,3$ & \\
TCE + outro(s) & $40(1,4)$ & 3 & 7,5 & 2,44 & $0,56-7,03$ & \\
\hline
\end{tabular}

Considerando a presença de pelo menos uma lesão com a característica de risco; $\mathrm{CD}=$ artéria coronária direita; $C X=$ artéria circunflexa; $D A=$ artéria descendente anterior; $I C=$ intervalo de confiança; $\mathrm{L}=$ lesões; $\mathrm{N}=$ número de casos; $\mathrm{OR}=$ odds ratio, razão de chances; $\mathrm{P}=$ pacientes; $\mathrm{PS}=$ ponte de safena; TCE = tronco da coronária esquerda 
Tabela 19 - Resultados da análise da associação das variáveis angiográficas (lesões-alvo) com os ECM hospitalares pela regressão logística univariada (desenvolvimento do escore DESIRE hospitalar)

\begin{tabular}{lccccccc}
\hline & Resultados & $2863 \mathrm{P}$ & $110(3,8)$ & $\mathrm{ECM}$ & & IC \\
Variável & $\mathrm{N}(\%)$ & $\mathrm{N}$ & $\%$ & & $(95 \%)$ & $\begin{array}{c}\text { Valor } \\
\mathrm{p}\end{array}$ \\
\cline { 2 - 4 }
\end{tabular}

Lesão de novo

$\begin{array}{ccccccc}\text { Não } & 84(2,9) & 2 & 2,4 & 1 & 0,68 \\ \text { Sim } & 2779(97,1) & 108 & 3,9 & 1,54 & 0,48-10,16\end{array}$

Lesão ostial

$\begin{array}{lccccc}\text { Não } & 2639(92,2) & 99 & 3,8 & 1 & \\ \text { Sim } & 224(7,8) & 11 & 4,9 & 1,34 & 0,67-2,44\end{array}$

Bifurcação§

$\begin{array}{lcccccc}\text { Não } & 2649(92,5) & 100 & 3,8 & 1 & & 0,64 \\ \operatorname{Sim} & 214(7,5) & 10 & 4,7 & 1,27 & 0,61-2,35 & \end{array}$

Oclusão crônica

$\begin{array}{lccccc}\text { Não } & 2823(98,6) & 109 & 3,9 & 1 & \\ \text { Sim } & 40(1,4) & 1 & 2,5 & 0,73 & 0,03-3,36\end{array}$

Trombo

$\begin{array}{ccccccc}\text { Não } & 2721(95) & 95 & 3,5 & 1 & & <0,01 \\ \text { Sim } & 142(5) & 15 & 10,6 & 3,29 & 1,78-5,68\end{array}$

Calcificação

$\begin{array}{lcccccc}\text { Ausente/discreta } & 1934(67,6) & 65 & 3,4 & 1 & & 0,07 \\ \text { Moderada/intensa } & 929(32,4) & 45 & 4,8 & 1,47 & 0,99-2,16 & \end{array}$

Classificação de lesão ${ }^{\sigma}$

$\begin{array}{llllcll}\text { Tipo A ou B } & 1111(38,8) & 16 & 14 & 1 & & <0,01 \\ \text { Tipo C } & 1751(61,2) & 94 & 5,4 & 3,85 & 2,32-6,83 & \end{array}$

Considerando a presença de pelo menos uma lesão com a característica de risco. $\left({ }^{\S}\right)$ Definida pela presença de estenose $\geq 50 \%$ no vaso principal e no vaso lateral, sendo este de diâmetro $\geq 2,0 \mathrm{~mm}$ (estimativa visual); $\left({ }^{\sigma}\right)$ De acordo com a classificação do American College of Cardiology/American Heart Association ${ }^{(81)} ; \mathrm{ECM}=$ eventos cardíacos maiores; IC = intervalo de confiança; OR = odds ratio, razão de chances; $\mathrm{P}=$ pacientes; $\mathrm{N}$ = número de casos 
Tabela 20 - Resultados da análise da associação das variáveis angiográficas com os ECM hospitalares pela regressão logística univariada (desenvolvimento do escore DESIRE hospitalar)

\begin{tabular}{|ccc|cccc} 
Resultados & $2863 \mathrm{P}$ & $110(3,8) \mathrm{ECM}$ & & OR & IC (95\%) & $\begin{array}{c}\text { Valor } \\
\text { Variável }\end{array}$ \\
\cline { 2 - 3 } & $\mathrm{N}(\%)$ & $\mathrm{N}$ & $\%$
\end{tabular}

Lesão longa

$\begin{array}{lllllll}<20 \mathrm{~mm} & 1431(50) & 33 & 2,3 & 1 & <0,01 \\ \geq 20 \mathrm{~mm} & 1432(50) & 77 & 5,4 & 2,4 & 1,6-3,68\end{array}$

Vaso de pequeno calibre ${ }^{\S}$

$\begin{array}{llllll}>2,5 \mathrm{~mm} & 1303(45,5) & 46 & 3,5 & 1 & 0,49\end{array}$

$\begin{array}{llllll}\leq 2,5 \mathrm{~mm} & 1560(54,5) & 64 & 4,1 & 1,17 & 0,8-1,73\end{array}$

Considerando a presença de pelo menos uma lesão com a característica de risco. $\mathrm{ECM}=$ eventos cardíacos maiores; $\mathrm{IC}=$ intervalo de confiança; $\mathrm{N}=$ número de casos $\mathrm{OR}=$ odds ratio, razão de chances; $\mathrm{P}=$ pacientes; $\left({ }^{\#}\right)$ definida como $\geq 20 \mathrm{~mm} ;\left(^{\S}\right)$ definido como diâmetro de referência $\leq 2,5 \mathrm{~mm}$

Uma vez testadas pela regressão logística univariada, as variáveis que apresentaram valor de $\mathrm{p} \leq 0,05$ foram incluídas na análise múltipla. Identificamos colinearidade das seguintes variáveis: insuficiência renal (pelo $\mathrm{ClCr}$ ) com a idade; e da lesão tipo C com: ponte de safena, trombo, calcificação e lesão longa. Desta forma, as variáveis insuficiência renal (pelo $\mathrm{ClCr}$ ) e lesão tipo $\mathrm{C}$ foram excluídas da análise.

As variáveis clínicas e angiográficas selecionadas para esse modelo foram: idade, CRM prévia, doença vascular periférica, insuficiência renal (pela $\mathrm{Cr}$ ), síndrome coronária aguda, doença multiarterial, tratamento de ponte de safena, de lesão com trombo e de lesão longa. 
Na tabela 21 estão relacionadas as variáveis clínicas e angiográficas, para o modelo de regressão logística múltipla.

\begin{tabular}{|c|c|c|c|c|c|c|}
\hline Coeficiente & Estimativa & $\begin{array}{l}\text { Erro } \\
\text { padrão }\end{array}$ & OR & $\begin{array}{c}\text { OR } \\
\text { inferior }\end{array}$ & $\begin{array}{c}\text { OR } \\
\text { superior }\end{array}$ & $\begin{array}{c}\text { valor } \\
p\end{array}$ \\
\hline (Intercepto) & $-7,014$ & 0,7 & 0,001 & 0 & 0,003 & 0 \\
\hline \multicolumn{7}{|l|}{ Variáveis clínicas } \\
\hline Idade & 0,039 & 0,01 & 1,04 & 1,02 & 1,06 & 0 \\
\hline CRM prévia & 0,223 & 0,271 & 1,25 & 0,717 & 2,083 & 0,41 \\
\hline $\begin{array}{l}\text { Doença vascular } \\
\text { periférica }\end{array}$ & 0,648 & 0,405 & 1,912 & 0,805 & 4,005 & 0,109 \\
\hline Insuficiência renal $(\mathrm{Cr})^{\star *}$ & 0,502 & 0,295 & 1,652 & 0,897 & 2,869 & 0,089 \\
\hline $\begin{array}{l}\text { Síndrome coronária } \\
\text { aguda }\end{array}$ & 0,456 & 0,202 & 1,577 & 1,062 & 2,352 & 0,024 \\
\hline
\end{tabular}

\section{Variáveis}

angiográficas

\begin{tabular}{|c|c|c|c|c|c|c|}
\hline Doença multiarterial & 0,303 & 0,21 & 1,354 & 0,9 & 2,052 & 0,148 \\
\hline Ponte de safena & 1,044 & 0,27 & 2,839 & 1,642 & 4,754 & 0 \\
\hline Lesão com trombo & 0,901 & 0,319 & 2,462 & 1,276 & 4,488 & 0,005 \\
\hline Lesão longa ( $\geq 20 \mathrm{~mm})$ & 0,808 & 0,223 & 2,243 & 1,46 & 3,515 & 0 \\
\hline
\end{tabular}

Após a primeira fase, pelo escalonamento (análise stepwise backward pelo critério de Akaike), mantiveram-se as nove variáveis, que agora compõem o modelo final da análise multivariada para ECM hospitalares, sendo cinco delas clínicas e quatro angiográficas. (Tabela 22) A pontuação 
de cada variável foi decidida de acordo com as respectivas razões de chance (OR), variando de 0 a 37 pontos.

Tabela 22 - Modelo final do escore DESIRE hospitalar

\begin{tabular}{|c|c|c|c|c|c|c|c|}
\hline Variáveis & Estimativa & Erro padrão & OR & $\begin{array}{l}\text { OR } \\
\text { inf }\end{array}$ & $\begin{array}{l}\text { OR } \\
\text { sup }\end{array}$ & $\begin{array}{c}\text { Valor } \\
\text { p }\end{array}$ & Pontos" \\
\hline \multicolumn{8}{|l|}{ Clínicas } \\
\hline \multicolumn{8}{|l|}{ Idade (anos) } \\
\hline$\leq 49$ & & & & & & & 0 \\
\hline $50-59$ & & & & & & & 1 \\
\hline $60-69$ & 0,039 & 0,01 & 1,04 & 1,02 & 1,06 & 0 & 2 \\
\hline $70-79$ & & & & & & & 4 \\
\hline$\geq 80$ & & & & & & & 6 \\
\hline CRM prévia & 0,223 & 0,271 & 1,25 & 0,717 & 2,083 & 0,41 & 1 \\
\hline $\begin{array}{l}\text { Doença vascular } \\
\text { periférica }\end{array}$ & 0,648 & 0,405 & 1,912 & 0,805 & 4,005 & 0,109 & 5 \\
\hline $\begin{array}{l}\text { Insuficiência } \\
\text { renal }(\mathrm{Cr})^{\star *}\end{array}$ & 0,502 & 0,295 & 1,652 & 0,897 & 2,869 & 0,089 & 3 \\
\hline $\begin{array}{l}\text { Síndrome } \\
\text { coronária aguda }\end{array}$ & 0,456 & 0,202 & 1,577 & 1,062 & 2,352 & 0,024 & 3 \\
\hline \multicolumn{8}{|l|}{ Angiográficas } \\
\hline $\begin{array}{l}\text { Doença } \\
\text { multiarterial }\end{array}$ & 0,303 & 0,21 & 1,354 & 0,9 & 2,052 & 0,148 & 3 \\
\hline Ponte de safena & 1,044 & 0,27 & 2,839 & 1,642 & 4,754 & 0 & 6 \\
\hline $\begin{array}{l}\text { Lesão com } \\
\text { trombo }\end{array}$ & 0,901 & 0,319 & 2,462 & 1,276 & 4,488 & 0,005 & 5 \\
\hline Lesão longa & 0,808 & 0,223 & 2,243 & 1,46 & 3,515 & 0 & 5 \\
\hline
\end{tabular}


A relação entre os modelos logístico e simplificado apresentou coeficiente $\mathrm{R}^{2}$ igual a 95,1\%, pelo modelo cúbico de regressão. (Figura 9)

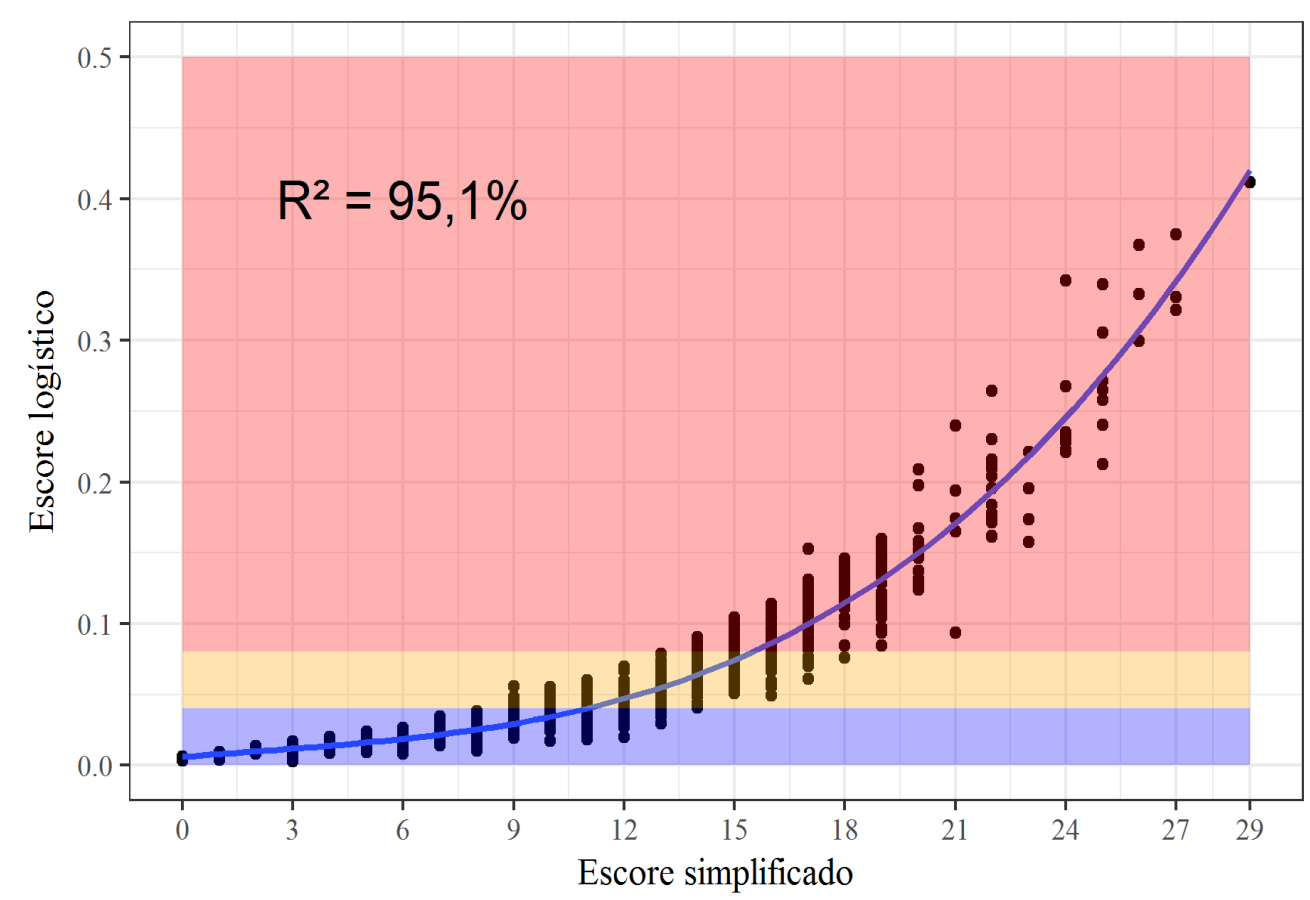

Figura 9 - Relação entre os modelos logístico e simplificado do escore DESIRE hospitalar

A acurácia do modelo nos próprios dados, medida pela área sob a curva ROC, foi igual a 0,74 e 0,73 para os escores logístico e simplificado, respectivamente. (Figura 10) A calibração do modelo, estimado pelo teste de Hosmer-Lemeshow foi igual a 5,8 df8 $(p=0,66)$. 


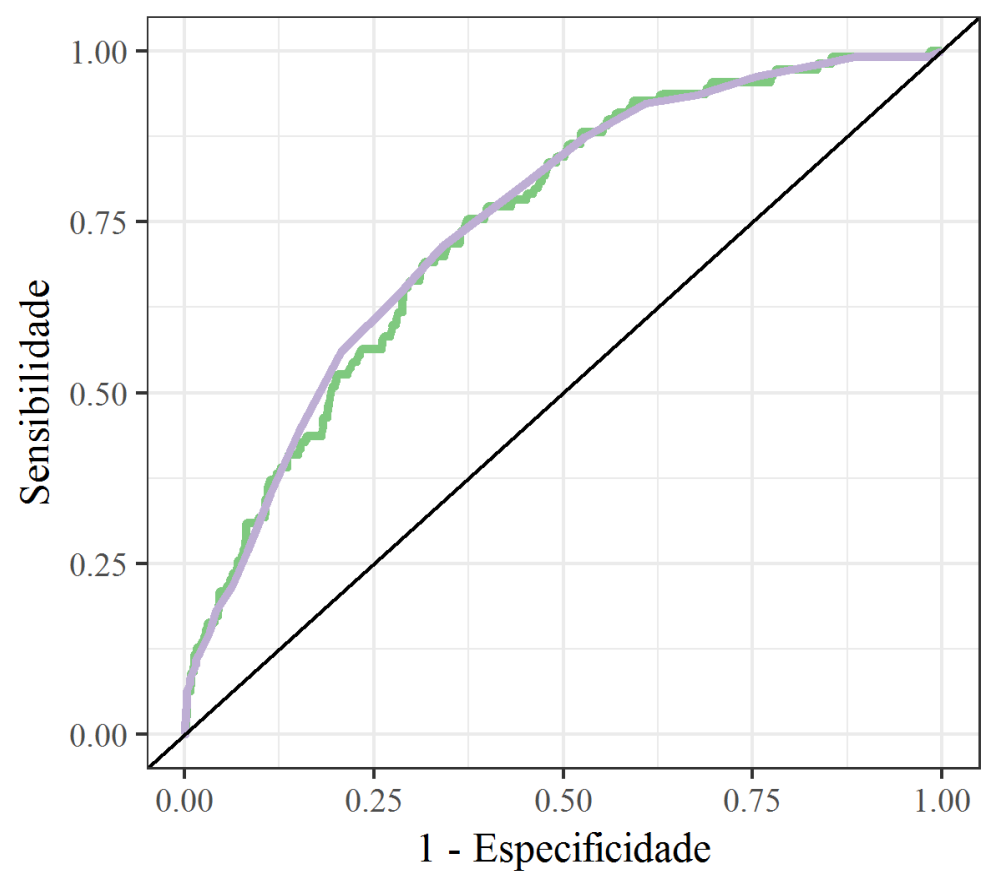

- logístico: AUC $=0.746$ (IC(95\%) $0.789-0.703)$

- Simples: AUC $=0.737$ (IC(95\%) 0.781 - 0.693)

Figura 10 - Acurácia (AUC) dos modelos logístico e simplificado do escore DESIRE hospitalar

Na figura 11, estão descritas as taxas de ECM estimadas de acordo com cada pontuação do escore DESIRE hospitalar. 


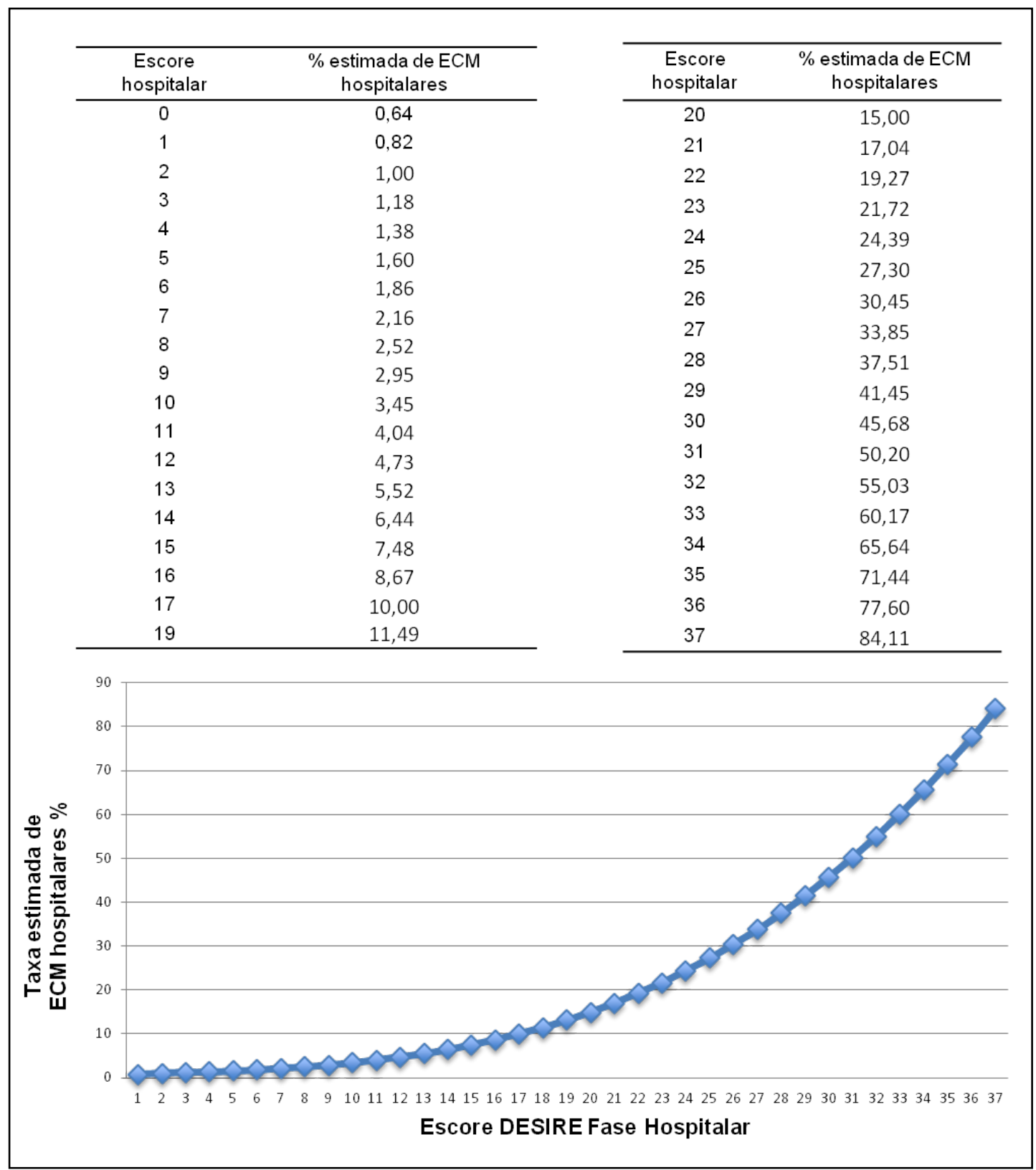

Figura 11- Probabilidades estimadas pelo modelo para cada valor do escore DESIRE hospitalar simplificado. \% = porcentagem; ECM = eventos cardíacos maiores

Desta forma a população B1 foi dividida em três faixas de risco, de acordo com as probabilidades de ECM para cada uma delas. Tendo em conta a taxa de ECM hospitalares na coorte B1 igual a $3,8 \%$ 
(pela classificação do IM pela CKMB $\geq 5$ vezes acima o valor do percentil 99) (tabela 10), foram considerados: de baixo risco os pacientes que tiveram taxa de ECM $\geq 4 \%$ e pontuação até 11 (tabelas 23 e 24); pertencentes ao risco intermediário, os casos com probabilidade entre $>4 \%$ e $\leq 8 \%$ de ECM e pontuação entre 12 e 15, e de alto risco aqueles com probabilidade $>8 \%$ de ECM e com escore DESIRE hospitalar entre 16 e 37 pontos.

Tabela 23 - Faixas de risco para ECM categorizadas pelo escore DESIRE hospitalar (probabilidade de risco).

\begin{tabular}{|c|c|c|c|c|c|c|}
\hline \multirow[b]{3}{*}{$\begin{array}{l}\text { Probabilidade } \\
\text { logística }\end{array}$} & \multicolumn{6}{|c|}{ ECM hospitalar } \\
\hline & \multicolumn{2}{|c|}{ Não } & \multicolumn{2}{|c|}{ Sim } & \multicolumn{2}{|c|}{ Total } \\
\hline & $\mathrm{N}$ & $\%$ & $\mathrm{~N}$ & $\%$ & $N$ & $\%$ \\
\hline$\leq 4 \%$ & 1965 & 97,9 & 42 & 2,1 & 2007 & 100 \\
\hline$>4 \%-\leq 8 \%$ & 558 & 94,3 & 34 & 5,7 & 592 & 100 \\
\hline$>8 \%$ & 226 & 86,9 & 34 & 13,1 & 260 & 100 \\
\hline TOTAL & 2749 & 96,2 & 110 & 3,8 & $2859^{*}$ & 100 \\
\hline
\end{tabular}

$\mathrm{ECM}=$ eventos cardíacos maiores; $\mathrm{N}=$ número de casos.( $\left.{ }^{*}\right)$ Excluídos os pacientes sem informação sobre insuficiência renal.

Tabela 24 - Faixas de risco para ECM categorizadas pelo escore DESIRE hospitalar (pontuação)

\begin{tabular}{|c|c|c|c|c|c|c|}
\hline \multirow{3}{*}{ Pontuação } & \multicolumn{6}{|c|}{ ECM hospitalar } \\
\hline & \multicolumn{2}{|c|}{ Não } & \multicolumn{2}{|c|}{ Sim } & \multicolumn{2}{|c|}{ Total } \\
\hline & $\mathrm{N}$ & $\%$ & $\mathrm{~N}$ & $\%$ & $\mathrm{~N}$ & $\%$ \\
\hline 0 a 11 & 2017 & 97,9 & 43 & 2,1 & 2060 & 100 \\
\hline 12 a 15 & 512 & 92,8 & 40 & 7,2 & 552 & 100 \\
\hline 16 a 37 & 220 & 89,1 & 27 & 10,9 & 247 & 100 \\
\hline TOTAL & 2749 & 96,2 & 110 & 3,8 & $2859^{*}$ & 100 \\
\hline
\end{tabular}

$\mathrm{ECM}=$ eventos cardíacos maiores; $\mathrm{N}=$ número de casos. $\left({ }^{*}\right)$ Excluídos os pacientes sem informação sobre insuficiência renal. 
Associando-se a probabilidade logística e a pontuação obtida, encontramos o disposto na tabela 25.

Tabela 25 - Distribuição dos pacientes da coorte B1 de acordo com as três faixas de risco (probabilidade logística) e pontuação obtida pelo escore DESIRE hospitalar

\begin{tabular}{|c|c|c|c|c|c|c|c|c|}
\hline \multirow{3}{*}{$\begin{array}{l}\text { Probabilidade } \\
\text { logística }\end{array}$} & \multicolumn{8}{|c|}{ Escore simplificado (pontuação) } \\
\hline & \multicolumn{2}{|c|}{0 a 11} & \multicolumn{2}{|c|}{12 a 15} & \multicolumn{2}{|c|}{16 a 37} & \multicolumn{2}{|c|}{ Total } \\
\hline & $\mathrm{N}$ & $\%$ & $\mathrm{~N}$ & $\%$ & $\mathrm{~N}$ & $\%$ & $\mathrm{~N}$ & $\%$ \\
\hline$\leq 4 \%$ & 1923 & 67,3 & 84 & 2,9 & 0 & 0,0 & 2007 & 70,2 \\
\hline$>4 \%-\leq 8 \%$ & 137 & 4,8 & 429 & 15,0 & 26 & 0,9 & 592 & 20,7 \\
\hline$>8 \%$ & 0 & 0,0 & 39 & 1,4 & 221 & 7,7 & 260 & 9,1 \\
\hline TOTAL & 2060 & 72,1 & 552 & 19,3 & 247 & 8,6 & $2859^{*}$ & 100 \\
\hline
\end{tabular}

Acurácia nas faixas de risco: $90 \% ; \mathrm{N}=$ número de casos. ${ }^{*}$ Excluídos os pacientes sem informação sobre insuficiência renal.

$\mathrm{Na}$ figura 12, encontra-se esquematizada a relação das variáveis clínicas e angiográficas, com sua respectiva pontuação, para o cálculo da probabilidade de ECM na fase hospitalar. 


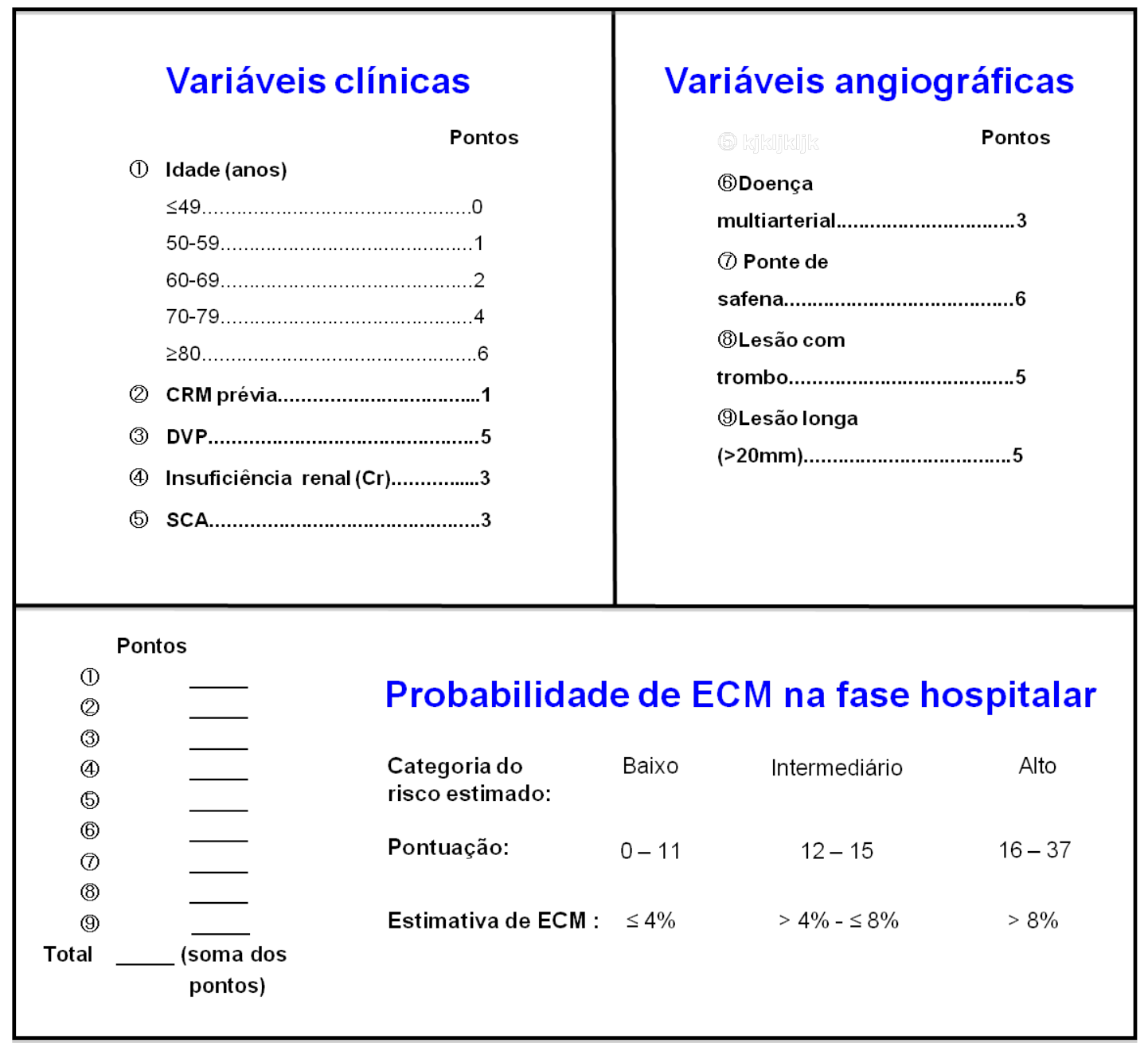

Figura 12 - Esquema do escore DESIRE para cálculo da probabilidade dos ECM hospitalares. $\mathrm{Cr}=$ creatinina; $\mathrm{CRM}=$ cirurgia de revascularização miocárdica; DVP = doença vascular periférica; $\mathrm{SCA}=$ síndrome coronária aguda

\subsubsection{Validação do escore DESIRE Hospitalar}

Uma vez obtido, o escore DESIRE hospitalar foi aplicado nos pacientes das coortes B1 e B2. A partir dos dados da evolução hospitalar, as taxas de ECM estimadas foram comparadas àquelas verificadas, até a alta do hospital. 
Observou-se o aumento estatisticamente significativo nas taxas de ECM a medida em que se aumentou o risco estimado pelo escore DESIRE hospitalar. (Figura 13)

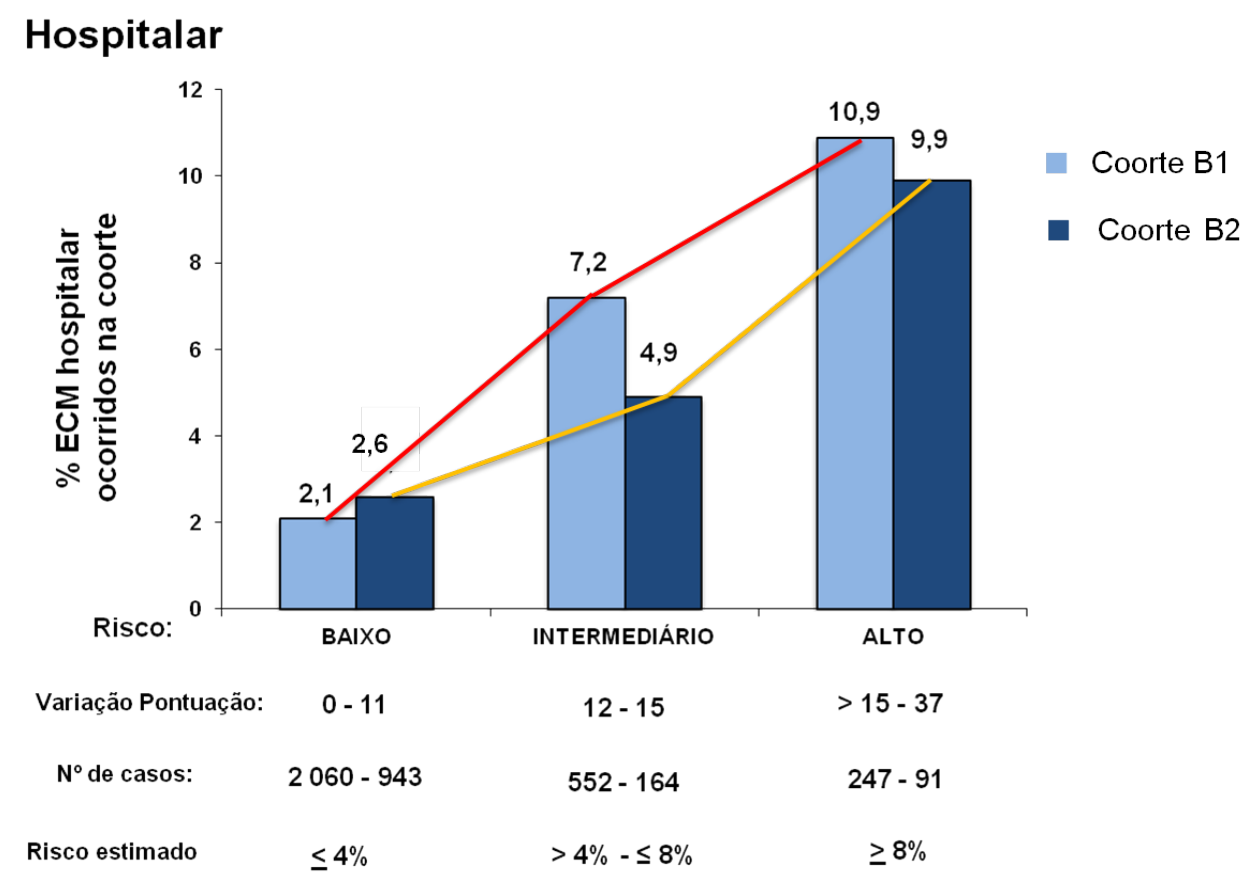

Figura 13 - Validação do escore DESIRE hospitalar. Correlação entre o risco de ECM estimado (faixas de risco e as probabilidades logísticas atribuídas) nos pacientes da coorte B1 e B2 e os respectivos eventos hospitalares ocorridos. ECM = eventos cardíacos maiores

A acurácia do modelo na coorte B2, medida pela área sob a curva ROC, foi igual a 0,64. (Figura 14) A calibração do modelo, estimado pelo teste de Hosmer-Lemeshow foi igual a 26, $3_{\text {df8 }}(p=0,001)$ 


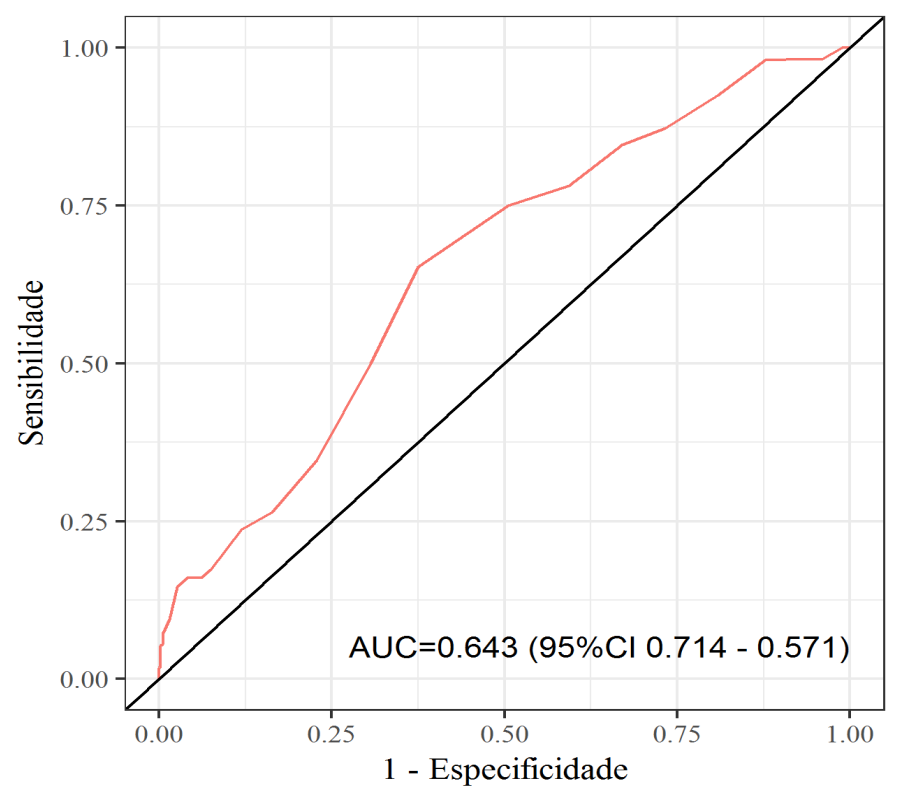

Figura 14 - Acurácia (AUC) dos modelos logístico e simplificado do escore DESIRE hospitalar na coorte B2 (validação); $\mathrm{Cl}=\mathrm{IC}$ = intervalo de confiança

\subsection{Desenvolvimento e validação do escore DESIRE tardio}

\subsubsection{Desenvolvimento do escore DESIRE tardio}

Para a elaboração do escore DESIRE na estimativa dos eventos tardios, testamos a associação das variáveis clínicas e angiográficas, com a ocorrência de ECM combinados na fase evolutiva, pelo modelo de riscos proporcionais de Cox. Nesta etapa, excluímos os 10 casos de óbito ocorridos na fase hospitalar. Os resultados desta análise, nos 2.853 pacientes elegíveis para o seguimento pós-alta do hospital, estão relacionados nas tabelas, 26 a 32 . 
Tabela 26 - Resultados da análise da associação da idade, do gênero e dos fatores de risco com os ECM evolutivos pela regressão de Cox (desenvolvimento do escore DESIRE tardio)

\begin{tabular}{|c|c|c|c|c|c|c|}
\hline \multirow[t]{2}{*}{ Resultado } & \multirow{2}{*}{$\begin{array}{c}2853 P \\
N(\%)\end{array}$} & \multicolumn{2}{|c|}{$173(6,1) \mathrm{ECM}$} & \multirow[b]{2}{*}{ HR } & \multirow[b]{2}{*}{$\begin{array}{c}\text { IC } \\
(95 \%)\end{array}$} & \multirow{2}{*}{$\begin{array}{c}\text { Valor } \\
p\end{array}$} \\
\hline & & $\mathbf{N}$ & $\%$ & & & \\
\hline \multicolumn{7}{|l|}{ Idade } \\
\hline $\begin{aligned} \text { Média } & =65,1 \text { anos } \\
(\mathrm{DP} & =11,0)\end{aligned}$ & - & - & - & 1,00 & $0,99-1,01$ & 0,49 \\
\hline$<70$ anos & $1866(65,4)$ & 115 & 6,2 & 1 & & 0,71 \\
\hline$\geq 70$ anos & $987(34,6)$ & 58 & 5,9 & 0,94 & $0,69-1,29$ & \\
\hline \multicolumn{7}{|l|}{ Sexo } \\
\hline Feminino & $619(21,7)$ & 32 & 5,2 & 1 & & 0,27 \\
\hline Masculino & $2234(78,3)$ & 141 & 6,3 & 1,23 & $0,84-1,81$ & \\
\hline \multicolumn{7}{|l|}{ Fatores de risco } \\
\hline \multicolumn{7}{|l|}{ História familiar de DAC } \\
\hline Não & $1998(70)$ & 123 & 6,2 & 1 & & 0,85 \\
\hline Sim & $855(30)$ & 50 & 5,8 & 0,97 & $0,7-1,35$ & \\
\hline \multicolumn{7}{|l|}{ Diabete melito } \\
\hline Não & $1941(68)$ & 102 & 5,3 & 1 & & 0,01 \\
\hline Sim & $912(32)$ & 71 & 7,8 & 1,51 & $1,12-2,04$ & \\
\hline sem medicação & $49(1,7)$ & 1 & 2 & 0,38 & $0,05-2,75$ & $<0,01$ \\
\hline com medicação oral & $730(25,6)$ & 51 & 7 & 1,36 & $0,97-1,9$ & \\
\hline em uso de insulina & $133(4,7)$ & 19 & 14,3 & 2,78 & $1,7-4,54$ & \\
\hline \multicolumn{7}{|l|}{ Hipertensão arterial } \\
\hline Não & $643(22,5)$ & 30 & 4,7 & 1 & & 0,08 \\
\hline $\operatorname{Sim}$ & $2210(77,5)$ & 143 & 6,5 & 1,4 & $0,94-2,07$ & \\
\hline \multicolumn{7}{|l|}{ Dislipidemia } \\
\hline Não & $1010(35,4)$ & 54 & 5,3 & 1 & & 0,23 \\
\hline Sim & $1843(64,6)$ & 119 & 6,5 & 1,22 & $0,88-1,68$ & \\
\hline \multicolumn{7}{|l|}{ Tabagismo } \\
\hline Nunca & $2031(71,2)$ & 112 & 5,5 & 1 & & 0,04 \\
\hline Ex-tabagista & $576(20,2)$ & 48 & 8,3 & 1,56 & $1,11-2,19$ & \\
\hline Fumante atual & $246(8,6)$ & 13 & 5,3 & 0,96 & $0,54-1,7$ & \\
\hline \multicolumn{7}{|l|}{ Obesidade* } \\
\hline Não & $2001(70,5)$ & 116 & 5,8 & 1 & & 0,32 \\
\hline Sim & $838(29,5)$ & 57 & 6,8 & 1,18 & $0,86-1,61$ & \\
\hline
\end{tabular}

DAC = doença arterial coronária; $\mathrm{ECM}=$ eventos cardíacos maiores; IC = intervalo de confiança; $\mathrm{HR}$ = hazard ratio, taxa de risco; $\mathrm{N}=$ número de casos $\mathrm{P}=$ pacientes;

$\left(^{\dagger}\right)$ Definida pelo IMC $>30 \mathrm{~kg} / \mathrm{m}^{2}$ 
Tabela 27 - Resultados da análise da associação dos eventos e procedimentos cardiovasculares prévios com os ECM evolutivos pela regressão de Cox (desenvolvimento do escore DESIRE tardio)

\begin{tabular}{|c|c|c|c|c|c|c|}
\hline \multirow{2}{*}{$\begin{array}{l}\text { Resultados } \\
\text { Variável } \\
\end{array}$} & \multirow{2}{*}{$\begin{array}{c}2853 \mathrm{P} \\
\mathrm{N}(\%) \\
\end{array}$} & \multicolumn{2}{|c|}{$173(6,1) \mathrm{ECM}$} & \multirow[t]{2}{*}{ HR } & \multirow{2}{*}{$\begin{array}{c}\text { IC } \\
(95 \%)\end{array}$} & \multirow{2}{*}{$\begin{array}{c}\text { Valor } \\
\text { p }\end{array}$} \\
\hline & & $\mathbf{N}$ & $\%$ & & & \\
\hline \multicolumn{7}{|c|}{$\begin{array}{l}\text { Eventos e procedimentos } \\
\text { cardiovasculares prévios }\end{array}$} \\
\hline \multicolumn{7}{|l|}{ IM } \\
\hline Não & $2176(76,3)$ & 121 & 5,6 & 1 & & 0,05 \\
\hline Sim & $677(23,7)$ & 52 & 7,7 & 1,4 & $1,01-1,93$ & \\
\hline \multicolumn{7}{|l|}{ AVE } \\
\hline Não & $2791(97,8)$ & 167 & 6 & 1 & & 0,27 \\
\hline Sim & $62(2,2)$ & 6 & 9,7 & 1,64 & $0,72-3,69$ & \\
\hline \multicolumn{7}{|l|}{ ICP } \\
\hline Não & $2150(75,4)$ & 121 & 1 & 1 & & 0,1 \\
\hline Sim & $703(24,6)$ & 52 & 7,4 & 1,32 & $0,95-1,82$ & \\
\hline \multicolumn{7}{|l|}{ CRM } \\
\hline Não & 2168 (76) & 108 & 5 & 1 & & $<0,01$ \\
\hline Sim & $685(24)$ & 65 & 9,5 & 1,94 & $1,42-2,63$ & \\
\hline
\end{tabular}

\section{Comorbidades}

Doença vascular periférica

$\begin{array}{lrrrrr}\text { Não } & 2770(97,1) & 163 & 5,9 & 1 & \\ \text { Sim } & 83(2,9) & 10 & 12 & 2,11 & 1,11-3,99\end{array}$

Insuficiência renal $(\mathrm{Cr})^{* *}$

$\begin{array}{lrrrrr}\text { Não } & 2650(93) & 154 & 5,8 & 1 & \\ \text { Sim } & 199(7) & 18 & 9 & 1,58 & 0,97-2,57\end{array}$

Insuficiência Renal $(\mathrm{ClCr})^{\star * *}$

$\begin{array}{llllll}\text { Não } & 2119(76,1) & 116 & 5,5 & 1 & 0,05 \\ \text { Sim } & 664(23,9) & 51 & 7,7 & 1,41 & 1,01-1,96\end{array}$

AVE = acidente vascular encefálico; $\mathrm{ClCr}=$ clearance de creatinina; $\mathrm{CR}$ = creatinina; $\mathrm{CRM}=$ cirurgia de revascularização miocárdica; $\mathrm{ECM}=$ eventos cardíacos maiores; $\mathrm{HR}=$ hazard ratio, taxa de risco; IC = intervalo de confiança; ICP = intervenção coronária percutânea; $\mathrm{IM}=$ infarto do miocárdio; $\mathrm{N}=$ número de casos; $\mathrm{P}=$ pacientes; ${ }^{* *}$ Definida pela creatinina $\geq 1,5 \mathrm{mg} / \mathrm{dl}$; ${ }^{* * *}$ Definida pelo clearance de creatinina $<60 \mathrm{~mL} / \mathrm{min}$ 
Tabela 28 - Resultados da análise da associação das apresentações clínicas com os ECM evolutivos pela regressão de Cox (desenvolvimento do escore DESIRE tardio)

\begin{tabular}{|c|c|c|c|c|c|c|}
\hline \multirow{2}{*}{ Variável Resultados } & \multirow{2}{*}{$\begin{array}{r}2853 P \\
N(\%)\end{array}$} & \multicolumn{2}{|c|}{$173(6,1) \mathrm{ECM}$} & \multirow[t]{2}{*}{ HR } & \multirow[t]{2}{*}{$\begin{array}{c}\text { IC } \\
(95 \%)\end{array}$} & \multirow[t]{2}{*}{$\begin{array}{c}\text { Valor } \\
p^{\#}\end{array}$} \\
\hline & & $\mathbf{N}$ & $\%$ & & & \\
\hline \multicolumn{7}{|l|}{ Apresentação clínica } \\
\hline Isquemia silenciosa & $1183(41,5)$ & 55 & 4,6 & 1 & & 0,01 \\
\hline Angina estável & $469(16,4)$ & 25 & 5,3 & 1,14 & $0,71-1,83$ & \\
\hline Angina instável & $723(25,3)$ & 53 & 7,3 & 1,6 & $1,09-2,33$ & \\
\hline IM recente & $478(16,8)$ & 40 & 8,4 & 1,84 & $1,23-2,77$ & \\
\hline Até 72 horas & $167(5,9)$ & 11 & 6,6 & 1,19 & $0,64-2,2$ & 0,05 \\
\hline Mais de 72 horas & $311(10,9)$ & 29 & 9,3 & 1,71 & $1,14-2,55$ & \\
\hline \multicolumn{7}{|l|}{$\begin{array}{l}\text { Síndrome coronária aguda } \\
\text { (AI + IM recente) }\end{array}$} \\
\hline Sim & $1201(42,1)$ & 93 & 7,7 & 1,63 & $1,21-2,19$ & \\
\hline
\end{tabular}

$\mathrm{Al}=$ angina instável; $\mathrm{ECM}=$ eventos cardíacos maiores; $\mathrm{IM}=$ infarto do miocárdio; $\mathrm{IC}=$ intervalo de confiança; $\mathrm{HR}=$ hazard ratio, taxa de risco; $\mathrm{N}=$ número de casos; $\mathrm{P}=$ pacientes;

Tabela 29 - Resultados da análise da associação da extensão da DAC e Função ventricular esquerda com os ECM evolutivos pela regressão de Cox (desenvolvimento do escore DESIRE tardio)

\begin{tabular}{ccccccc}
\hline Resultados & $2853 \mathrm{P}$ & 173 (6,1) ECM & HR & $\begin{array}{c}\text { IC } \\
(95 \%)\end{array}$ & $\begin{array}{c}\text { Valor } \\
\text { Variável }\end{array}$ \\
\cline { 2 - 4 } & $\mathrm{N}(\%)$ & $\mathrm{N}$ & $\%$ & & & \\
\hline
\end{tabular}

\section{Extensão da DAC}

\begin{tabular}{lrrrrrr}
\hline Uniarterial & $930(32,5)$ & 46 & 4,9 & 1 & & $<0,01$ \\
Multiarterial & $1933(67,5)$ & 191 & 9,9 & 12,1 & $1,52-2,96$ & \\
\hline
\end{tabular}

Fração de ejeção, \%

$\begin{array}{lcccccc}\mathrm{FE} \geq 40 \% & 237(8,6) & 25 & 10,5 & 1,98 & 1,29-3,03 & <0,01 \\ \mathrm{FE}<40 \% & 2508(91,4) & 137 & 5,5 & 1 & & \end{array}$

$\overline{\mathrm{DAC}}=$ doença arterial coronária; $\mathrm{ECM}=$ eventos cardíacos maiores; FE = fração de ejeção; $\mathrm{HR}=$ hazard ratio, taxa de risco; $\mathrm{IC}=$ intervalo de confiança; $\mathrm{N}=$ número de casos; $\mathrm{P}=$ paciente 
Tabela 30 - Resultados da análise da associação das variáveis angiográficas (vasos-alvo) com os ECM evolutivos pela regressão de Cox (desenvolvimento do escore DESIRE tardio)

\begin{tabular}{|c|c|c|c|c|}
\hline Variável Resultados & 2853 & $\begin{array}{l}173(6,1 \%) \text { ECM } \\
\text { HR }\end{array}$ & $\begin{array}{c}\text { IC } \\
(95 \%)\end{array}$ & $\begin{array}{l}\text { Valor } \\
p\end{array}$ \\
\hline & $\mathbf{N}(\%)$ & $\%$ & & \\
\hline
\end{tabular}

território-alvo (com PS inclusas)

$\begin{array}{lcccccc}\text { DA } & 880(30,8) & 32 & 3,6 & 1 & & <0,01 \\ C X & 490(17,2) & 33 & 6,7 & 1,87 & 1,15-3,04 \\ C D & 468(16,4) & 24 & 5,1 & 1,43 & 0,84-2,43 \\ D A+C X & 315(11) & 31 & 9,8 & 2,81 & 1,72-4,61 \\ D A+C D & 269(9,4) & 14 & 5,2 & 1,44 & 0,77-2,7 \\ C X+C D & 243(8,5) & 23 & 9,5 & 2,65 & 1,55-4,52 \\ D A+C X+C D & 122(4,3) & 12 & 9,8 & 2,83 & 1,46-5,5 \\ \text { TCE } & 26(0,9) & 1 & 3,8 & 1,03 & 0,14-7,53 \\ \text { TCE + outros } & 40(1,4) & 3 & 7,5 & 2,12 & 0,65-6,93\end{array}$

\section{Ponte de safena}

\begin{tabular}{lcccccc}
\hline Não & $201(7)$ & 65 & 32,3 & 4,47 & $3,24-6,16$ & \\
Sim & $2662(93)$ & 320 & 12,0 & 1 & & $<0,01$ \\
\hline $\begin{array}{l}\mathbf{N}^{0} \text { territórios } \\
\text { tratados }\end{array}$ & $1838(64,4)$ & 89 & 4,8 & 1 & & 0,01 \\
\hline Um & $827(29)$ & 68 & 8,2 & 1,72 & $1,26-2,37$ & \\
Dois & $122(4,3)$ & 12 & 9,8 & 2,12 & $1,16-3,87$ & \\
Três & $26(0,9)$ & 1 & 3,8 & 0,77 & $0,11-5,51$ & \\
TCE & $40(1,4)$ & 3 & 7,5 & 1,58 & $0,5-5$ & \\
TCE + outro(s) & 40,5 & & & & &
\end{tabular}

Considerando a presença de pelo menos uma lesão com a característica de risco; $C D=$ artéria coronária direita; $\mathrm{CX}=$ artéria circunflexa; $\mathrm{DA}=$ artéria descendente anterior; $\mathrm{ECM}=$ eventos cardíacos maiores; $\mathrm{HR}=$ hazard ratio, taxa de risco; $\mathrm{IC}=$ intervalo de confiança; $\mathrm{L}=$ lesões; $\mathrm{N}=$ número de casos; $\mathrm{P}$ = pacientes; $\mathrm{PS}=$ ponte de safena; $\mathrm{TCE}=$ tronco da coronária esquerda 
Tabela 31 - Resultados da análise da associação das variáveis angiográficas (lesões-alvo) com os ECM evolutivos pela regressão de Cox (desenvolvimento do escore DESIRE tardio)

\begin{tabular}{lcccccc}
\hline & $2853 \mathrm{P}$ & $173(6,1) \mathrm{ECM}$ & HR & $\begin{array}{c}\text { IC } \\
(95 \%)\end{array}$ & $\begin{array}{c}\text { Valor } \\
\mathrm{p}\end{array}$ \\
\cline { 2 - 3 } Variável & $\mathrm{N}(\%)$ & $\mathrm{N}$ & $\%$ & & & \\
\hline
\end{tabular}

Lesão de novo
Não
$84(2,9)$
6
$7,1 \quad 1$
$\operatorname{Sim}$
$2769(97,1)$
167
$6,0 \quad 0,85$
$0,38-1,92$

0,71

Lesão ostial*

Não

$2630(92,2) \quad 149 \quad 5,7$

Sim

Bifurcação ${ }^{\S}$

Não

$$
2639(92,5) \quad 155
$$

$5,9 \quad 1$

0,14

Sim

$214(7,5) \quad 18 \quad 8,4 \quad 1,47 \quad 0,9-2,4$

Oclusão crônica

Não

$2813(98,6) \quad 169 \quad 6,0 \quad 1$

Sim

$$
40(1,4)
$$

$4 \quad 10,0$

1,73

$0,64-4,67$

Trombo

Não

Sim

Calcificação

Ausente/discreta

Moderada/intensa

Classificação de lesão ${ }^{\sigma}$

$$
2714(95,1) \quad 159
$$$$
139(4,9)
$$

14

5,9

1

0,06

10,1

1,75

$1,02-3,03$

Tipo A ou B

Tipo C presença de estenose $\geq 50 \%$ no vaso principal e no vaso lateral, sendo este de diâmetro $\geq 2,0 \mathrm{~mm}$ (estimativa visual); $\left({ }^{\sigma}\right)$ De acordo com a classificação do American College of Cardiology/American Heart Association ${ }^{(79)}$; $\mathrm{ECM}=$ eventos cardíacos maiores; IC = intervalo de confiança; $\mathrm{N}=$ número de casos; $\mathrm{HR}=$ hazard ratio, taxa de risco; $\mathrm{P}=$ pacientes 
Tabela 32 - Resultados da análise da associação das variáveis angiográficas com os ECM evolutivos pela regressão de Cox (desenvolvimento do escore DESIRE tardio)

\begin{tabular}{|c|c|c|c|c|c|c|}
\hline \multirow{2}{*}{ Variável } & \multirow{2}{*}{$\begin{array}{c}2853 \mathrm{P} \\
\mathrm{N}(\%)\end{array}$} & \multicolumn{2}{|c|}{$173(6,1) \mathrm{ECM}$} & \multirow{2}{*}{ HR } & \multirow{2}{*}{ IC (95\%) } & \multirow{2}{*}{$\begin{array}{c}\text { Valor } \\
\mathbf{p}\end{array}$} \\
\hline & & $\mathbf{N}$ & $\%$ & & & \\
\hline \multicolumn{7}{|l|}{ Lesão longa ${ }^{\#}$} \\
\hline$<20 \mathrm{~mm}$ & $1425(49,9)$ & 71 & 5,0 & 1 & & 0,01 \\
\hline$\geq 20 \mathrm{~mm}$ & $1428(50,1)$ & 102 & 7,1 & 1,45 & $1,07-1,97$ & \\
\hline \multicolumn{7}{|c|}{ Vaso de pequeno calibre ${ }^{\S}$} \\
\hline$>2,5 \mathrm{~mm}$ & $1298(45,5)$ & 56 & 4,3 & 1 & & $<0,01$ \\
\hline$\leq 2,5 \mathrm{~mm}$ & $1555(54,5)$ & 117 & 7,5 & 1,77 & $1,29-2,44$ & \\
\hline
\end{tabular}

Considerando a presença de pelo menos uma lesão com a característica de risco. ECM = eventos cardíacos maiores; $\mathrm{IC}=$ intervalo de confiança; $\mathrm{N}=$ número de casos; $\mathrm{P}=$ pacientes; $\mathrm{HR}$ = hazard ratio, taxa de risco; $\left({ }^{\#}\right)$ definida como $\geq 20 \mathrm{~mm} ;\left(^{\S}\right)$ definido como diâmetro de referência $\leq 2,5 \mathrm{~mm}$

As variáveis selecionadas para o modelo de regressão de Cox inicial foram: diabete melito $(p<0,001)$, tabagismo $(p=0,04)$, IM prévio $(p=0,05)$ CRM prévia $(p<0,01)$, doença vascular periférica $(p=0,04)$, insuficiência renal $(p=0,05)$, síndrome coronária aguda $(p<0,01)$, doença multiarterial (lesão em dois vasos, em três vasos, no TCE isolado e no TCE associado a outros vasos) $(p<0,01)$, ponte de safena $(p<0,001)$, disfunção ventricular esquerda ( $F E<40 \%)$, lesão ostial $(p<0,01)$, lesão tipo $C(p<0,01)$, lesão longa $(p=0,01)$ e vaso de pequeno calibre $(p<0,01)$. A idade foi inserida por ser considerada relevante neste contexto, a despeito de não ter se 
caracterizado como uma variável com efeito prognóstico na fase tardia. (Tabela 33)

Tabela 33- Variáveis do modelo inicial de regressão de Cox para o escore DESIRE tardio

\begin{tabular}{|c|c|c|c|c|c|c|}
\hline \multirow[b]{2}{*}{ Variáveis clínicas } & \multirow[t]{2}{*}{ Coeficien } & \multirow[t]{2}{*}{$\begin{array}{l}\text { EP } \\
\text { coef }\end{array}$} & \multirow[t]{2}{*}{ HR } & \multirow[t]{2}{*}{$\begin{array}{c}\mathrm{HR} \\
\text { (inferior) }\end{array}$} & \multicolumn{2}{|c|}{$\begin{array}{c}\mathrm{HR} \\
\text { (superior) }\end{array}$} \\
\hline & & & & & & \\
\hline Idade & $-0,007$ & 0,009 & $-0,767$ & 0,993 & 0,976 & 1,011 \\
\hline $\begin{array}{l}\text { Diabete melito } \\
\text { só medicação oral }\end{array}$ & 0,416 & 0,178 & 2,336 & 1,516 & 1,069 & 2,149 \\
\hline em uso de insulina & 1,021 & 0,260 & 3,929 & 2,777 & 1,668 & 4,622 \\
\hline CRM prévia & 0,230 & 0,213 & 1,082 & 1,259 & 0,830 & 1,910 \\
\hline IM prévio & 0,069 & 0,187 & 0,370 & 1,072 & 0,743 & 1,545 \\
\hline ICP prévia & 0,194 & 0,183 & 1,062 & 1,214 & 0,849 & 1,736 \\
\hline Doença vascular periférica & 0,530 & 0,351 & 1,511 & 1,700 & 0,854 & 3,381 \\
\hline Insuficiência renal $(\mathrm{Cr})^{\star *}$ & 0,218 & 0,207 & 1,054 & 1,243 & 0,829 & 1,865 \\
\hline
\end{tabular}

\section{Apresentação clínica}

\begin{tabular}{lllllll}
\hline SCA & 0,342 & 0,164 & 2,083 & 1,408 & 1,02 & 1,942
\end{tabular}

Variáveis angiográficas

Multiarterial

$\begin{array}{llllll}0,354 & 0,173 & 2,052 & 1,425 & 1,016 & 1,999\end{array}$

FE $<40 \%$

$\begin{array}{llllll}0,469 & 0,228 & 2,054 & 1,599 & 1,022 & 2,501\end{array}$

Ponte de safena

$\begin{array}{llllll}0,993 & 0,224 & 4,432 & 2,699 & 1,74 & 4,186\end{array}$

Trombo

$\begin{array}{llllll}0,428 & 0,295 & 1,454 & 1,535 & 0,861 & 2,735\end{array}$

Lesão longa

$\begin{array}{llllll}0,208 & 0,17 & 1,222 & 1,231 & 0,882 & 1,717\end{array}$

Vaso de pequeno calibre

$0,413 \quad 0,178 \quad 2,325 \quad 1,511 \quad 1,067 \quad 2,141$

$\mathrm{CR}$ = creatinina; $\mathrm{CRM}$ = cirurgia de revascularização miocárdica; $\mathrm{EP}$ coef = erro padrão coeficiente; FE = fração de ejeção; $H R=$ hazard ratio, taxa de risco; $I M=$ infarto miocárdio; $I C P=$ intervenção coronária percutânea; $\mathrm{SCA}$ = síndrome coronária aguda; ${ }^{* *}$ Definida pela Creatinina $\geq 1,5 \mathrm{mg} / \mathrm{dl}$;

As oito variáveis que permaneceram no modelo, selecionadas pelo critério de Akaike, estão descritas na tabela 34, com seus respectivos riscos 
de ECM e pontuações correspondentes, compondo assim o modelo final do escore DESIRE tardio. A pontuação total variou de 0 a 45.

Tabela 34 - Modelo final do escore DESIRE tardio

\begin{tabular}{|c|c|c|c|c|c|c|c|}
\hline Variável & Coeficiente & EP coef & $\mathrm{HR}$ & HR inf & HR sup & Valor $p$ & Pontos \\
\hline \multicolumn{8}{|l|}{ Clínicas } \\
\hline \multicolumn{8}{|l|}{ Diabete melito } \\
\hline $\begin{array}{c}\text { Só medicação } \\
\text { oral }\end{array}$ & 0,389 & 0,175 & 1,476 & 1,048 & 2,08 & 0,026 & 4 \\
\hline $\begin{array}{l}\text { em uso de } \\
\text { insulina }\end{array}$ & 0,958 & 0,259 & 2,608 & 1,571 & 4,33 & $<0,001$ & 9 \\
\hline CRM prévia & 0,201 & 0,208 & 1,222 & 0,813 & 1,837 & 0,335 & 2 \\
\hline $\begin{array}{l}\text { Doença vascular } \\
\text { periférica }\end{array}$ & 0,559 & 0,345 & 1,749 & 0,89 & 3,439 & 0,105 & 6 \\
\hline SCA & 0,421 & 0,159 & 1,523 & 1,115 & 2,08 & 0,008 & 5 \\
\hline \multicolumn{8}{|l|}{ Angiográficas } \\
\hline $\begin{array}{l}\text { Doença } \\
\text { multiarterial }\end{array}$ & 0,395 & 0,165 & 1,484 & 1,074 & 2,05 & 0,017 & 4 \\
\hline $\mathrm{FE}<40 \%$ & 0,527 & 0,22 & 1,694 & 1,101 & 2,607 & 0,017 & 6 \\
\hline Ponte de safena & 1,05 & 0,211 & 2,857 & 1,891 & 4,318 & $<0,001$ & 8 \\
\hline $\begin{array}{l}\text { Vaso de pequeno } \\
\text { calibre }\end{array}$ & 0,441 & 0,176 & 1,555 & 1,102 & 2,194 & 0,012 & 5 \\
\hline
\end{tabular}

Por meio do modelo cúbico de regressão, obteve-se um coeficiente $R^{2}$ igual a $95,3 \%$, na relação entre os modelos simplificados e brutos. (Figura 15) 


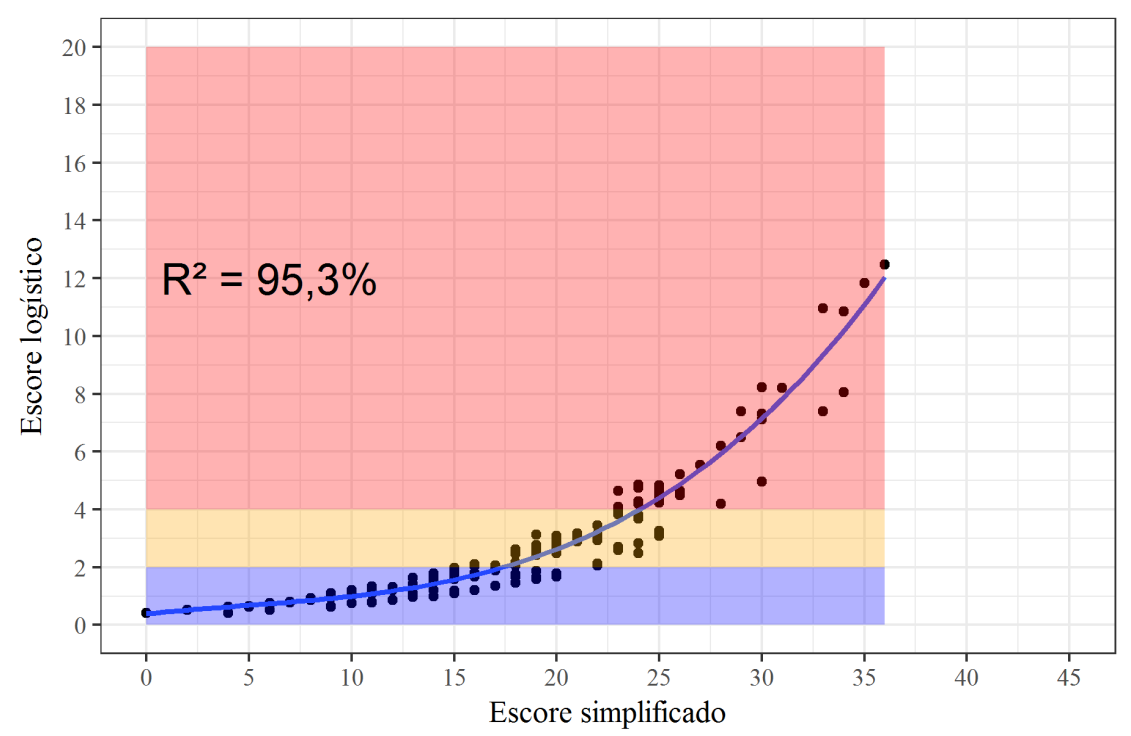

Figura 15 - Relação entre os modelos logístico e simplificado do escore DESIRE tardio

A acurácia do modelo, ao longo do tempo, foi igual a 0,65 (Figura 16) A calibração do modelo, estimado pelo teste de Hosmer-Lemeshow foi igual a $2,4_{\text {df8 }}(p=0,96)$ 


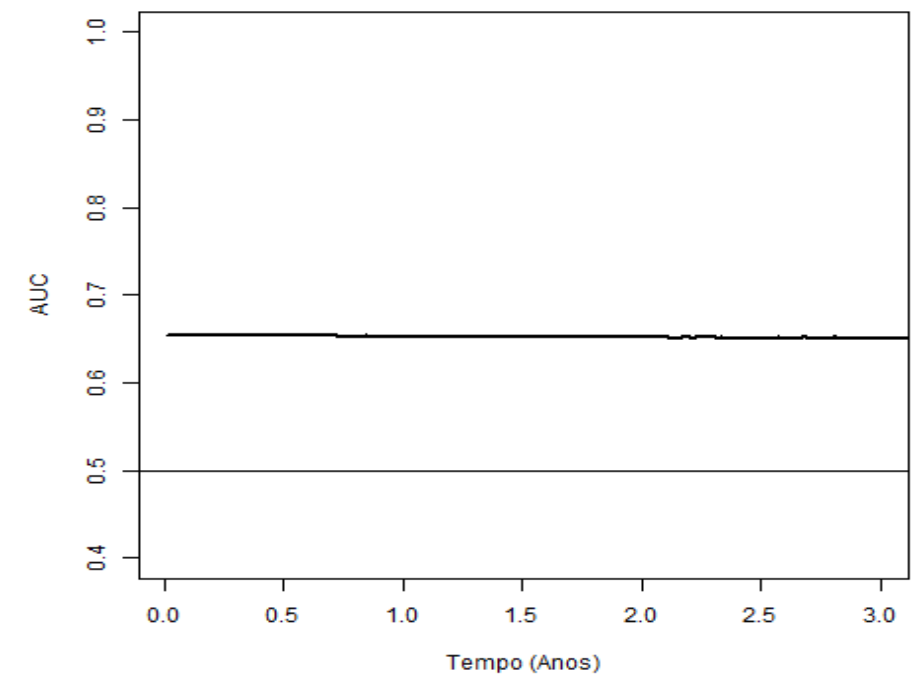

Figura 16 - Acurácia (AUC) do escore DESIRE tardio.

Seguem listadas, na figura 17, as probabilidades de eventos de acordo com o cálculo do escore DESIRE tardio. 


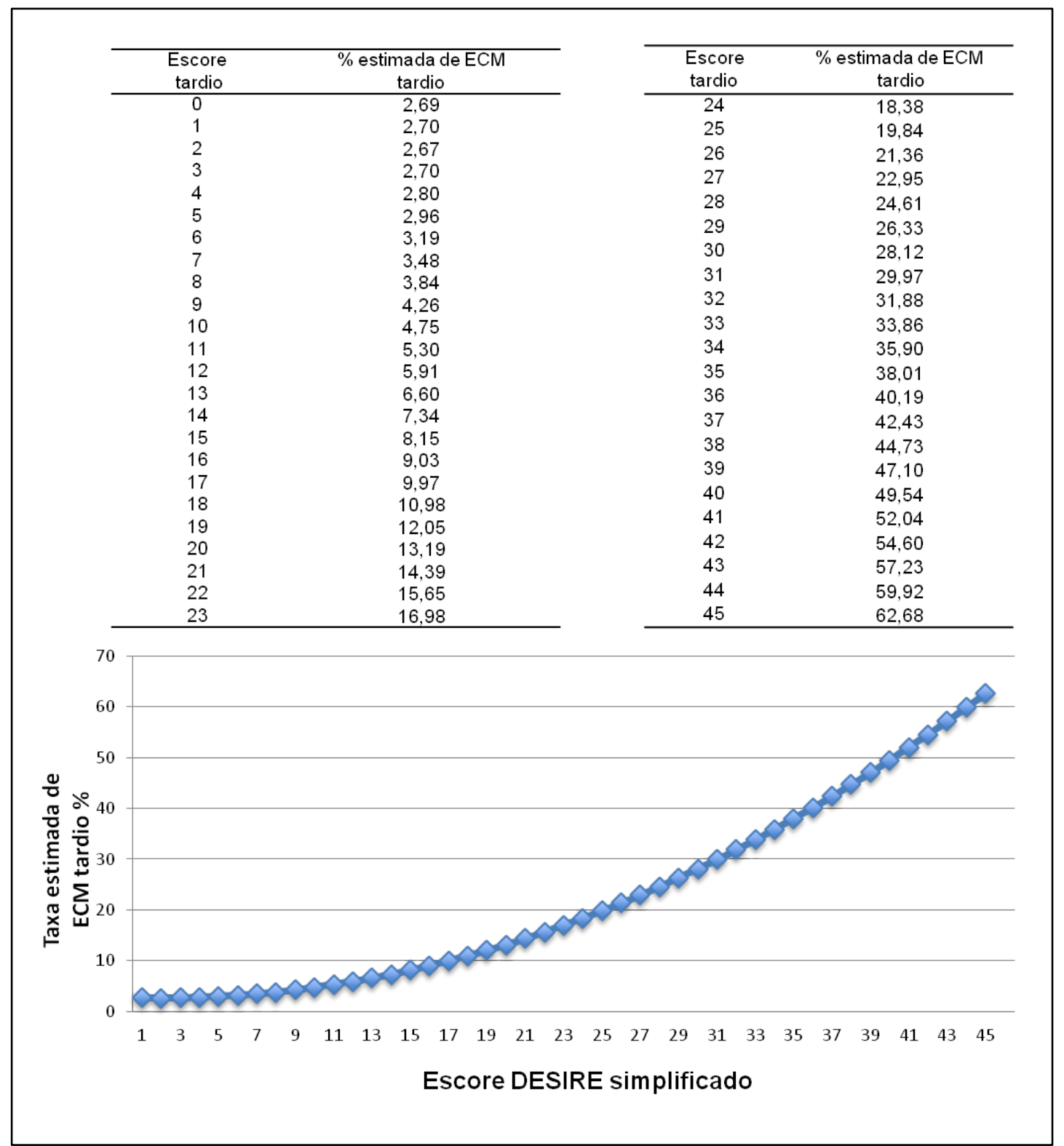

Figura 17- Probabilidades estimadas pelo modelo para cada valor do escore DESIRE tardio simplificado. $\%=$ porcentagem; ECM = eventos cardíacos maiores

Segundo o escore DESIRE tardio, categorizamos três grupos, de acordo com as faixas de risco para ECM na evolução $(6,3 \%)$ (Tabela 10). Foram considerados de baixo risco os pacientes que tivessem taxa de ECM menor ou igual à média ocorrida nessa coorte $(\leq 6 \%)$; risco intermediário ( $>6 \%-\leq 12 \%$ ), os que tiveram o dobro da média e os com 
mais que o dobro da média foram considerados de alto risco ( $>12 \%)$. (Tabelas 35 a 37 )

\begin{tabular}{|c|c|c|c|c|c|c|}
\hline \multirow[b]{3}{*}{$\begin{array}{l}\text { Probabilidade } \\
\text { logística }\end{array}$} & \multicolumn{6}{|c|}{ ECM tardios } \\
\hline & \multicolumn{2}{|c|}{ Não } & \multicolumn{2}{|c|}{ Sim } & \multicolumn{2}{|c|}{ Total } \\
\hline & $\mathbf{N}$ & $\%$ & $\mathbf{N}$ & $\%$ & $\mathbf{N}$ & $\%$ \\
\hline$\leq-6 \%$ & 1795 & 96,3 & 69 & 3,7 & 1864 & 100 \\
\hline$>6 \%-\leq 12 \%$ & 579 & 92,3 & 48 & 7,7 & 627 & 100 \\
\hline$>12 \%$ & 197 & 81,4 & 45 & 18,6 & 242 & 100 \\
\hline Total & 2571 & 94,1 & 162 & 5,9 & $2733^{*}$ & 100 \\
\hline
\end{tabular}

$\mathrm{ECM}=$ eventos cardíacos maiores; $\mathrm{N}=$ número de casos. $\left({ }^{*}\right)$ Excluídos pacientes que tiveram óbito, sem informação sobre insuficiência renal e ou sem seguimento clínico

Tabela 36 - Faixas de risco para ECM categorizadas pelo Escore DESIRE tardio (Pontuação)

\begin{tabular}{|c|c|c|c|c|c|c|}
\hline \multirow{3}{*}{ Pontuação } & \multicolumn{6}{|c|}{ ECM tardios } \\
\hline & \multicolumn{2}{|c|}{ Não } & \multicolumn{2}{|c|}{ Sim } & \multicolumn{2}{|c|}{ Total } \\
\hline & $\mathbf{N}$ & $\%$ & $\mathbf{N}$ & $\%$ & $\mathbf{N}$ & $\%$ \\
\hline 0 a 12 & 1737 & 96,2 & 69 & 3,8 & 1806 & 100 \\
\hline 13 a 19 & 650 & 91,7 & 59 & 8,3 & 709 & 100 \\
\hline 20 a 45 & 184 & 84,4 & 34 & 15,6 & 218 & 100 \\
\hline Total & 2571 & 94,1 & 162 & 5,9 & $2733^{*}$ & 100 \\
\hline
\end{tabular}

ECM = eventos cardíacos maiores; $\mathrm{N}=$ número de pacientes; $\left({ }^{*}\right)$ Excluídos pacientes que tiveram óbito, sem informação sobre insuficiência renal e ou sem seguimento clínico 
Tabela 37 - Distribuição dos pacientes da coorte B1 de acordo com as três faixas de risco (probabilidade logística) e pontuação obtida pelo escore DESIRE tardio

\begin{tabular}{|c|c|c|c|c|c|c|c|c|}
\hline \multirow[b]{3}{*}{ Probabilidade } & \multicolumn{8}{|c|}{ Escore simplificado (pontuação) } \\
\hline & \multicolumn{2}{|c|}{0 a 12} & \multicolumn{2}{|c|}{13 a 19} & \multicolumn{2}{|c|}{20 a 45} & \multicolumn{2}{|c|}{ Total } \\
\hline & $\mathbf{N}$ & $\%$ & $\mathbf{N}$ & $\%$ & $\mathbf{N}$ & $\%$ & $\mathbf{N}$ & $\%$ \\
\hline$\leq 6 \%$ & 1774 & 64,8 & 97 & 3,5 & 0 & 0 & 1871 & 68,3 \\
\hline$>6 \%-\leq 12 \%$ & 38 & 1,4 & 552 & 20,1 & 39 & 1,4 & 629 & 22,9 \\
\hline$>12 \%$ & 0 & 0 & 63 & 2,3 & 179 & 6,5 & 242 & 8,8 \\
\hline TOTAL & 1812 & 66,2 & 712 & 25,9 & 218 & 7,9 & $2742^{*}$ & 100 \\
\hline
\end{tabular}

$\mathrm{ECM}=$ eventos cardíacos maiores; $\mathrm{N}$ = número de pacientes; $\left({ }^{*}\right)$ Excluídos pacientes que tiveram óbito, sem informação sobre insuficiência renal e ou sem seguimento clínico

Na figura 18, encontra-se esquematizada a relação das variáveis clínicas e angiográficas, com sua respectiva pontuação, para o cálculo da probabilidade de ECM na evolução. 


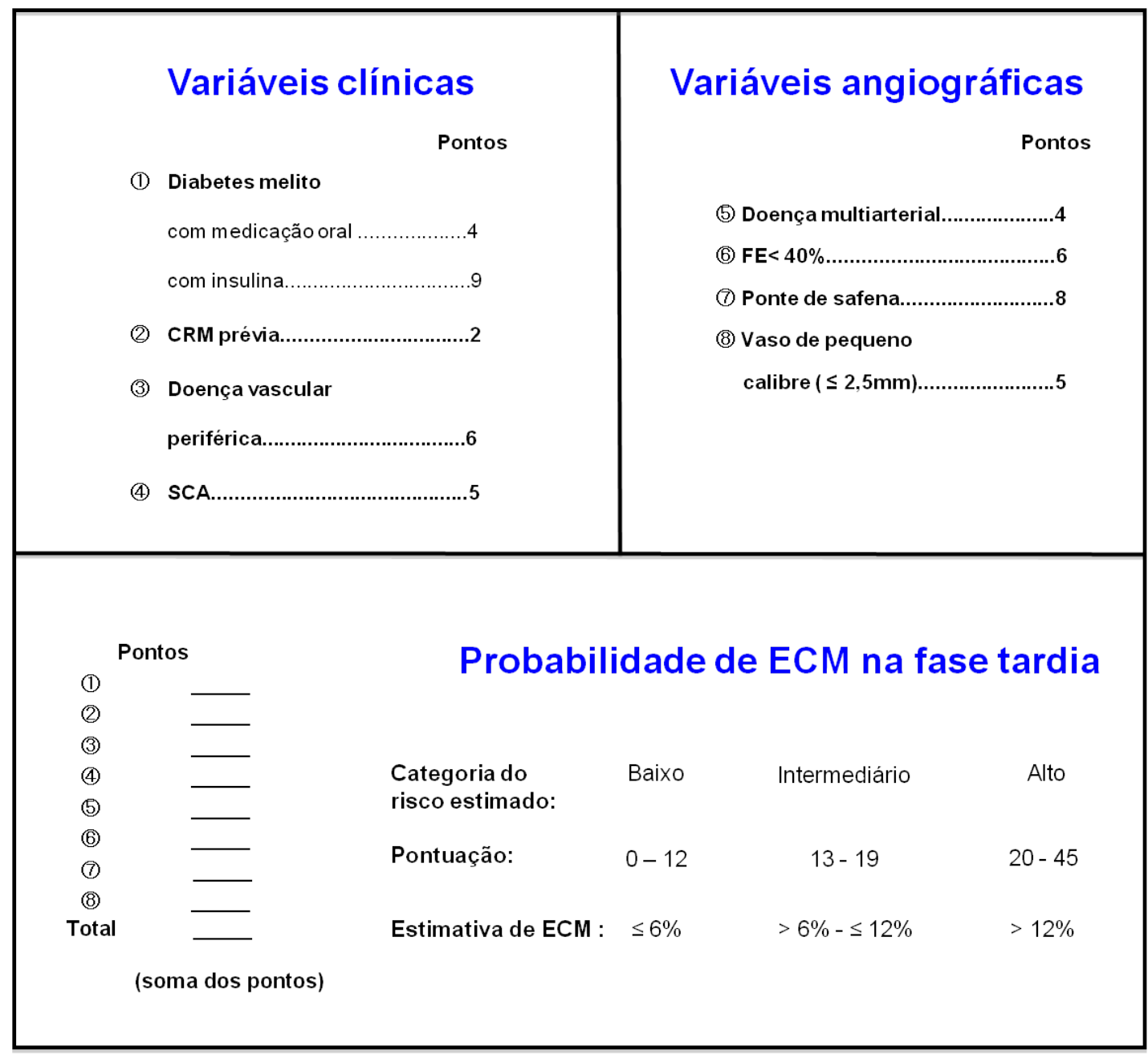

Figura 18 - Esquema do escore DESIRE para cálculo da probabilidade de ECM tardios. CRM = cirurgia de revascularização miocárdica; $E C M=$ eventos cardíacos maiores; FE = fração de ejeção; SCA = síndrome coronária aguda;

\subsubsection{Validação do escore DESIRE tardio}

Aplicamos o escore DESIRE tardio, nos pacientes da coorte B1 e B2, categorizando-os de acordo com suas respectivas faixas de risco para ECM na evolução. A correlação entre a ocorrência dos ECM nas coortes B1 e B2 
e as faixas de risco estimadas pelo escore DESIRE tardio é observada na figura 19 .

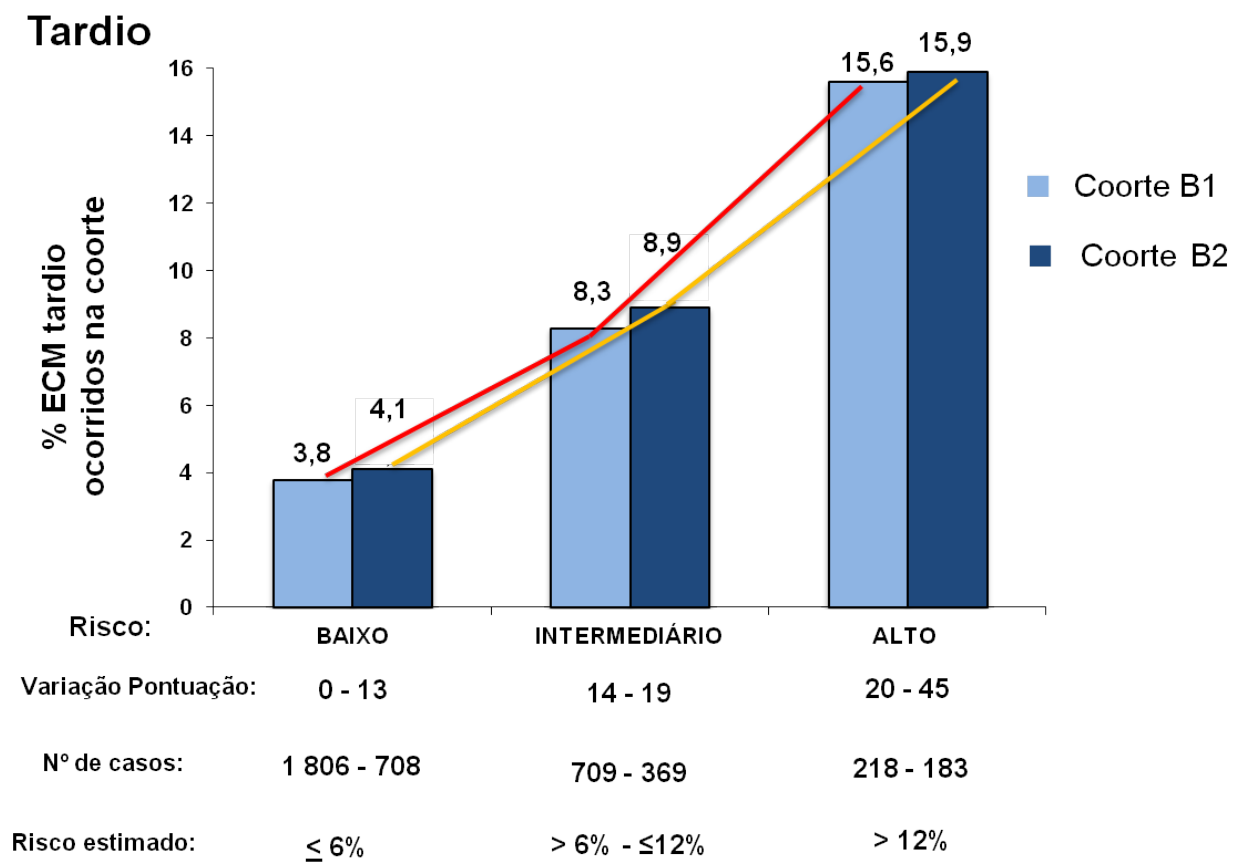

Figura 19 - Validação do escore DESIRE tardio. Correlação entre as probabilidades de risco esperadas para cada faixa de risco e os eventos observados nas coortes B1 e B2 (validação)

A acurácia do escore DESIRE tardio na coorte B2 foi de $0,63 \%$.

(Figura 20) A calibração do modelo, estimado pelo teste de HosmerLemeshow foi igual a 6,7 df8 $(p=0,56)$. 


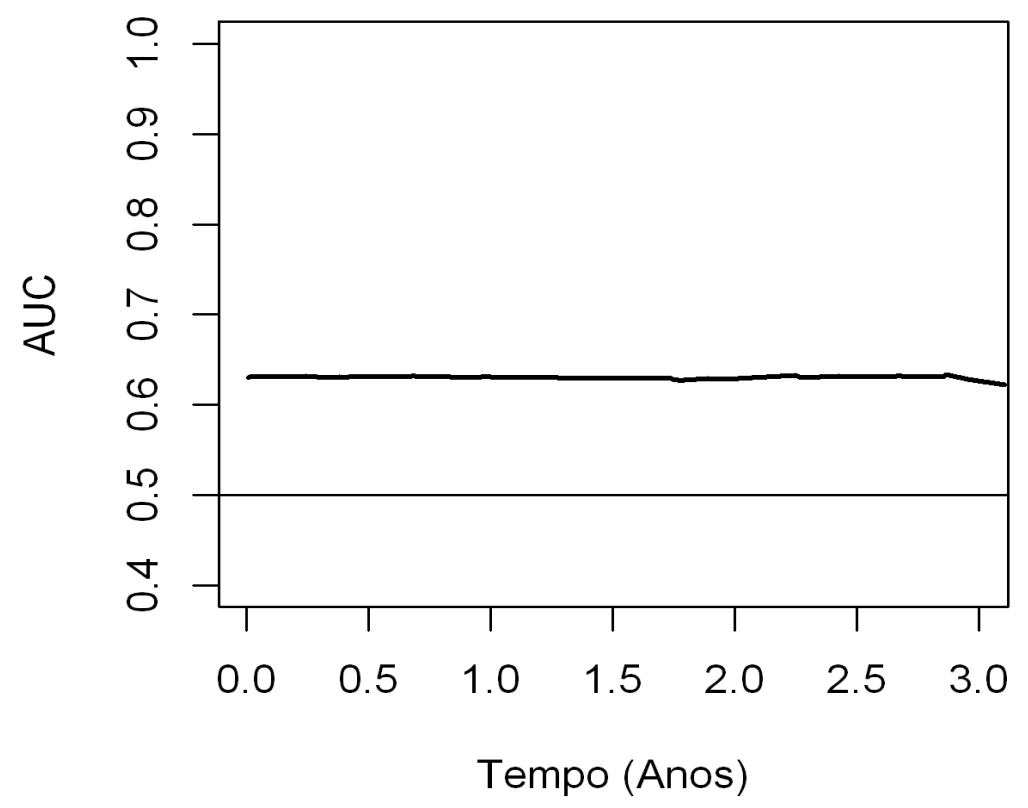

Figura 20 - Acurácia (AUC) do escore DESIRE tardio na coorte B2

\subsection{Resumo prático do escore DESIRE}

$\mathrm{Na}$ figura 21 consta um resumo prático dos resultados encontrados contendo as variáveis que compõem os dois modelos (hospitalar e tardio) e suas respectivas pontuações e probabildades estimadas de risco de ECM em ambas as fase. 


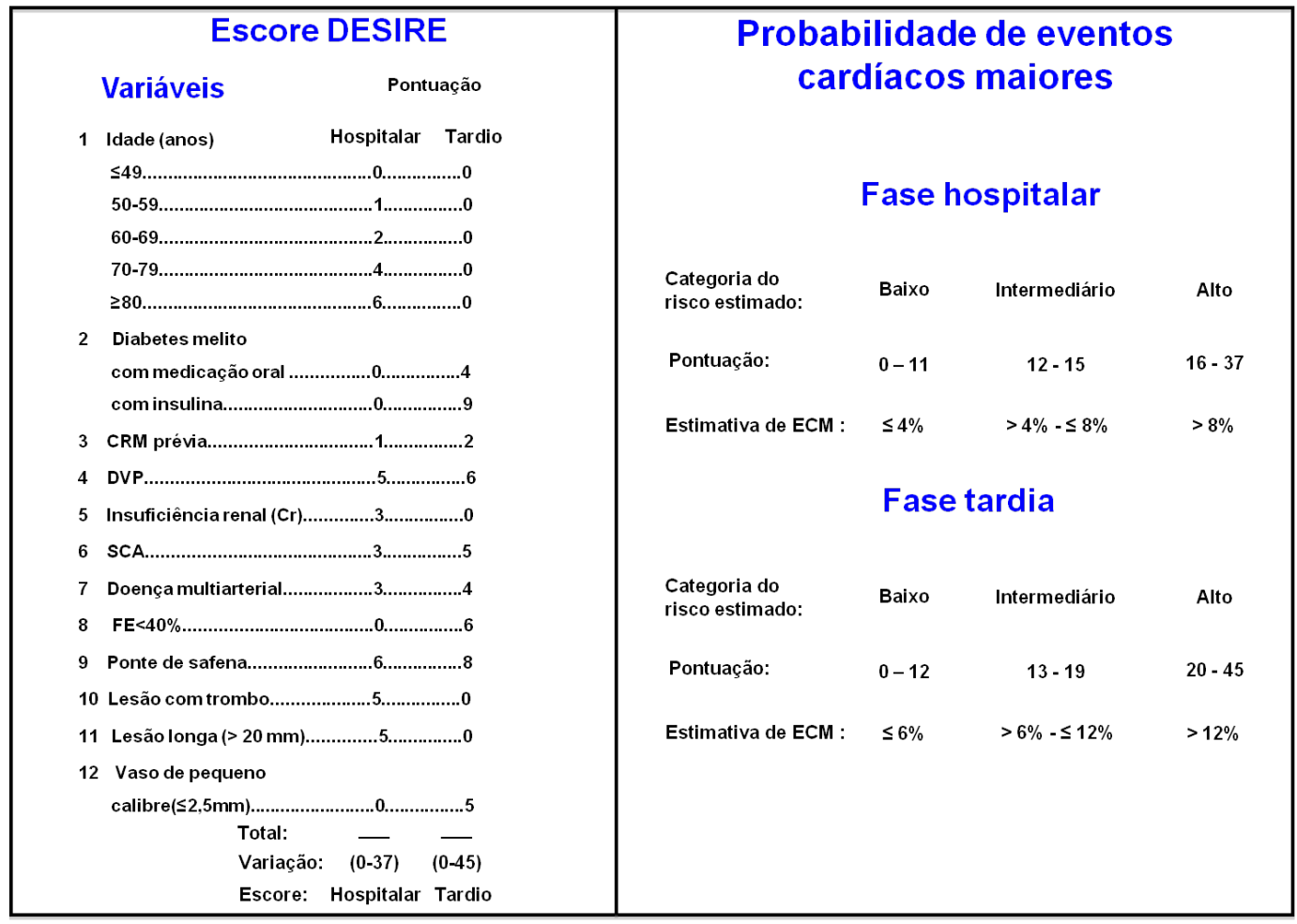

Figura 21- Resumo prático do escore DESIRE para as fases hospitalar e tardia 
5 DISCUSSÃO 
Até onde sabemos, o escore DESIRE é a primeira ferramenta de estratificação de risco pré-stents farmacológicos, desenvolvida e validada numa população brasileira, tratada contemporaneamente com estes instrumentais, e com seguimento clínico longo e praticamente completo.

Além disso, trata-se de um escore que contempla cerca de três dezenas de variáveis clínicas e angiográficas, refletindo de maneira abrangente cada caso e objetivando, não apenas a mortalidade, mas os três desfechos cardiovasculares maiores, com estimativas de risco categorizadas.

Destacam-se os principais achados desta investigação:

1. Na coorte B1 (de desenvolvimento), na fase hospitalar, identificamos nove variáveis (cinco clínicas e quatro angiográficas) que tiveram efeito prognóstico independente para ECM. Estas, portanto, compuseram o escore DESIRE hospitalar. Definindo-se três faixas de risco, cerca de um quarto dos casos exibiu risco moderado $(19,3 \%)$ ou alto $(8,6 \%)$. A acurácia do escore DESIRE hospitalar foi considerada muito boa: 0,74 .

2. Na coorte B2 (de validação), aplicando o escore DESIRE hospitalar foram identificados os três estratos de risco; sendo que $21,2 \%$ dos casos exibiam risco intermediário ou alto. 
3. Oito variáveis pré-ICP relacionaram-se na coorte $\mathrm{B} 1$, com os ECM na evolução e constituíram o escore DESIRE tardio. As três faixas de risco foram estabelecidas e um terço dos casos mostrou-se com risco intermediário $(25,9 \%)$ ou alto $(7,9 \%)$. A acurácia do escore DESIRE tardio foi considerada também satisfatória: 0,65 .

4. Em relação ao risco tardio, a coorte B2 exibiu elevação do risco, de 5,2 pontos porcentuais. A acurácia do escore tardio foi semelhante nas duas coortes.

\subsection{O Registro DESIRE}

No registro DESIRE estão incluídos consecutivamente todos os pacientes tratados com SF em nosso Serviço no HCor - ASS, desde sua liberação para uso clínico no País. Assim sendo, constitui-se numa excelente e dinâmica representação da prática rotineira da Cardiologia Intervencionista, para o tratamento contemporâneo da DAC.

Apesar de se caracterizar como um Registro dinâmico, incorporando, em uma década e meia de experiência, as evoluções técnicas, manteve extraordinária homogeneidade de resultados, consequência entre outros: 
1. do desempenho de operadores experientes, sempre os mesmos, ao longo de toda a série;

2. da busca incessante pelo apuro técnico, haja vista os resultados da angiografia e da ACQ pós-SF;

3. da rigorosa observância dos protocolos adjuntos e

4. da aplicação na coleta dos dados, com a preocupação de sua exatidão e completude, assim como na identificação e adjudicação minuciosa dos ECM. ${ }^{(10,54,57-68,100)}$

No Registro DESIRE, a população exibe, na apresentação, complexidade clínica e angiográfica pelo menos moderada. Destaquem-se na coorte B: a média das idades de $\geq 65$ anos; a prevalência considerável de diabéticos $(\approx 33 \%)$, de obesos $(\approx 30 \%)$ e de pacientes com pelo menos um procedimento de revascularização prévia (23\%); a presença não desprezível de insuficiência renal crônica ( $\approx 23 \%)$, de síndrome coronária aguda $(\approx 43 \%)$ e de doença multiarterial $(65 \%)$; o tratamento de lesões longas ( $\approx 52 \%)$, de vasos de pequeno calibre $(\approx 58 \%)$ e de lesões em bifurcação $(9,1 \%)$ e/ou do tipo B2/C (66,5\%). Mesmo com este perfil, foram observadas taxas elevadas de sucesso do procedimento $(99,7 \%)$ e de sucesso clínico $(95,3 \%)$, com ocorrência baixa de ECM na fase hospitalar $(4,1 \%)$, assim como no período evolutivo até dois anos (óbito cardíaco $=2,0 \%$; ECM acumulados $=10,4 \%$ ). Destaque-se o excepcional efeito anti-reestenóticodo desses instrumentais, com taxas de nova revascularização de 2,7\% e 4,3\% nas coortes B1 e B2, respectivamente. 
Na nossa experiência, percebemos nítida evolução de complexidade dos casos ao longo do tempo, facilmente identificada na comparação dessas coortes. Este fato denota por si só, a progressiva ampliação das indicações dos SF, nem sempre somente pela observância do indicado nas Diretrizes, mas antecipando-se a elas, como fruto da percepção dos bons resultados desses procedimentos, também em indicações off-label e em cenários mais desafiantes. ${ }^{(61)}$ Essa confiabilidade na revascularização percutânea atual foi sendo reforçada pela elevada taxa de sobrevida livre de ECM, detectada por nós, ao longo do tempo. ${ }^{(10)}$

Indiscutivelmentemente que o emprego sistemático de instrumentais avançados, equalizadores dos resultados, como nenhum dos precedentes, como é o caso dos SF contemporâneos, teve influência decisiva na excelência dos desfechos, mesmo numa população mais complexa como a coorte de validação. Um paralelo da literatura são as duas séries com expressivo número de pacientes, publicadas por Palmerini e cols. e Navarese e cols., que demonstraram a redução do risco de desfechos relacionados à segurança, como o infarto do miocárdio e a trombose protética, com emprego dos SF de segunda geração, quando comparados às outras tecnologias antecedentes. ${ }^{(115,116)}$

Outros Registros, com grande número de pacientes inseridos, serviram igualmente para o desenvolvimento de escores de risco na última década. ${ }^{(89)(92-94,117)}$ (Tabela 38). Percebe-se que, o óbito geral, até 30 dias desfecho mais frequentemente analisado, é de baixa ocorrência $(\leq 1,8 \%)$. Comparando as taxas de óbito hospitalar, nessas populações de 
desenvolvimento de escores, sobressai-se a ocorrência muito baixa de 0,34\% desse evento no Registro DESIRE hospitalar (coorte B1).

Enfatizamos que, diferentemente do escore DESIRE, a maior parte dos escores publicados deram ênfase apenas à evolução inicial (até 30 dias) e à analise de um único desfecho: o óbito geral. Além disso, foram desenvolvidos em populações tratadas com técnicas e instrumentais não contemporâneos, como por exemplo, o Registro de Toronto, no qual os SF representavam apenas $27 \%$ dos stents implantados. Isto tem grande significado, tanto é que mais recentemente, na série publicada por Kassaian e cols., com dados do National Cardiovascular Data Registry, embora tenha sido proposto o desenvolvimento de um escore de risco mais abrangente, estimando a taxa de ECM até um ano, o uso de stents não farmacológicos, em quase metade dos casos, foi um dos mais expressivos preditores da ocorrência desse desfecho. ${ }^{(118)}$ 
Tabela 38 - Registros clínicos utilizados para o desenvolvimento de escores de risco para estimativas de ECM após ICP na última década

\begin{tabular}{|c|c|c|c|c|c|}
\hline $\begin{array}{c}\text { Autor, } \\
\text { ano da } \\
\text { publicação }\end{array}$ & Registro & $\mathbf{N}$ & $\begin{array}{c}\text { População } \\
\text { estudada } \\
\text { (ano de } \\
\text { inclusão) }\end{array}$ & $\begin{array}{l}\text { Desfecho(s) } \\
\text { primários } \\
\text { analisados }\end{array}$ & $\begin{array}{c}\text { Fase } \\
\text { avaliada }\end{array}$ \\
\hline $\begin{array}{l}\text { Singh, }{ }^{(88)} \\
2007\end{array}$ & $\begin{array}{l}\text { Mayo Clinic } \\
\text { Registry }\end{array}$ & 7457 & $2000-2005$ & $\begin{array}{c}\text { óbito geral= } \\
1,8 \%\end{array}$ & Hospitalar \\
\hline $\begin{array}{c}\text { Chowdhary, }{ }^{(92)} \\
2009\end{array}$ & $\begin{array}{c}\text { University } \\
\text { Health Network } \\
\text { PCI Registry in } \\
\text { Toronto }\end{array}$ & 10694 & $2000-2008$ & $\begin{array}{c}\text { óbito geral= } \\
1,3 \%\end{array}$ & Hospitalar \\
\hline $\begin{array}{c}\text { Hamburguer, }{ }^{(93)} \\
2009\end{array}$ & $\begin{array}{l}\text { British } \\
\text { Columbia } \\
\text { Cardiac } \\
\text { Registry }\end{array}$ & 26350 & $2000-2004$ & $\begin{array}{c}\text { óbito geral= } \\
1,5 \%\end{array}$ & 30 dias \\
\hline $\begin{array}{l}\text { Peterson, }{ }^{(94)} \\
2010\end{array}$ & $\begin{array}{c}\text { National } \\
\text { Cardiovascular } \\
\text { Data Registry }\end{array}$ & 121183 & 2004-2006 & $\begin{array}{c}\text { óbito geral= } \\
1,24 \%\end{array}$ & 30 dias \\
\hline $\begin{array}{c}\text { Hannan }^{(96)} \\
2013\end{array}$ & $\begin{array}{c}\text { New York } \\
\text { State's } \\
\text { Percutaneous } \\
\text { Coronary } \\
\text { Intervention } \\
\text { System }\end{array}$ & 54223 & 2010 & $\begin{array}{c}\text { óbito geral = } \\
1,03 \%\end{array}$ & 30 dias \\
\hline $\begin{array}{c}\mathrm{Wu},{ }^{(117)} \\
2014\end{array}$ & $\begin{array}{l}\text { New York } \\
\text { State's } \\
\text { Percutaneous } \\
\text { Coronary } \\
\text { Intervention } \\
\text { System }\end{array}$ & 11897 & 2003-2008 & $\begin{array}{c}\text { óbito geral: } \\
1 \text { ano }(4,8 \%) \\
3 \text { anos }(9,8 \%) \\
5 \text { anos } \\
(16,1 \%)\end{array}$ & até 5 anos \\
\hline $\begin{array}{c}\text { Moreira, } \\
2017\end{array}$ & $\begin{array}{c}\text { Registro } \\
\text { DESIRE } \\
\text { (Coorte B1) }\end{array}$ & 2863 & $2007-2012$ & $\begin{array}{c}\mathrm{ECM}^{* *} \\
\text { hospitalares } \\
(3,8 \%) \\
\text { tardios }(6,3 \%)\end{array}$ & $\begin{array}{l}\text { Hospitalar } \\
\text { e tardia } \\
\text { até } 2 \text { anos }\end{array}$ \\
\hline
\end{tabular}

Definições: $\left(^{*}\right)$ ECM (Mayo Clinic)= óbito geral, IM com onda "Q", CRM urgência/ emergência e AVE; $\left(^{* *}\right)$ ECM (DESIRE)= óbito cardíaco, IM (elevação da CK-MB massa $\geq 5$ vezes do percentil 99) e RLA 
Sendo, portanto, os Registros disponíveis diferentes nos seus escopos e desfechos apurados, e com adoção de definições distintas, a comparabilidade oferecida na literatura é escassa e precária, geralmente não alinhada no tempo.

\subsection{0 escore DESIRE}

Os dois modelos de regressão logística propostos permitiram uma muito boa estimativa de risco contendo: $1^{\circ}$ - um desfecho composto (ECM) que inclui os dois grandes eventos que traduzem segurança (óbito cardíaco e infarto não fatal) e o terceiro que se refere à efetividade da ICP (revascularização da lesão-alvo). $2^{\circ}$ - Não só isso mas também, abordando com as devidas diferenças as duas fases de seguimento clínico pós-SF: hospitalar e tardia, numa população que reflete com grande fidelidade a prática dos dias atuais.

Desta forma, o escore em questão oferece informações prognósticas mais robustas e adicionais, em comparação àquelas provenientes dos modelos anteriormente desenvolvidos e já discutidos. 
Além disso, o escore DESIRE foi considerado uma ferramenta amigável, na prática, por conter variáveis de fácil obtenção e bem definidas, evitando desta forma, a variação entre observadores. Alguns aspectos reforçam sua aplicabilidade, dentre eles, como já destacada, a própria contemporaneidade da casuística.

Outro aspecto de realce é que foram desenvolvidos, ao invés de um, dois escores: hospitalar e tardio, em decorrência da verificação que as variáveis preditoras independentes de prognóstico não são idênticas, para as duas fases. Cinco variáveis são coincidentes, porém, na fase hospitalar, há outras quatro que influenciam os desfechos, mas não apresentam impacto independente na evolução tardia. Já no período após a alta, três condições, ainda que não tenham efeito independente na fase hospitalar, mostraram-se preditoras de prognóstico na evolução. (Figura 22)

\begin{tabular}{|c|c|c|}
\hline Variáveis & Hospitalar & Evolutiva \\
\hline Coincidentes & $\begin{array}{r}\text { Cirurgia de revascularização miocárdica prévia } \\
\text { Doença vascular periférica } \\
\text { Síndrome coronária aguda } \\
\text { Doença multiarterial } \\
\text { Lesão em ponte de safena }\end{array}$ \\
\hline Não coincidentes & $\begin{array}{c}\text { Insuficiência renal (Cr) } \\
\text { Lesão com trombo } \\
\text { Lesão longa }\end{array}$ & $\begin{array}{c}\text { Diabete melito } \\
\text { FE < }<40 \%\end{array}$ \\
& Lesão em vaso pequeno calibre \\
\hline
\end{tabular}

Figura 22 - Registro DESIRE: preditores independentes de ECM pós-SF nas fases hospitalar e tardia pós-alta. 
A não perfeita coincidência das variáveis que têm impacto na fase de internação e na evolutiva é decorrente do fato de que o que influencia na fase hospitalar é distinto do que influencia na evolução. Os ECM na fase hospitalar têm, em geral, relação com a apresentação clínica e com o procedimento em si, enquanto que os desfechos na evolução tardia têm a ver com as histórias naturais do procedimento e da própria DAC.

$\mathrm{Na}$ confecção do escore DESIRE hospitalar, adotamos para o diagnóstico de IM periprocedimento, a elevação da CKMB massa como marcador de necrose. Vale ressaltar que o diagnóstico do infarto do miocárdio tipo $4 a$ ainda é motivo de grandes questionamentos, tendo passado por modificações, inclusive na última década. ${ }^{(119-122)}$. Há dúvidas, não só quanto ao marcador de necrose miocárdica a ser considerado, mas igualmente quanto à magnitude da elevação do mesmo ${ }^{(109)}$ e também se existe correlação entre a ocorrência isolada destas elevações, em pacientes assintomáticos e sem complicações no procedimento. ${ }^{(123,124)}$

As recomendações mais recentes tentam evitar a excessiva valorização das elevações dos marcadores de necrose miocárdica pós-ICP, que muitas vezes ocorrem sem de fato ter implicações prognósticas ${ }^{(122)}$,em relação à ocorrência de óbito e de revascularização da lesão-alvo. Foi isso, portanto, que levamos em consideração nas nossas análises, valorizando menos as pequenas ou isoladas elevações destes marcadores, e mais as elevações acentuadas ( $\geq 5$ vezes o porcentil 99 ), sobretudo se associadas a outros eventos, como: dor típica; alterações do ECG pós-ICP; anormalidades do fluxo coronário, ao final do implante etc. 
Assim sendo, no escore DESIRE, a CKMB massa, especialmente quando considerado o valor de 5 vezes acima do percentil 99, selecionou os pacientes que de fato tiveram sua evolução clínica modificada. Na coorte B1, por exemplo, os pacientes que apresentaram elevação da CKMB massa 5 vezes acima do percentil 99 , cursaram com quase $60 \%$ a mais de ECM no seguimento.

Wu e cols. ${ }^{(99)}$ também desenvolveram escores distintos para as fases hospitalar ${ }^{(99)}$ e tardia ${ }^{(117)}$, entretanto o fizeram em populações diferentes. $O$ primeiro, publicado em 2006 foi desenvolvido para estimar a mortalidade hospitalar, a partir dos dados de pacientes tratados em 2002 e, como já foi mencionado, com o uso de stents não famacológicos. O segundo, para acessar somente o risco de óbito tardio (até cinco anos), que se baseou em um modelo de riscos proporcionais de Cox (assim como no escore DESIRE), identificando doze variáveis no modelo final, associadas à mortalidade no longo prazo, sem contudo destacar as angiográficas, que sabidamente exercem papel prognóstico importante.

Outras iniciativas de avaliar probabilidades de risco no seguimento tardio não podem ser aqui utilizadas, como comparação ao escore DESIRE, por que: ou se limitaram a avaliar apenas um desfecho clínico (óbito); ou partiram de populações não atuais, ou ainda restringiram-se somente a determinados subgrupos na evolução pós-ICP(125,126).

Em 2007, Singh e cols. ${ }^{(88)}$ publicaram o escore de risco Mayo Clinic, um dos poucos na literatura a avaliar não apenas a ocorrência óbito, mas também de ECM, entretanto apenas na fase hospitalar. Além disso, a 
definição de ECM é bem distinta daquela utilizada no registro DESIRE, limitando qualquer possibilidade de análise comparativa. Adicionalmente, esse modelo de predição incluiu apenas variáveis clínicas e laboratoriais, desconsiderando os dados da coronariografia. Já o escore do North West Quality Improvement Programme in Cardiac interventions (WQIP) ${ }^{(127)}$ incluiu, na sua construção variáveis angiográficas para análise dos mesmos ECM que o escore da Mayo Clinic, e de fato, variáveis angiográficas destacaramse com expressiva contribuição na estimativa de risco.

A importância das variáveis angiográficas é percebida sobretudo pelo seu efeito na predição da revascularização da lesão-alvo. Esse desfecho clínico tem baixa prevalência na fase hospitalar, decorrente em especial das elevadas taxas de sucesso do procedimento e, também das baixas ocorrências de trombose protética, tornando as reintervenções na lesão-alvo de ocorrência rara. Já na fase evolutiva, esse desfecho tem maior representatividade, pois traduz inicialmente a reestenose intrastent $e$, mais adiante no tempo, a progressão da DAC.

Na era da ATC com balão, por exemplo, o modelo de risco baseado exclusivamente na caracterização das lesões pela classificação do American College of Cardiology/American Heart Association (ACC/AHA) definiu onze variáveis angiográficas correlacionadas à ocorrência do desfecho clínico combinado: óbito, infarto do miocárdio, CRM de urgência e insucesso do procedimento. Atualmente, diante do maior perfil de segurança das ICP, a CRM de urgência tornou-se um evento clínico raríssimo e, por isso, deixou de ser considerado nos modelos atuais. ${ }^{(16)}$ Outra limitação desse escore, 
que foi amplamente utilizado no passado, é a grande variabilidade entre observadores.

Na fase atual dos SF, o escore $\operatorname{SYNTAX}^{(84,128)}$ foi desenvolvido para oferecer uma avaliação objetiva do grau e da extensão da DAC, inclusive considerando a caracterização das lesões no estudo homônimo, objetivando a comparação entre CRM e ICP, em pacientes com maior complexidade angiográfica (doença multiarterial e/ou lesões no TCE). Logo se percebeu sua habilidade em discriminar ECM após ICP, o que o colocou mais na prática da Cardiologia Intervencionista, apesar de ter sido considerado também para indicar prognóstico pós-CRM. ${ }^{(128)}$ Por outro lado, considerar que pacientes com a mesma pontuação têm evolução clínica semelhante, a despeito de diferentes características clínicas, contrapõe evidências já muito bem definidas. ${ }^{(91)}$

O mesmo grupo de pesquisadores, desenvolveu então o escore SYNTAX II, agregando sete variáveis clínicas ao cálculo do escore SYNTAX angiográfico. Mas, até o presente avaliaram apenas a mortalidade no longo prazo. ${ }^{(97)}$ Além disso, para o cálculo das estimativas de risco, é necessário o adequado treinamento para a utilização do aplicativo do SYNTAX anatômico, que não é tão amigável. 


\subsection{Considerações sobre a acurácia do escore DESIRE}

A acurácia do escore DESIRE em ambas as fases foi considerada boa $(\sim 0,70 \%)$ e robusta, mas não excelente, especialmente quando comparada a de outros escores de execução mais complexa, como o escore de Nova York, que exibe acurácia de de 0,88\%. Esses achados podem ser parcialmente explicados pelo fato de que o escore DESIRE é mais abrangente, estimando o risco para ECM ao invés de um único componente como o óbito, como observado na maioria dos outros Registros.

Além disso, a evolução contínua das indicações, dispositivos e técnicas do ICP tende a enfraquecer a reprodutibilidade dos escores desenvolvidos e validados em diferentes momentos. Como exemplo, cerca de $50 \%$ dos SF utilizados na fase de desenvolvimento do DESIRE ainda eram da primeira geração, enquanto que praticamente nenhum destes dispositivos foi utilizado na fase de validação.

Por outro lado, a complexidade observada na segunda fase foi consideravelmente maior do que na coorte de desenvolvimento. 


\subsection{Limitações}

A principal limitação de nosso escore é a falta de validação externa, pois representa uma análise de centro único. Portanto, a extrapolação de nossos achados para outras experiências deve ser vista com cautela. No entanto, vale ressaltar que a maioria das variáveis incluídas no modelo estatístico para construir a pontuação do escore de risco DESIRE são tradicionalmente associadas a resultados negativos em outras populações com DAC tratadas com SF. 
6 CONCLUSÕES 
O escore DESIRE subdividido em duas fases, hospitalar e tardia, constitui-se numa ferramenta eficaz e amigável para estimativa do risco global em população de pacientes revascularizados percutaneamente com SF contemporâneos. Isto fica patente, considerando-se a precisão da antecipação de eventos que vieram a ocorrer

1. As variáveis que compõem o escore DESIRE hospitalar são: cinco variáveis clínicas (idade, CRM prévia, síndrome coronária aguda, doença vascular periférica e insuficiência renal) e quatro angiográficas (doença multiarterial, ponte de safena, lesão com trombo e lesão longa).

2. O escore DESIRE tardio é composto por oito variáveis sendo quatro clínicas (Diabete melito, CRM prévia, doença vascular periférica e síndrome coronária aguda) e quatro angiográficas (doença multiarterial, fração de ejeção, ponte de safena e lesão em vaso de pequeno calibre).

3. A partir da aplicação do escore DESIRE, obtendo-se a pontuação individual, estimou-se a probabilidade de ECM assim categorizadas para as duas fases:

a) Hospitalar - baixo risco com probabilidade de ECM $\leq$ $4 \%$; risco intermediário, $>4 \%$ e $\leq 8 \%$ e alto $>8 \%$;

b) Tardia - baixo risco $\leq 6 \%$; risco intermediário $>6 \%$ e $\leq$ $12 \%$ e alto $->12 \%$.

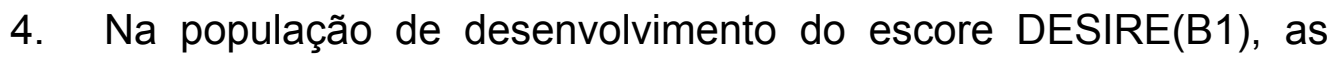
taxas de óbito cardíaco, infarto do miocárdio e revascularização 
da lesão-alvo são baixas em ambas as fases: 0,24\% (N=7P), $3,6 \%(N=110 P)$ e $0,0 \%$ na fase hospitalar; $2,0 \%(N=57 P), 1,6 \%$ $(\mathrm{N}=46 \mathrm{P})$ e $2,7 \%(\mathrm{~N}=76 \mathrm{P})$ na evolução.

5. Na coorte B2, na qual o escore foi validado, as taxas de óbito cardíaco, infarto do miocárdio e revascularização da lesão-alvo são igualmente baixas $0,25 \%(N=3 P), 4,4 \%(N=53 P)$ e $0,0 \%$ na fase hospitalar; $2,2 \%(N=26 P), 2,4 \%(N=28 P)$ e $4,3 \%(N=91 P)$ na tardia. 
7 REFERÊNCIAS BIBLIOGRÁFICAS 


\section{Referências Bibliográficas}

1.Azambuja MIR, Foppa M, Maranhão MFdC, Achutti AC. Impacto econômico dos casos de doença cardiovascular grave no Brasil: uma estimativa baseada em dados secundários. Arquivos Brasileiros de Cardiologia. 2008;91:163-71.

2.Global, regional, and national life expectancy, all-cause mortality, and cause-specifi c mortality for 249 causes of death, 1980-2015: a systematic analysis for the Global Burden of Disease Study 2015. lancet. 2016;2016(388).

3.Souza MdFMd, Alencar AP, Malta DC, Moura L, Mansur AdP. Análise de séries temporais da mortalidade por doenças isquêmicas do coração e cerebrovasculares, nas cinco regiões do Brasil, no período de 1981 a 2001. Arquivos Brasileiros de Cardiologia. 2006;87:735-40.

4.Mansur AdP, Favarato D. Mortalidade por doenças cardiovasculares no Brasil e na região metropolitana de São Paulo: atualização 2011. Arquivos Brasileiros de Cardiologia. 2012;99:755-61.

5.Writing Group M, Mozaffarian D, Benjamin EJ, Go AS, Arnett DK, Blaha MJ, et al. Heart Disease and Stroke Statistics-2016 Update: A Report From the American Heart Association. Circulation. 2016;133(4):e38-360.

6. Mansur AdP, Favarato D. Trends in Mortality Rate from Cardiovascular Disease in Brazil, 1980-2012. Arquivos Brasileiros de Cardiologia. 2016;107:20-5.

7.Malta DC, Moura Ld, Prado RRd, Escalante JC, Schmidt MI, Duncan BB. Mortalidade por doenças crônicas não transmissíveis no Brasil e suas 
regiões, 2000 a 2011. Epidemiologia e Serviços de Saúde. 2014;23(4):599608.

8. Teich V AD. Estimativa de Custo da Síndrome Coronariana Aguda no Brasil. Rev Bras Cardiol. 2011;24(2):85-94.

9.Gruntzig AR, Senning A, Siegenthaler WE. Nonoperative dilatation of coronary-artery stenosis: percutaneous transluminal coronary angioplasty. $\mathrm{N}$ Engl J Med. 1979;301(2):61-8.

10.Costa JR, Jr., Sousa A, Moreira AC, Costa RA, Cano M, Maldonado G, et al. Incidence and predictors of very late ( $>$ or $=4$ years) major cardiac adverse events in the DESIRE (Drug-Eluting Stents in the Real World)-Late registry. JACC Cardiovasc Interv. 2010;3(1):12-8.

11.Guimaraes JI, Sousa JE, Ribeiro E, Mattos LA, Sousa AG, Nunes GL, et al. [Guidelines for the indications and use of percutaneous interventions and intracoronary stent in clinical practice]. Arq Bras Cardiol. 2003;80 Suppl 1:114.

12.Colombo A, Hall P, Nakamura S, Almagor Y, Maiello L, Martini G, et al. Intracoronary stenting without anticoagulation accomplished with intravascular ultrasound guidance. Circulation. 1995;91(6):1676-88.

13.Leon MB, Baim DS, Popma JJ, Gordon PC, Cutlip DE, Ho KK, et al. A clinical trial comparing three antithrombotic-drug regimens after coronaryartery stenting. Stent Anticoagulation Restenosis Study Investigators. N Engl J Med. 1998;339(23):1665-71.

14.Sousa AG, Mattos LA, Campos Neto Cde M, Carvalho HG, Stella FP, Nunes G. [Percutaneous myocardial revascularization procedures in Brazil in 
1996-1997 compared to the 1992-1993 period. A report of the National Registry--National Center for Cardiovascular Interventions (CENIC)]. Arq Bras Cardiol. 1998;70(6):423-30.

15.Cutlip DE, Chauhan MS, Baim DS, Ho KK, Popma JJ, Carrozza JP, et al. Clinical restenosis after coronary stenting: perspectives from multicenter clinical trials. J Am Coll Cardiol. 2002;40(12):2082-9.

16.Garg S, Serruys PW. Coronary stents: current status. J Am Coll Cardiol. 2010;56(10 Suppl):S1-42.

17.Rezende CA, Luchesi C, de Lourdes PBarbo M, Duek EA. Membranas de poli (ácido lático-co-ácido glicólico) como curativos para pele: degradação in vitro e in vivo. POLIMEROS CIENCIA E TECNOLOGIA. 2005;15(3):232.

18.Windecker S, Serruys PW, Wandel S, Buszman P, Trznadel S, Linke A, et al. Biolimus-eluting stent with biodegradable polymer versus sirolimuseluting stent with durable polymer for coronary revascularisation (LEADERS): a randomised non-inferiority trial. Lancet. 2008;372(9644):1163-73.

19.Serruys PW, Garg S, Abizaid A, Ormiston J, Windecker S, Verheye S, et al. A randomised comparison of novolimus-eluting and zotarolimus-eluting coronary stents: 9-month follow-up results of the EXCELLA II study. Eurolntervention. 2010;6(2):195-205.

20.Grundeken MJ, Wykrzykowska JJ, Ishibashi Y, Garg S, de Vries T, Garcia-Garcia HM, et al. First generation versus second generation drugeluting stents for the treatment of bifurcations: 5-year follow-up of the LEADERS all-comers randomized trial. Catheter Cardiovasc Interv. 2016;87(7):E248-60. 
21.Grube E, Chevalier B, Smits P, Dzavik V, Patel TM, Mullasari AS, et al. The SPIRIT $V$ study: a clinical evaluation of the XIENCE $V$ everolimuseluting coronary stent system in the treatment of patients with de novo coronary artery lesions. JACC Cardiovasc Interv. 2011;4(2):168-75.

22.Krucoff MW, Rutledge DR, Gruberg L, Jonnavithula L, Katopodis JN, Lombardi $W$, et al. A new era of prospective real-world safety evaluation primary report of XIENCE V USA (XIENCE V Everolimus Eluting Coronary Stent System condition-of-approval post-market study). JACC CardiovasC Interv. 2011;4(12):1298-309.

23. Hannan EL, Zhong Y, Wu C, Walford G, Holmes DR, Jr., Jacobs AK, et al. Everolimus-eluting stents and zotarolimus-eluting stents for percutaneous coronary interventions: two-year outcomes in New York State. Catheter Cardiovasc Interv. 2013;81(7):1097-105.

24.Onuma Y, Piazza N, Ormiston JA, Serruys PW. Everolimus-eluting bioabsorbable stent--Abbot Vascular programme. Eurolntervention. 2009;5 Suppl F:F98-F102.

25.Kaul U, Patel TM, Zambahari R, Mullasari AS, Bahl VK, Stuteville M, et al. Evaluation of the XIENCE $V$ everolimus eluting coronary stent system in the Asian population of the SPIRIT $V$ single arm study. 2-year clinical follow-up data. Indian Heart J. 2011;63(5):402-8.

26.Sousa JE, Costa MA, Abizaid A, Abizaid AS, Feres F, Pinto IM, et al. Lack of neointimal proliferation after implantation of sirolimus-coated stents in human coronary arteries: a quantitative coronary angiography and threedimensional intravascular ultrasound study. Circulation. 2001;103(2):192-5. 
27.Sousa JE, Costa MA, Abizaid A, Feres F, Seixas AC, Tanajura LF, et al. Four-year angiographic and intravascular ultrasound follow-up of patients treated with sirolimus-eluting stents. Circulation. 2005;111(18):2326-9.

28.Regar E, Serruys PW. [The Ravel trial. Zero percent restenosis: A cardiologists dream comes true!]. Rev Esp Cardiol. 2002;55(5):459-62.

29.Morice MC, Serruys PW, Sousa JE, Fajadet J, Ban Hayashi E, Perin M, et al. A randomized comparison of a sirolimus-eluting stent with a standard stent for coronary revascularization. N Engl J Med. 2002;346(23):1773-80.

30.Moses JW, Leon MB, Popma JJ, Fitzgerald PJ, Holmes DR, O'Shaughnessy $\mathrm{C}$, et al. Sirolimus-eluting stents versus standard stents in patients with stenosis in a native coronary artery. The New England journal of medicine. 2003;349(14):1315-23.

31.Schampaert E, Cohen EA, Schluter M, Reeves F, Traboulsi M, Title LM, et al. The Canadian study of the sirolimus-eluting stent in the treatment of patients with long de novo lesions in small native coronary arteries (CSIRIUS). J Am Coll Cardiol. 2004;43(6):1110-5.

32.Schofer J, Schluter M, Gershlick AH, Wijns W, Garcia E, Schampaert E, et al. Sirolimus-eluting stents for treatment of patients with long atherosclerotic lesions in small coronary arteries: double-blind, randomised controlled trial (E-SIRIUS). Lancet. 2003;362(9390):1093-9.

33.Grube E, Silber S, Hauptmann KE, Mueller R, Buellesfeld L, Gerckens U, et al. TAXUS I: six- and twelve-month results from a randomized, doubleblind trial on a slow-release paclitaxel-eluting stent for de novo coronary lesions. Circulation. 2003;107(1):38-42. 
34.Silber S, Colombo A, Banning AP, Hauptmann K, Drzewiecki J, Grube E, et al. Final 5-year results of the TAXUS II trial: a randomized study to assess the effectiveness of slow- and moderate-release polymer-based paclitaxeleluting stents for de novo coronary artery lesions. Circulation. 2009;120(15):1498-504.

35. Tanabe K, Serruys PW, Grube E, Smits PC, Selbach G, van der Giessen WJ, et al. TAXUS III Trial: in-stent restenosis treated with stent-based delivery of paclitaxel incorporated in a slow-release polymer formulation. Circulation. 2003;107(4):559-64.

36.Ellis SG, Stone GW, Cox DA, Hermiller J, O'Shaughnessy C, Mann T, et al. Long-term safety and efficacy with paclitaxel-eluting stents: 5-year final results of the TAXUS IV clinical trial (TAXUS IV-SR: Treatment of De Novo Coronary Disease Using a Single Paclitaxel-Eluting Stent). JACC CardiovasC Interv. 2009;2(12):1248-59.

37.Stone GW, Ellis SG, O'Shaughnessy CD, Martin SL, Satler L, McGarry T, et al. Paclitaxel-eluting stents vs vascular brachytherapy for in-stent restenosis within bare-metal stents: the TAXUS V ISR randomized trial. JAMA. 2006;295(11):1253-63.

38.Grube E, Dawkins K, Guagliumi G, Banning A, Zmudka K, Colombo A, et al. TAXUS VI final 5-year results: a multicentre, randomised trial comparing polymer-based moderate-release paclitaxel-eluting stent with a bare metal stent for treatment of long, complex coronary artery lesions. Eurolntervention. 2009;4(5):572-7.

39.Dawkins KD, Grube E, Guagliumi G, Banning AP, Zmudka K, Colombo A, et al. Clinical efficacy of polymer-based paclitaxel-eluting stents in the treatment of complex, long coronary artery lesions from a multicenter, 
randomized trial: support for the use of drug-eluting stents in contemporary clinical practice. Circulation. 2005;112(21):3306-13.

40.Sousa JE, Serruys PW, Costa MA. New frontiers in cardiology: drugeluting stents: Part I. Circulation. 2003;107(17):2274-9.

41.Sousa JE, Serruys PW, Costa MA. New frontiers in cardiology: drugeluting stents: Part II. Circulation. 2003;107(18):2383-9.

42.Serruys PW. Fourth annual American College of Cardiology international lecture: a journey in the interventional field. J Am Coll Cardiol. 2006;47(9):1754-68.

43.Daemen J, Serruys PW. Drug-eluting stent update 2007: part I. A survey of current and future generation drug-eluting stents: meaningful advances or more of the same? Circulation. 2007;116(3):316-28.

44.Daemen J, Serruys PW. Drug-eluting stent update 2007: part II: Unsettled issues. Circulation. 2007;116(8):961-8.

45.Stone GW, Moses JW, Ellis SG, Schofer J, Dawkins KD, Morice MC, et al. Safety and efficacy of sirolimus- and paclitaxel-eluting coronary stents. $\mathrm{N}$ Engl J Med. 2007;356(10):998-1008.

46.Wykrzykowska JJ, Raber L, de Vries T, Bressers M, Buszman P, Linke A, et al. Biolimus-eluting biodegradable polymer versus sirolimus-eluting permanent polymer stent performance in long lesions: results from the LEADERS multicentre trial substudy. Eurolntervention. 2009;5(3):310-7.

47.Han Y, Jing Q, Xu B, Yang L, Liu H, Shang X, et al. Safety and efficacy of biodegradable polymer-coated sirolimus-eluting stents in "real-world" 
practice: 18-month clinical and 9-month angiographic outcomes. JACC Cardiovasc Interv. 2009;2(4):303-9.

48.Lemos PA, Serruys PW, van Domburg RT, Saia F, Arampatzis CA, Hoye $A$, et al. Unrestricted utilization of sirolimus-eluting stents compared with conventional bare stent implantation in the "real world": the RapamycinEluting Stent Evaluated At Rotterdam Cardiology Hospital (RESEARCH) registry. Circulation. 2004;109(2):190-5.

49.Urban P, Gershlick AH, Guagliumi G, Guyon P, Lotan C, Schofer J, et al. Safety of coronary sirolimus-eluting stents in daily clinical practice: one-year follow-up of the e-Cypher registry. Circulation. 2006;113(11):1434-41.

50.Marroquin OC, Selzer F, Mulukutla SR, Williams DO, Vlachos HA, Wilensky RL, et al. A comparison of bare-metal and drug-eluting stents for off-label indications. N Engl J Med. 2008;358(4):342-52.

51.Applegate RJ, Sacrinty MT, Kutcher MA, Santos RM, Gandhi SK, Baki TT, et al. "Off-label" stent therapy 2-year comparison of drug-eluting versus bare-metal stents. J Am Coll Cardiol. 2008;51(6):607-14.

52.Kirtane AJ, Gupta A, lyengar S, Moses JW, Leon MB, Applegate R, et al. Safety and efficacy of drug-eluting and bare metal stents: comprehensive meta-analysis of randomized trials and observational studies. Circulation. 2009;119(25):3198-206.

53.Lagerqvist B, James SK, Stenestrand U, Lindback J, Nilsson T, Wallentin $\mathrm{L}$, et al. Long-term outcomes with drug-eluting stents versus bare-metal stents in Sweden. N Engl J Med. 2007;356(10):1009-19. 
54.Sousa AGdMR, Costa Junior JR, Moreira A, Costa RAd, Cano MN, Andrade GAM, et al. Evolução clínica tardia dos stents farmacológicos: segurança e eficácia até cinco anos do Registro DESIRE. Revista Brasileira de Cardiologia Invasiva. 2007;15:221-7.

55. Quizhpe AR, Feres F, de Ribamar Costa J, Jr., Abizaid A, Maldonado G, Costa $R$, et al. Drug-eluting stents vs bare metal stents for the treatment of large coronary vessels. Am Heart J. 2007;154(2):373-8.

56.Sousa A, Costa JR, Jr., Moreira AC, Cano M, Maldonado G, Costa RA, et al. Long-term clinical outcomes of the Drug-Eluting Stents in the Real World (DESIRE) Registry. J Interv Cardiol. 2008;21(4):307-14.

57.Costa JR, Jr., Sousa A, Moreira AC, Costa RA, Maldonado G, Cano MN, et al. Drug-eluting stents in the elderly: long-term (> one year) clinical outcomes of octogenarians in the DESIRE (Drug-Eluting Stents In the REal world) registry. J Invasive Cardiol. 2008;20(8):404-10.

58.Mendes A, Sousa AGMR, Costa RA, Moreira A, Costa Jr. JR, Maldonado $\mathrm{G}$, et al. Impacto dos stents farmacológicos no tratamento percutâneo de lesões coronárias em bifurcação: resultados clínicos tardios de um estudo comparativo incluindo pacientes do "mundo-real". Revista Brasileira de Cardiologia Invasiva. 2008;16:406-14.

59.Moreira AC, Sousa AGMR, Costa Jr. JdR, Costa RA, Maldonado GA, Cano MN, et al. Evolução tardia após intervenção coronária percutânea com stents farmacológicos em pacientes diabéticos do Registro DESIRE (DrugEluting Stents In the REal world). Revista Brasileira de Cardiologia Invasiva. 2008;16:185-92. 
60.Costa RA, Sousa AGMR, Moreira A, Costa Jr. JR, Maldonado G, Cano $M$, et al. Trombose de stent farmacológico no "mundo-real": análise crítica do Registro DESIRE (Drug-Eluting Stent in the Real World). Revista Brasileira de Cardiologia Invasiva. 2008;16:144-54.

61. Costa Jr. JdR, Sousa AGMR, Costa R, Moreira A, Cano M, Maldonado G, et al. Até que ponto a complexidade angiográfica influi nos resultados tardios de pacientes tratados com stents farmacológicos?: comparação entre indicações on-label e off-label no Registro DESIRE. Revista Brasileira de Cardiologia Invasiva. 2009;17:31-8.

62.Costa Jr. JdR, Sousa AGMR, Moreira A, Costa R, Maldonado G, Cano M, et al. Intervenção coronária percutânea em pontes de veia safena com uso de stents farmacológicos: resultados agudos e tardios dos pacientes incluídos no registro DESIRE. Revista Brasileira de Cardiologia Invasiva. 2009;17:202-8.

63.Nogueira EF, Sousa AGMR, Costa Jr. JR, Moreira A, Costa R, Maldonado $G$, et al. Stents farmacológicos para o tratamento de coronárias de fino calibre: experiência muito tardia (até 7 anos) do Registro DESIRE. Revista Brasileira de Cardiologia Invasiva. 2010;18:288-93.

64. Tavares S, Sousa AGMR, Costa RA, Moreira A, Costa Jr. JR, Maldonado $\mathrm{G}$, et al. Impacto de stents farmacológicos em pacientes com doença arterial coronária estável submetidos a intervenção coronária percutânea na prática diária do mundo real. Revista Brasileira de Cardiologia Invasiva. 2010;18:392-9.

65.Cavalcante LP, Sousa AGMR, Costa RA, Moreira A, Costa Jr. JR, Maldonado G, et al. Incidência e preditores de revascularização da lesão- 
alvo no seguimento clínico de longo prazo: análise crítica do Registro DESIRE. Revista Brasileira de Cardiologia Invasiva. 2010;18:157-64.

66. Collet CA, Costa Jr. JR, Sousa AGMR, Feres F, Moreira A, Costa R, et al. Stents farmacológicos vs. stents não-farmacológicos no tratamento de enxertos de veia safena. Revista Brasileira de Cardiologia Invasiva. 2011;19:122-30.

67.Costa RA, Sousa AGMR, Costa Jr. JR, Moreira A, Maldonado G, Cano $M N$, et al. Evolução tardia de pacientes com infarto agudo do miocárdio tratados com stents farmacológicos na prática diária do mundo real: subanálise do Registro DESIRE (Drug-Eluting Stent In the REal World). Revista Brasileira de Cardiologia Invasiva. 2011;19:244-54.

68.Bernardi BP, Sousa AGMR, Costa Jr. JR, Moreira A, Costa RAd, Cano M, et al. Avaliação muito tardia do uso de stents farmacológicos para tratamento de pacientes com lesões em enxertos de veia safena: experiência de uma década do Registro DESIRE. Revista Brasileira de Cardiologia Invasiva. 2013;21:115-20.

69.Venkitachalam L, Kip KE, Selzer F, Wilensky RL, Slater J, Mulukutla SR, et al. Twenty-year evolution of percutaneous coronary intervention and its impact on clinical outcomes: a report from the National Heart, Lung, and Blood Institute-sponsored, multicenter 1985-1986 PTCA and 1997-2006 Dynamic Registries. Circ Cardiovasc Interv. 2009;2(1):6-13.

70.De Luca G, Suryapranata H, van 't Hof AW, de Boer MJ, Hoorntje JC, Dambrink JH, et al. Prognostic assessment of patients with acute myocardial infarction treated with primary angioplasty: implications for early discharge. Circulation. 2004;109(22):2737-43. 
71.Kimmel SE, Berlin JA, Strom BL, Laskey WK. Development and validation of simplified predictive index for major complications in contemporary percutaneous transluminal coronary angioplasty practice. The Registry Committee of the Society for Cardiac Angiography and Interventions. J Am Coll Cardiol. 1995;26(4):931-8.

72.de Belder AJ, Jewitt DE, Wainwright RJ, Thomas MR. Development and validation of a Bayesian index for predicting major adverse cardiac events with percutaneous transluminal coronary angioplasty. Heart. 2001;85(1):6972.

73.Dash H, Johnson RA, Dinsmore RE, Harthorne JW. Cardiomyopathic syndrome due to coronary artery disease. I: Relation to angiographic extent of coronary disease and to remote myocardial infarction. $\mathrm{Br}$ Heart $\mathrm{J}$. 1977;39(7):733-9.

74.Califf RM, Phillips HR, 3rd, Hindman MC, Mark DB, Lee KL, Behar VS, et al. Prognostic value of a coronary artery jeopardy score. J Am Coll Cardiol. 1985;5(5):1055-63.

75.Coronary artery surgery study (CASS): a randomized trial of coronary artery bypass surgery. Quality of life in patients randomly assigned to treatment groups. Circulation. 1983;68(5):951-60.

76.Emond M, Mock MB, Davis KB, Fisher LD, Holmes DR, Jr., Chaitman BR, et al. Long-term survival of medically treated patients in the Coronary Artery Surgery Study (CASS) Registry. Circulation. 1994;90(6):2645-57.

77.Myers WO, Blackstone EH, Davis K, Foster ED, Kaiser GC. CASS Registry long term surgical survival. Coronary Artery Surgery Study. J Am Coll Cardiol. 1999;33(2):488-98. 
78.Ryan TJ, Faxon DP, Gunnar RM, Kennedy JW, King SB, 3rd, Loop FD, et al. Guidelines for percutaneous transluminal coronary angioplasty. A report of the American College of Cardiology/American Heart Association Task Force on Assessment of Diagnostic and Therapeutic Cardiovascular Procedures (Subcommittee on Percutaneous Transluminal Coronary Angioplasty). Circulation. 1988;78(2):486-502.

79.Ellis SG, Vandormael MG, Cowley MJ, DiSciascio G, Deligonul U, Topol EJ, et al. Coronary morphologic and clinical determinants of procedural outcome with angioplasty for multivessel coronary disease. Implications for patient selection. Multivessel Angioplasty Prognosis Study Group. Circulation. 1990;82(4):1193-202.

80.Krone RJ, Shaw RE, Klein LW, Block PC, Anderson HV, Weintraub WS, et al. Evaluation of the American College of Cardiology/American Heart Association and the Society for Coronary Angiography and Interventions lesion classification system in the current "stent era" of coronary interventions (from the ACC-National Cardiovascular Data Registry). Am J Cardiol. 2003;92(4):389-94.

81.Smith SC, Jr., Dove JT, Jacobs AK, Kennedy JW, Kereiakes D, Kern MJ, et al. ACC/AHA guidelines of percutaneous coronary interventions (revision of the 1993 PTCA guidelines)--executive summary. A report of the American College of Cardiology/American Heart Association Task Force on Practice Guidelines (committee to revise the 1993 guidelines for percutaneous transluminal coronary angioplasty). J Am Coll Cardiol. 2001;37(8):2215-39.

82.Nashef SA, Roques F, Michel P, Gauducheau E, Lemeshow S, Salamon R. European system for cardiac operative risk evaluation (EuroSCORE). Eur J Cardiothorac Surg. 1999;16(1):9-13. 
83.Ranucci M, Castelvecchio S, Menicanti L, Frigiola A, Pelissero G. Risk of assessing mortality risk in elective cardiac operations: age, creatinine, ejection fraction, and the law of parsimony. Circulation. 2009;119(24):305361.

84.Sianos G, Morel MA, Kappetein AP, Morice MC, Colombo A, Dawkins K, et al. The SYNTAX Score: an angiographic tool grading the complexity of coronary artery disease. Eurolntervention. 2005;1(2):219-27.

85.Capodanno D, Miano M, Cincotta G, Caggegi A, Ruperto C, Bucalo R, et al. EuroSCORE refines the predictive ability of SYNTAX score in patients undergoing left main percutaneous coronary intervention. Am Heart J. 2010;159(1):103-9.

86.Garg S, Sarno G, Garcia-Garcia HM, Girasis C, Wykrzykowska J, Dawkins KD, et al. A new tool for the risk stratification of patients with complex coronary artery disease: the Clinical SYNTAX Score. Circ Cardiovasc Interv. 2010;3(4):317-26.

87.Farooq V, Girasis C, Magro M, Onuma Y, Morel MA, Heo JH, et al. The CABG SYNTAX Score - an angiographic tool to grade the complexity of coronary disease following coronary artery bypass graft surgery: from the SYNTAX Left Main Angiographic (SYNTAX-LE MANS) substudy. Eurolntervention. 2013;8(11):1277-85.

88.Singh M, Rihal CS, Lennon RJ, Spertus J, Rumsfeld JS, Holmes DR, Jr. Bedside estimation of risk from percutaneous coronary intervention: the new Mayo Clinic risk scores. Mayo Clin Proc. 2007;82(6):701-8.

89.Singh M, Peterson ED, Milford-Beland S, Rumsfeld JS, Spertus JA. Validation of the Mayo clinic risk score for in-hospital mortality after 
percutaneous coronary interventions using the national cardiovascular data registry. Circ Cardiovasc Interv. 2008;1(1):36-44.

90.Singh M, Gersh BJ, Li S, Rumsfeld JS, Spertus JA, O'Brien SM, et al. Mayo Clinic Risk Score for percutaneous coronary intervention predicts inhospital mortality in patients undergoing coronary artery bypass graft surgery. Circulation. 2008;117(3):356-62.

91.Romagnoli E, Burzotta F, Trani C, Siviglia M, Biondi-Zoccai GG, Niccoli $\mathrm{G}$, et al. EuroSCORE as predictor of in-hospital mortality after percutaneous coronary intervention. Heart. 2009;95(1):43-8.

92.Chowdhary S, Ivanov J, Mackie K, Seidelin PH, Dzavik V. The Toronto score for in-hospital mortality after percutaneous coronary interventions. Am Heart J. 2009;157(1):156-63.

93. Hamburger JN, Walsh SJ, Khurana R, Ding L, Gao M, Humphries KH, et al. Percutaneous coronary intervention and 30-day mortality: the British Columbia PCI risk score. Catheter Cardiovasc Interv. 2009;74(3):377-85.

94.Peterson ED, Dai D, DeLong ER, Brennan JM, Singh M, Rao SV, et al. Contemporary mortality risk prediction for percutaneous coronary intervention: results from 588,398 procedures in the National Cardiovascular Data Registry. J Am Coll Cardiol. 2010;55(18):1923-32.

95.Kereiakes DJ. Cultivating prognosis following percutaneous coronary intervention: the American College of Cardiology/National Cardiovascular Data Registry risk score. J Am Coll Cardiol. 2010;55(18):1933-5.

96. Hannan EL, Farrell LS, Walford G, Jacobs AK, Berger PB, Holmes DR, Jr., et al. The New York State risk score for predicting in-hospital/30-day 
mortality following percutaneous coronary intervention. JACC Cardiovasc Interv. 2013;6(6):614-22.

97.Farooq V, van Klaveren D, Steyerberg EW, Meliga E, Vergouwe Y, Chieffo $A$, et al. Anatomical and clinical characteristics to guide decision making between coronary artery bypass surgery and percutaneous coronary intervention for individual patients: development and validation of SYNTAX score II. Lancet. 2013;381(9867):639-50.

98.Kovacic JC, Limaye AM, Sartori S, Lee P, Patel R, Chandela S, et al. Comparison of six risk scores in patients with triple vessel coronary artery disease undergoing $\mathrm{PCl}$ : competing factors influence mortality, myocardial infarction, and target lesion revascularization. Catheter Cardiovasc Interv. 2013;82(6):855-68.

99.Wu C, Hannan EL, Walford G, Ambrose JA, Holmes DR, Jr., King SB, 3rd, et al. A risk score to predict in-hospital mortality for percutaneous coronary interventions. J Am Coll Cardiol. 2006;47(3):654-60.

100.Sousa J E M R SA, Costa Jr JR, Costa R, Moreira A, Neto CMC, Cano M, Maldonado G. Registro DESIRE: Drug-eluting stents in the real world. In: Sousa JEMR SA, editor. REGISTRO DESIRE:Uma década (2002-2012) de emprego clinico dos stents farmacológicos no tratamento da Doença Arterial Coronária. São Paulo: Ed Atheneu; 2012. p. 3-31.

101.Tanajura LFL. Preparo do paciente para intervenção coronária percutânea. 2 ed. São Paulo: Editora Atheneu; 2014. 329-33 p.

102.Costa R PW, Sousa JEMR. Angiografia Coronária Quantitativa: Técnica e Mensurações. In: J. Eduardo M. R. Sousa AGMRS, editor. registro DESIRE: uma década (2002-2012) de emprego clínico dos stents 
farmacológicos no tratamento da Doença Arterial Coronária. São Paulo: Editora Atheneu; 2012. p. 177-84.

103.Alderman EL, Stadius M. The angiographie definitions of the Bypass Angioplasty Revascularization Investigation. Coronary Artery Disease. 1992;3(12):1189-208.

104. Medina A, Suarez de Lezo J, Pan M. [A new classification of coronary bifurcation lesions]. Rev Esp Cardiol. 2006;59(2):183.

105. Mehran R, Dangas G, Abizaid AS, Mintz GS, Lansky AJ, Satler LF, et al. Angiographic patterns of in-stent restenosis: classification and implications for long-term outcome. Circulation. 1999;100(18):1872-8.

106. Neto CdMC. Apresentação do Sistema e dos Métodos de Informática do Registro DESIRE. In: J. Eduardo M. R. Sousa AGMRS, editor. Registro DESIRE: Uma década (2002-2012) de emprego clínico dos stents farmacológicos no tratamento da Doença Arterial Coronária. São Paulo: Editora Atheneu; 2012. p. 163-74.

107.Campeau L. Letter: Grading of angina pectoris. Circulation. 1976;54(3):522-3.

108.Braunwald E. Unstable angina. A classification. Circulation. 1989;80(2):410-4.

109. Lim CC, van Gaal WJ, Testa L, Cuculi F, Arnold JR, Karamitsos T, et al. With the "universal definition," measurement of creatine kinase-myocardial band rather than troponin allows more accurate diagnosis of periprocedural necrosis and infarction after coronary intervention. J Am Coll Cardiol. 2011;57(6):653-61. 
110.Cutlip DE, Windecker S, Mehran R, Boam A, Cohen DJ, van Es GA, et al. Clinical end points in coronary stent trials: a case for standardized definitions. Circulation. 2007;115(17):2344-51.

111.R: A Language and enviroment for statistical computing. [Internet]. $R$ Foundation for Statistical Computing. 2016. Available from: https://www.Rproject.org/.

112. Colosimo E A GSR. Modelo Semi-Paramétrico de Riscos Proporcionais de Cox. ANÁLISE DE SOBREVIVÊNCIA APLICADA: Blucher; 2006. p. 11143.

113.Venable WN RB. An Unbalanced Four-Way Layout, subseção Automated modelo selection. Modern Applied Statistics with S. 4th ed: Springer; 2002. p. 175-7.

114. Heagerty PJ, Zheng Y. Survival Model Predictive Accuracy and ROC Curves. Biometrics. 2005;61(1):92-105.

115.Palmerini T, Benedetto U, Biondi-Zoccai G, Della Riva D, BacchiReggiani L, Smits PC, et al. Long-Term Safety of Drug-Eluting and BareMetal Stents: Evidence From a Comprehensive Network Meta-Analysis. J Am Coll Cardiol. 2015;65(23):2496-507.

116.Navarese EP, Kowalewski M, Kandzari D, Lansky A, Gorny B, Koltowski $\mathrm{L}$, et al. First-generation versus second-generation drug-eluting stents in current clinical practice: updated evidence from a comprehensive metaanalysis of randomised clinical trials comprising 31379 patients. Open Heart. 2014;1(1):e000064. 
117.Wu C, Camacho FT, King SB, 3rd, Walford G, Holmes DR, Jr., Stamato NJ, et al. Risk stratification for long-term mortality after percutaneous coronary intervention. Circ Cardiovasc Interv. 2014;7(1):80-7.

118.Kassaian SE, Saroukhani S, Alaeddini F, Salarifar M, Capodanno D, Poorhoseini $\mathrm{H}$, et al. A Risk-Scoring Model to Predict One-year Major Adverse Cardiac Events after Percutaneous Coronary Intervention. J Tehran Heart Cent. 2015;10(4):167-75.

119.Abdelmeguid AE, Topol EJ, Whitlow PL, Sapp SK, Ellis SG. Significance of mild transient release of creatine kinase-MB fraction after percutaneous coronary interventions. Circulation. 1996;94(7):1528-36.

120.Abdelmeguid AE, Topol EJ. The myth of the myocardial 'infarctlet' during percutaneous coronary revascularization procedures. Circulation. 1996;94(12):3369-75.

121. Thygesen K, Alpert JS, White HD, Joint ESCAAHAWHFTFftRoMI, Jaffe AS, Apple FS, et al. Universal definition of myocardial infarction. Circulation. 2007;116(22):2634-53.

122. Thygesen K, Alpert JS, Jaffe AS, Simoons ML, Chaitman BR, White HD, et al. Third universal definition of myocardial infarction. Circulation. 2012;126(16):2020-35.

123.Ndrepepa G, Colleran R, Braun S, Cassese S, Hieber J, Fusaro M, et al. High-Sensitivity Troponin $\mathrm{T}$ and Mortality After Elective Percutaneous Coronary Intervention. J Am Coll Cardiol. 2016;68(21):2259-68.

124.Ndrepepa G, Braun S, Cassese S, Mayer K, Lohaus R, Lahmann AL, et al. Prognostic Value of High-sensitivity Troponin $\mathrm{T}$ After Percutaneous 
Coronary Intervention in Patients With Stable Coronary Artery Disease. Rev Esp Cardiol (Engl Ed). 2016;69(8):746-53.

125. Weintraub WS, Grau-Sepulveda MV, Weiss JM, Delong ER, Peterson ED, O'Brien SM, et al. Prediction of long-term mortality after percutaneous coronary intervention in older adults: results from the National Cardiovascular Data Registry. Circulation. 2012;125(12):1501-10.

126. MacKenzie TA, Malenka DJ, Olmstead EM, Piper WD, Langner C, Ross $\mathrm{CS}$, et al. Prediction of survival after coronary revascularization: modeling short-term, mid-term, and long-term survival. Ann Thorac Surg. 2009;87(2):463-72.

127.Grayson AD, Moore RK, Jackson M, Rathore S, Sastry S, Gray TP, et al. Multivariate prediction of major adverse cardiac events after 9914 percutaneous coronary interventions in the north west of England. Heart. 2006;92(5):658-63.

128.Serruys PW, Morice MC, Kappetein AP, Colombo A, Holmes DR, Mack $\mathrm{MJ}$, et al. Percutaneous coronary intervention versus coronary-artery bypass grafting for severe coronary artery disease. N Engl J Med. 2009;360(10):96172.

* De acordo com:

Adaptado de International Committee of Medical Journals Editors (Vancouver). Universidade de São Paulo. Faculdade de Medicina. Serviço de Biblioteca e Documentação. Guia de apresentação de dissertações, 
teses e monografias da FMUSP. Elaborado por Anneliese Carneiro da Cunha, Maria Julia A.L. Freddi, Maria F. Crestana, Marinalva de S. Aragão, Suely C. Cardoso, Valéria Vilhena. 3a ed. São Paulo: Divisão de Biblioteca e Documentação; 2010. Abreviaturas dos títulos dos periódicos de acordo com List of Journals Indexed in Index Medicus. 